\title{
SUR CERTAINS GROUPES D'OPÉRATEURS UNITAIRES
}

\author{
PAR
}

\author{
ANDRE WEIL \\ Institute for Advanced Study, Princeton, N.J., U.S.A. (1)
}

A force d'habitude, le fait que les séries thêta définissent des fonctions modulaires a presque cessé de nous étonner. Mais l'apparition du groupe symplectique comme un deus ex machina dans les célèbres travaux de Siegel sur les formes quadratiques n'a rien perdu encore de son caractère mystérieux. Le but de ce mémoire, et de ceux qui lui feront suite, n'est pas, bien entendu, d'élucider définitivement la question, mais de jeter un peu de lumière sur certains aspects de cette théorie qui étaient restés dans l'ombre jusqu'à présent.

Une analyse attentive des travaux de Siegel permet en effet de déceler le rôle capital qu'y joue une certaine représentation unitaire, non pas du groupe symplectique lui-même, mais d'une extension centrale de ce groupe, ainsi que des groupes analogues sur les corps $p$-adiques. Il se trouve que cette représentation, pour le cas du groupe symplectique réel (et même de sa généralisation naturelle à l'espace de Hilbert), a été définie et étudiée récemment par D. Shale [7], après que son existence, ou du moins celle de la représentation projective correspondante, eût été reconnue par I. Segal [5] à propos de mécanique quantique. Celui-ci est revenu sur la question [6], à la suite d'un exposé où j'en avais souligné l'importance en théorie des nombres; j'ai emprunté à son travail, dont je lui suis reconnaissant de m'avoir communiqué le manuscrit, le principe de la démonstration du théorème d'existence (théorème 1 ci-dessous; pour une autre démonstration, ef. G. Mackey [3]), ainsi que l'idée de prendre pour point de départ la théorie générale des groupes abéliens localement compacts.

En conséquence, les théorèmes fondamentaux concernant la représentation unitaire en question seront exposés au Chapitre I pour un groupe abélien localement compact qui n'est soumis à aucune hypothèse restrictive; qu'il me soit permis, en passant, de signaler l'intérêt qu'il y aurait peut-être à examiner de plus près, du point de vue de la

(1) Pendant la période où ce mémoire a été rédigé, j'ai été supporté à la fois par la National Science Foundation (contrat GP-823) et par l'Institut des Hautes Etudes Scientifiques de Bures-surYvette; à l'une et à l'autre je suis heureux d'exprimer ici ma reconnaissance.

10-642946 Acta mathematica. 111. Imprimé le 3 juin 1964. 
présente théorie, le cas des groupes finis $\left.{ }^{(}\right)$. Le Chapitre II fixe les notations en vue de l'application des résultats qui précèdent aux espaces vectoriels sur les corps locaux (c'està-dire les corps localement compacts non discrets) et sur les anneaux adéliques; et il fait servir les théorèmes 2 et 5 du Chapitre $I$ à une démonstration de la loi de réciprocité quadratique, apparentée à celle qui figure au dernier chapitre du livre classique de Hecke sur les corps de nombres algébriques. Une démonstration directe très simple des théorèmes 2 et 5 , indépendante de la théorie exposée au Chapitre I, a été obtenue récemment par $P$. Cartier (cf. [2]); jointe aux considérations du Chapitre II du présent mémoire, et notamment aux propositions 3 et 4 de celui-ci, elle constitue à certains égards la méthode la plus satisfaisante qui soit actuellement connue pour établir la loi de réciprocité quadratique sous sa forme la plus générale. Le Chapitre III spécialise au "cas local " et au " cas adélique " la théorie du Chapitre I, en traitant en détail de questions de continuité qu'il n'était guère possible d'aborder utilement dans le cadre de celui-ci. C'est ainsi qu'on obtient, dans le cas local et dans le cas adélique, une représentation unitaire d'un groupe localement compact (dit " métaplectique ") qui, sauf en caractéristique 2, est une extension centrale du groupe symplectique par le tore $T$. Au Chapitre IV, on fait voir que celle-ci peut se réduire à une extension, en général non triviale, du même groupe par le groupe $\{ \pm 1\}$; autrement dit, la classe de cohomologie qui la détermine est toujours d'ordre 2 et n'est pas nulle en général; bien que ce résultat ne doive nous être d'aucune utilité par la suite, il répond à une question si naturelle qu'il n'a pas semblé superflu de l'insérer ici. Enfin le Chapitre $\mathrm{V}$ fixe les notations en vue de la spécialisation des résultats ci-dessus au cas des algèbres à involution, indispensable pour les applications aux groupes classiques; il donne, en vue de ces applications, quelques résultats auxiliaires, et il se termine sur l'énoncé d'une formule qui généralise des résultats classiques de Siegel et dont la démonstration formera l'objet principal du mémoire suivant.

Table des notations

\begin{tabular}{|c|c|}
\hline 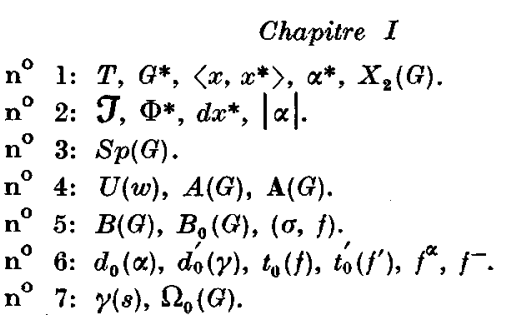 & 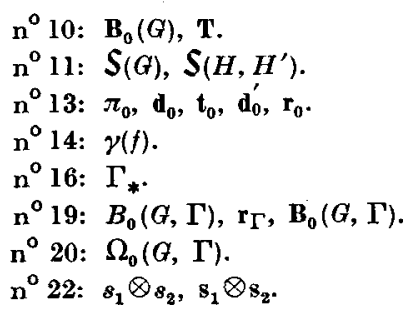 \\
\hline
\end{tabular}

(1) Cette question semble apparentée, aux problèmes étudiés par H. D. Kloosterman dans: The behaviour of general theta functions under the modular group and the characters of binary modular congruence groups, Ann. of Math., 47 (1946), pp. 317-447. 
Chapitre II

$\mathrm{n}^{\circ} 23: X^{*},\left[x, x^{*}\right], \alpha^{*}, Q(X), Q_{a}(X)$.

$\mathrm{n}^{\circ} 24: \mathrm{v}, \mathfrak{p}, \chi, \gamma(f)$.

$\mathrm{n}^{0} 26: q_{m}$.

n $27: L_{*}$.

$\mathrm{n}^{\circ} 29: k_{v}, \mathrm{o}_{v}, A_{k}, X_{k}, X_{A}, X_{v}, X_{v}^{\circ}, S, X_{S}^{\circ}, \chi, \chi_{v}$

$\mathrm{n}^{\mathrm{o}}$ 30: $\gamma_{v}(f), \gamma(f)$.

Chapitre III

$\mathrm{n}^{\circ}$ 31: $B\left(z_{1}, z_{2}\right), S p(X), \mathfrak{U}(X),(\sigma, f), P s(X)$.

$\mathrm{n}^{\circ}$ 32: Aut $(X), d(\alpha)$, Is $\left(X^{*}, X\right), d^{\prime}(\gamma), t(f), t^{\prime}\left(f^{\prime}\right)$, $\mathrm{n}^{\circ} 33: \mu$. $\Omega(X), P s^{+}(X), P s^{-}(X)$

$\mathbf{n}^{0}$ 34: $M p(X), \pi, \mathbf{T}, \mathbf{d}(\alpha), \mathbf{d}^{\prime}(\gamma), \mathbf{t}(f), \mathbf{t}^{\prime}\left(f^{\prime}\right), \mathbf{r}(s)$.

$\mathbf{n}^{\circ}$ 36: $P s(X, L), \mathbf{r}_{L}, \mathbf{r}_{L}^{\prime}$. $\mathrm{n}^{\circ}$ 37: $P_{s}(X)_{k}, P s(X)_{v}, P s(X)_{A}, P s(X)_{v}^{\circ}, P s(X)_{S}^{\circ}$, $S_{\infty}, \mu_{A}, M p(X)_{A}, \pi, \mathbf{T}$.

$\mathrm{n}^{0}$ 38: $\Omega_{v}, \Omega_{S}, \mathbf{r}_{v}^{\prime}, \mathbf{r}_{S}$.

$\mathrm{n}^{\circ} 40: \mathbf{r}_{k}$.

$$
\text { Chapitre IV }
$$

$\mathrm{n}^{\circ} 43: S p_{1}(X), S p_{2}(X), M p^{+}(X), P s_{2}^{+}(X)$.

$\mathrm{n}^{\circ} 45: \pi_{v}, M p(X) \stackrel{\circ}{*}, M(S), M p(X) \stackrel{\circ}{S}$.

Chapitre $V$

$\mathrm{n}^{\circ}$ 46: $P(\mathrm{X})$.

$\mathrm{n}^{\circ} 49: \mathcal{A}, \iota, \tau, X^{*},\left\{x, x^{*}\right\}, \alpha^{*}, Q(X / \mathcal{A}), Q_{a}(X / \mathcal{A})$, Aut $(X / \mathcal{A}), \operatorname{Is}(X, Y / \mathcal{A}), P s(X / k), P s(X / \mathcal{A})$.

$\mathrm{n}^{0} 5 \mathrm{l}: \quad P(X / k), \quad P(X / \mathcal{A}), \quad M p(X / k), \quad M p(X / \mathcal{A})$, $M p(X / k)_{A}, M p(X / \mathcal{A})_{A}$.

\section{Groupes abéliens localement compacts}

1. Dans ce chapitre, il s'agira principalement d'un groupe abélien localement compact $G$, sur lequel on ne fera le plus souvent aucune hypothèse restrictive; mais une partie de nos résultats est sans intérêt à moins que $G$ ne soit isomorphe à son dual. Toutes les applications ultérieures se rapporteront à l'un des cas suivants: $(a) G$ est un espace vectoriel $X$ de dimension finie sur un corps $k$ localement compact non discret; $(b) G$ est de la forme $X_{A}=X_{k} \otimes A_{k}$, où $A_{k}$ est l'anneau des adèles d'un corps $k$ qui peut être, soit un corps de nombres algébriques, soit un corps de fonctions algébriques de dimension 1 sur un corps fini, et où $X_{k}$ est un espace vectoriel de dimension finie sur $k$. On se référera à ces cas en disant que $G$ est "de type local " dans le cas (a), "de type adélique " dans le cas (b); si $G$ est de type local ou adélique, il est isomorphe à son dual. Les groupes abéliens localement compacts seront le plus souvent notés additivement.

On désignera par $T$ le groupe multiplicatif des nombres complexes $t$ tels que $t \bar{t}=1$; un caractère de $G$ est donc un morphisme de $G$ dans $T$. Si $G$ et $H$ sont des groupes abéliens localement compacts, un bicaractère de $G \times H$ sera une application continue $f$ de $G \times H$ dans $T$ telle que, pour tout $y \in H, x \rightarrow f(x, y)$ soit un caractère de $G$ et que, pour tout $x \in G, y \rightarrow f(x, y)$ soit un caractère de $H$.

Une application continue $f$ de $G$ dans $T$ sera appelée un caractère du second degré de $G$ si l'application

$$
(x, y) \rightarrow f(x+y) f(x)^{-1} f(y)^{-1}
$$

est un bicaractère de $G \times G$, ou, ce qui revient au même, si $f$ satisfait à la relation

$$
f(x+y+z) f(x) f(y) f(z)=f(x+y) f(y+z) f(z+x)
$$

quels que soient $x, y, z$ dans $G$. 
On notera toujours $G^{*}$ le dual de $G$ (noté additivement, lui aussi), et on notera $\left\langle x, x^{*}\right\rangle$, pour $x \in G, x^{*} \in G^{*}$, la valeur en $x$ du caractère de $G$ qui correspond à $x^{*}$. On conviendra une fois pour toutes d'identifier avec $G$ le bidual $\left(G^{*}\right)^{*}$ de $G$ de telle sorte que l'on ait

$$
\left\langle x, x^{*}\right\rangle=\left\langle x^{*}, x\right\rangle
$$

(on pourrait tout aussi bien convenir de faire cette identification de manière à avoir $\left\langle x, x^{*}\right\rangle=\left\langle-x^{*}, x\right\rangle$, et ce serait même plus commode à certains égards, mais cela choquerait trop d'habitudes reçues). Si $x \rightarrow x \alpha$ est un morphisme de $G$ dans $H$, son $d u a l \alpha^{*}$ sera le morphisme de $H^{*}$ dans $G^{*}$ tel que l'on ait

$$
\left\langle x \alpha, y^{*}\right\rangle=\left\langle x, y^{*} \alpha^{*}\right\rangle
$$

quels que soient $x \in G, y^{*} \in H^{*}$. Tout bicaractère de $G \times H$ s'écrit d'une manière et d'une seule sous la forme

$$
f(x, y)=\langle x, y \alpha\rangle=\left\langle y, x \alpha^{*}\right\rangle
$$

où $\alpha$ est un morphisme de $H$ dans $G^{*}, \alpha^{*}$ étant done le morphisme de $G$ dans $H^{*}$ dual de $\alpha$. Si $G=H$, il faut et il suffit, pour que $f$ soit symétrique en $x, y$, que l'on ait $\alpha=\alpha^{*}$; on dira alors que le morphisme $\alpha$ de $G$ dans $G^{*}$ est symétrique.

Si $f$ est un caractère du second degré de $G$, on aura

$$
f(x+y) f(x)^{-1} f(y)^{-1}=\langle x, y \varrho\rangle
$$

où $\varrho=\varrho(f)$ est un morphisme, évidemment symétrique, de $G$ dans $G^{*}$; on exprimera (1) en disant que $f$ et $\varrho$ sont associés l'un à l'autre. Si on désigne par $X_{2}(G)$ le groupe multiplicatif des caractères du second degré de $G$, l'application $f \rightarrow \varrho(f)$ est un homomorphisme de $X_{2}(G)$ dans le groupe additif des morphismes symétriques de $G$ dans $G^{*}$; le noyau de cet homomorphisme est le groupe multiplicatif $X_{1}(G)$ des caractères de $G$. On peut en dire plus dans le cas où $x \rightarrow 2 x$ est un automorphisme de $G$ (ce qui a lieu par exemple si $G$ est de type local ou adélique sur un corps $k$ de caractéristique autre que 2); lorsqu'il en est ainsi, on notera $x \rightarrow 2^{-1} x$ l'automorphisme de $G$ inverse de $x \rightarrow 2 x$. En ce cas, si $\varrho$ est un morphisme symétrique de $G$ dans $G^{*}$, il est associé au caractère du second degré $f_{\varrho}(x)=$ $\left\langle x, 2^{-1} x \varrho\right\rangle$; si on note $X_{2}^{\circ}(G)$ le sous-groupe de $X_{2}(G)$ formé par les $f_{\varrho}$, on a alors $X_{2}(G)=$ $X_{2}^{\circ}(G) \times X_{1}(G)$, et $X_{2}^{\circ}(G)$ est isomorphe au groupe additif des morphismes symétriques de $G$ dans $G^{*}$.

On dira que le caractère du second degré f est non dégénéré si le morphisme symétrique $\varrho$ associé à $f$ est un isomorphisme de $G$ sur $G^{*}$; pour qu'il existe de tels caractères, il est nécessaire (mais non suffisant) que $G$ soit isomorphe à $G^{*}$. 
2. Une mesure de Haar $d x$ étant choisie dans $G$, la transformation de Fourier $\mathcal{T}$, relative à ce choix, est celle qui, à une fonction $\Phi$ sur $G$, associe la fonction $\Phi^{*}=\mathcal{T}(\Phi)$ sur $G^{*}$ définie par

$$
\Phi^{*}\left(x^{*}\right)=\int \Phi(x) \cdot\left\langle x, x^{*}\right\rangle \cdot d x
$$

lorsque cette intégrale a un sens, et par un prolongement convenable dans d'autres cas. Il y a alors, sur $G^{*}$, une mesure de Haar $d x^{*}$ et une seule, dite la duale de $d x$, telle que la transformation $\mathcal{J}^{-1}$ inverse de $\mathcal{J}$ soit donnée par la formule

$$
\Phi(x)=\int \Phi^{*}\left(x^{*}\right) \cdot\left\langle x,-x^{*}\right\rangle \cdot d x^{*}
$$

pour cette mesure, on a la formule de Plancherel

$$
\int|\Phi(x)|^{2} d x=\int\left|\Phi^{*}\left(x^{*}\right)\right|^{2} d x^{*}
$$

Il est clair que, pour tout $c>0$, la mesure de Haar sur $G^{*}$, duale de $c \cdot d x$, est $c^{-1} d x^{*}$. Cette remarque peut encore s'exprimer comme suit:

Lемм 1. Soient $G, H$ deux groupes abéliens localement compacts, munis de mesures de Haar $d x$, dy; soient $G^{*}, H^{*}$ leurs duaux, munis des mesures de Haar $d x^{*}, d y^{*}$ duales de $d x$ et de dy. Alors, si $\alpha$ est un isomorphisme de $G$ sur $H, \alpha^{*}$ est un isomorphisme de $H^{*}$ sur $G^{*}$, et on $a\left|\alpha^{*}\right|=|\alpha|$.

Rappelons que, si $G$ et $H$ sont des groupes localement compacts (commutatifs ou non) munis de mesures de Haar, le module d'un isomorphisme $\alpha$ de $G$ sur $H$ est le nombre $|\alpha|=d(x \alpha) / d x$ défini par la formule

$$
\int F(y) d y=|\alpha| \cdot \int F(x \alpha) d x
$$

où $F \in L^{1}(H)$; si $G=H$, il est généralement sous-entendu qu'on prend $d x=d y$, et alors $|\alpha|$ est indépendant du choix de $d x$. Pour démontrer le lemme, posons $m=|\alpha|$; par transport de structure, $\alpha$ transforme $d x$ en une mesure de Haar $d^{\prime} y$ sur $H$, et on voit aussitôt que $d^{\prime} y=m^{-1} d y$; il s'ensuit que $\alpha^{*}$ transforme en $d x^{*}$ la mesure duale de $d^{\prime} y$, qui est, comme on l'a vu plus haut, $m \cdot d y^{*}$; donc $\alpha^{*}$ transforme $d y^{*}$ en $m^{-1} d x^{*}$, ce qui démontre le lemme.

3. Soit $z \rightarrow z \sigma$ un automorphisme de $G \times G^{*}$; si on pose $z=\left(x, x^{*}\right)$, on pourra aussi écrire $\sigma$ sous forme " matricielle »: 


$$
\left(x, x^{*}\right) \rightarrow\left(x, x^{*}\right) \cdot\left(\begin{array}{ll}
\alpha & \beta \\
\gamma & \delta
\end{array}\right)
$$

ce qui veut dire, bien entendn :

$$
\left(x, x^{*}\right) \rightarrow\left(x \alpha+x^{*} \gamma, x \beta+x^{*} \delta\right)
$$

ici $\alpha, \beta, \gamma, \delta$ désignent des morphismes de $G$ dans $G$, de $G$ dans $G^{*}$, de $G^{*}$ dans $G$ et de $G^{*}$ dans $G^{*}$, respectivement. On notera que le dual $\sigma^{*}$ de l'automorphisme $\sigma$ de $G \times G^{*}$ défini par ces formules est l'automorphisme

$$
\sigma^{*}=\left(\begin{array}{ll}
\alpha^{*} & \gamma^{*} \\
\beta^{*} & \delta^{*}
\end{array}\right)
$$

de $G^{*} \times G$. Soit $\eta$ l'isomorphisme $\left(\begin{array}{rr}0 & 1 \\ -1 & 0\end{array}\right)$ de $G \times G^{*}$ sur $G^{*} \times G$, ou, ce qui revient au même, l'isomorphisme $\left(x, x^{*}\right) \rightarrow\left(-x^{*}, x\right)$ (nous désignerons souvent par 1 l'automorphisme identique d'un groupe, quel que soit ce groupe). La formule

$$
\sigma^{\mathrm{I}}=\eta \sigma^{*} \eta^{-1}=\left(\begin{array}{rr}
\delta^{*} & -\beta^{*} \\
-\gamma^{*} & \alpha^{*}
\end{array}\right)
$$

définit alors un automorphisme $\sigma^{\mathrm{I}} \operatorname{de} G \times G^{*}$, et le lemme 1 du $\mathrm{n}^{\circ} 2$ montre qu'on a $\left|\sigma^{\mathrm{I}}\right|=|\sigma|$. On notera que $\sigma \rightarrow \sigma^{\mathrm{I}}$ est un anti-automorphisme involutif du groupe des automorphismes de $G \times G^{*}$.

Pour la commodité de l'écriture, nous conviendrons de désigner par $F$ le bicaractère de $\left(G \times G^{*}\right) \times\left(G \times G^{*}\right)$ défini par

$$
F\left(z_{1}, z_{2}\right)=\left\langle x_{1}, x_{2}^{*}\right\rangle \quad\left(z_{1}=\left(x_{1}, x_{1}^{*}\right), z_{2}=\left(x_{2}, x_{2}^{*}\right)\right) .
$$

Un automorphisme $\sigma$ de $G \times G^{*}$ sera dit symplectique s'il laisse invariant le bicaractère $F\left(z_{1}, z_{2}\right) F\left(z_{2}, z_{1}\right)^{-1}$, c'est-à-dire si l'on a

$$
F\left(z_{1} \sigma, z_{2} \sigma\right) F\left(z_{2} \sigma, z_{1} \sigma\right)^{-1}=F\left(z_{1}, z_{2}\right) F\left(z_{2}, z_{1}\right)^{-1}
$$

quels que soient $z_{1}, z_{2}$ dans $G \times G^{*}$; on notera $S p(G)$ le groupe formé par ces automorphismes. Pour que $\sigma$ soit symplectique, il faut et il suffit (comme le montre un calcul immédiat) que $\sigma \sigma^{\mathbb{I}}=1, \sigma^{\mathrm{I}}$ étant défini par (2); comme on a $\left|\sigma^{\mathrm{I}}\right|=|\sigma|$, il s'ensuit que tout automorphisme symplectique est de module 1. La relation $\sigma \sigma^{I}=1$ donne en particulier $\alpha \beta^{*}=\beta \alpha^{*}$ et $\gamma \delta^{*}=\delta \gamma^{*}$, ce qui revient à dire que $\alpha \beta^{*}$ et $\gamma \delta^{*}$ sont des morphismes symétriques de $G$ dans $G^{*}$ et de $G^{*}$ dans $G$, respectivement; au moyen de la relation $\sigma^{\mathbf{I}} \sigma=1$, on voit qu'il en est de même $\operatorname{de} \beta^{*} \delta$ et $\gamma^{*} \alpha$. 
4. Pour tout élément $w=\left(u, u^{*}\right)$ de $G \times G^{*}$, on désignera par $U(w)$ l'opérateur qui, à toute fonction $\Phi$ sur $G$, associe la fonction $\Phi^{\prime}=U(w) \Phi$ donnée par

$$
\Phi^{\prime}(x)=(U(w) \Phi)(x)=\Phi(x+u) \cdot\left\langle x, u^{*}\right\rangle
$$

pour abréger, on écrira $U(w) \Phi(x)$ au lieu de $(U(w) \Phi)(x)$. Appliqués aux fonctions $\Phi \in L^{2}(G)$, les $U(w)$ sont évidemment des opérateurs unitaires, et on a, quels que soient $w_{1}, w_{2}$ dans $G \times G^{*}$ :

$$
U\left(w_{1}\right) U\left(w_{2}\right)=F\left(w_{1}, w_{2}\right) \cdot U\left(w_{1}+w_{2}\right)
$$

où $F$ est de nouveau la fonction définie par (3). On en conclut que les opérateurs $t \cdot U(w)$, pour $w \in G \times G^{*}$ et $t \in T$, forment un groupe, dont la loi de composition est donnée par

$$
\left(w_{1}, t_{1}\right) \cdot\left(w_{2}, t_{2}\right)=\left(w_{1}+w_{2}, F\left(w_{1}, w_{2}\right) t_{1} t_{2}\right)
$$

autrement dit, la formule (4) définit une loi de groupe sur l'ensemble $G \times G^{*} \times T$; et, si on désigne par $A(G)$ le groupe ainsi défini (qui, avec la topologie évidente sur $G \times G^{*} \times T$, est un groupe localement compact), l'application $(w, t) \rightarrow t \cdot U(w)$ définit une représentation unitaire de $A(G)$. On désignera par $\mathbf{A}(G)$ le groupe formé par les opérateurs $t \cdot U(w)$; si on le munit de la topologie induite par la topologie "forte " dans le groupe des automorphismes de $L^{2}(G)$ (cf. plus bas, $\mathrm{n}^{\circ} 35$ ), il est facile de vérifier que $(w, t) \rightarrow t \cdot U(w)$ est même un isomorphisme de groupes topologiques.

Le centre du groupe $A(G)$ est évidemment formé par les éléments $(0, t)$; il est isomorphe à $T$, et on le notera $T$ pour abréger. Il est clair que $(w, t) \rightarrow w$ est un homomorphisme de $A(G)$ sur $G \times G^{*}$, de noyau $T$; il permet d'identifier $A(G) / T$ à $G \times G^{*}$.

5. Soit $B(G)$ le groupe des automorphismes de $A(G)$. Un automorphisme $s$ de $B(G)$ induit, sur le centre $T$ de $A(G)$, un automorphisme, qui ne peut être que $t \rightarrow t$ ou $t \rightarrow \bar{t}$; et il induit, par passage au quotient, un automorphisme $\sigma$ sur $A(G) / T$, c'est-à-dire $G \times G^{*}$. On notera $B_{0}(G)$ le groupe des automorphismes de $A(G)$ qui induisent l'identité sur le centre $T$ de $A(G)$; c'est $B_{0}(G)$ que nous nous bornerons à considérer désormais, bien que les résultats qui suivent puissent en partie s'étendre à $B(G)$. Soit $s$ un élément de $B_{0}(G)$. induisant $\sigma$ sur $G \times G^{*}$; il est immédiat que $s$ peut s'écrire

$$
(w, t) s=(w \sigma, f(w) t),
$$

où $f$ est une application continue de $G \times G^{*}$ dans $T$. Pour que cette formule définisse un automorphisme de $A(G)$, il faut et il suffit que l'on ait

$$
f\left(w_{1}+w_{2}\right) f\left(w_{1}\right)^{-1} f\left(w_{2}\right)^{-1}=F\left(w_{1} \sigma, w_{2} \sigma\right) F\left(w_{1}, w_{2}\right)^{-1}
$$


quels que soient $w_{1}, w_{2}$ dans $G \times G^{*}$, ce qui montre en particulier que $f$ est un caractère du second degré de $G \times G^{*}$. De plus, en exprimant que le second membre est symétrique en $w_{1}$ et $w_{2}$, on voit que $\sigma$ doit être symplectique.

On écrira $s=(\sigma, f)$ quand $s$ est l'automorphisme de $A(G)$ défini par (5), $f$ et $\sigma$ satisfaisant à la relation (6). La loi de groupe dans $B_{0}(G)$ est alors donnée par

$$
(\sigma, f) \cdot\left(\sigma^{\prime}, f^{\prime}\right)=\left(\sigma \sigma^{\prime}, f^{\prime \prime}\right)
$$

où $f^{\prime \prime}$ est défini, pour tout $w \in G \times G^{*}$, par la formule

$$
f^{\prime \prime}(w)=f(w) f^{\prime}(w \sigma)
$$

L'application $s \rightarrow \sigma$ est un homomorphisme de $B_{0}(G)$ dans $S p(G)$; son noyau est formé par les éléments $(1, f)$, où $f$, d'après $(6)$, est un caractère de $G \times G^{*}$, donc de la forme

$$
f\left(u, u^{*}\right)=\left\langle u, a^{*}\right\rangle \cdot\left\langle a, u^{*}\right\rangle,
$$

avec $a \in G, a^{*} \in G^{*}$. Mais on vérifie immédiatement que $(1, f)$ est alors l'automorphisme intérieur de $A(G)$ déterminé par l'élément $\left(-a, a^{*}, 1\right)$. Le noyau de $s \rightarrow \sigma$ est donc formé par les automorphismes intérieurs de $A(G)$; il est isomorphe à $A(G) / T$, donc à $G \times G^{*}$.

On peut aller un peu plus loin en explicitant le second membre de (6); $\sigma$ étant mis sous forme matricielle comme au $n^{\circ} 3$, posons

$$
f^{\prime}\left(u, u^{*}\right)=f\left(u, u^{*}\right) \cdot\left\langle u^{*} \gamma,-u \beta\right\rangle
$$

un calcul facile permet alors de mettre (6) sous la forme

$$
f^{\prime}\left(u_{1}+u_{2}, u_{1}^{*}+u_{2}^{*}\right)=f^{\prime}\left(u_{1}, u_{1}^{*}\right) f^{\prime}\left(u_{2}, u_{2}^{*}\right) \cdot\left\langle u_{1}, u_{2} \alpha \beta^{*}\right\rangle \cdot\left\langle u_{1}^{*} \gamma \delta^{*}, u_{2}^{*}\right\rangle .
$$

Posons $g(u)=f^{\prime}(u, 0), h\left(u^{*}\right)=f^{\prime}\left(0, u^{*}\right)$; en faisant $u_{2}=0, u_{1}^{*}=0$ dans la relation ci-dessus, on voit que $f^{\prime}\left(u, u^{*}\right)$ n'est autre que $g(u) h\left(u^{*}\right)$, puis que $g$ et $h$ satisfont aux relations

$$
\begin{aligned}
& g\left(u_{1}+u_{2}\right)=g\left(u_{1}\right) g\left(u_{2}\right) \cdot\left\langle u_{1}, u_{2} \alpha \beta^{*}\right\rangle \\
& h\left(u_{1}^{*}+u_{2}^{*}\right)=h\left(u_{1}^{*}\right) h\left(u_{2}^{*}\right) \cdot\left\langle u_{1}^{*} \gamma \delta^{*}, u_{2}^{*}\right\rangle,
\end{aligned}
$$

ou autrement dit que ce sont des caractères du second degré de $G$ et de $G^{*}$, respectivement associés aux morphismes symétriques $\alpha \beta^{*}, \gamma \delta^{*} \operatorname{de} G$ dans $G^{*}$ et $\operatorname{de} G^{*} \operatorname{dans} G$. On a alors :

$$
f\left(u, u^{*}\right)=g(u) h\left(u^{*}\right) \cdot\left\langle u^{*} \gamma, u \beta\right\rangle \text {. }
$$

On a naturellement des résultats plus précis quand $x \rightarrow 2 x$ est un automorphisme de $G$. Compte tenu du $\mathrm{n}^{\circ} 1$, les formules ci-dessus montrent qu'alors, à tout automorphisme symplectique $\sigma$, il correspond un élément $(\sigma, f)$ de $B_{0}(G)$, qu'on obtient en prenant 


$$
g(u)=\left\langle u, 2^{-1} u \alpha \beta^{*}\right\rangle, \quad h\left(u^{*}\right)=\left\langle 2^{-1} u^{*} \gamma \delta^{*}, u^{*}\right\rangle .
$$

De plus, ees formules définissent un monomorphisme de $S p(G)$ dans $B_{0}(G)$, et $B_{0}(G)$ est le produit semidirect de l'image de $S p(G)$ par cette application et du groupe des automorphismes intérieurs de $A(G)$; par suite, $B_{0}(G)$ est alors isomorphe à un produit semidirect de $S p(G)$ par $G \times G^{*}$.

6. Revenant au cas général, soit toujours $s=(\sigma, f)$ un élément de $B_{0}(G)$, et écrivons $\sigma$ sous la forme matricielle introduite au $n^{\circ} 3$. Considérons d'abord le cas où $\beta=0$ et $\gamma=0$; la condition de symplecticité, $\sigma \sigma^{\mathbf{I}}=1$, donne alors $\delta=\alpha^{*-1}$, d'où résulte que le second membre de (6) a la valeur 1; on satisfait donc à (6) en prenant $f=1$, et $s$ ne diffère de $(\sigma, 1)$ que par un automorphisme intérieur. Pour tout automorphisme $\alpha$ de $G$, nous poserons

$$
d_{0}(\alpha)=\left(\left(\begin{array}{ll}
\alpha & 0 \\
0 & \alpha^{*-1}
\end{array}\right), 1\right)
$$

$\alpha \rightarrow d_{0}(\alpha)$ est donc un monomorphisme du groupe des automorphismes de $G$ dans le groupe $B_{0}(G)$.

Soient maintenant $\alpha=0, \delta=0$; comme $\sigma$ est un automorphisme de $G \times G^{*}$, cela implique que $\beta, \gamma$ sont des isomorphismes $\operatorname{de} G \operatorname{sur} G^{*}$ et $\operatorname{de} G^{*}$ sur $G$, respectivement; alors $\sigma \sigma^{\mathrm{I}}=1$ donne $\beta=-\gamma^{*-1}$, et on vérifie immédiatement qu'on satisfait à (6) en prenant $f\left(u, u^{*}\right)=$ $\left\langle u,-u^{*}\right\rangle$. Nous poserons, chaque fois que $\gamma$ est un isomorphisme de $G^{*} \operatorname{sur} G$ :

$$
d_{\mathbf{0}}^{\prime}(\gamma)=\left(\left(\begin{array}{cc}
0 & -\gamma^{*-1} \\
\gamma & 0
\end{array}\right),\left\langle u,-u^{*}\right\rangle\right)
$$

Soient encore $\alpha=1, \delta=1, \gamma=0 ; \sigma \sigma^{\mathrm{I}}=1$ se réduit alors à $\beta=\beta^{*}$, et les formules du $\mathbf{n}^{\circ} \mathbf{5}$ montrent que $f$ est de la forme $g(u) h\left(u^{*}\right)$, où $h$ est un caractère de $G^{*}$ et $g$ un caractère du second degré de $G$ associé à $\beta$. Cela conduit à poser, chaque fois que $f$ est un caractère du second degré de $G$, et que $\varrho$ est le morphisme symétrique de $G$ dans $G^{*}$ associé à $f$ :

$$
t_{0}(f)=\left(\left(\begin{array}{ll}
1 & \varrho \\
0 & 1
\end{array}\right), f\right)
$$

$f \rightarrow t_{0}(f)$ est alors un monomorphisme du groupe $X_{2}(G)$ des caractères du second degré de $G$ dans le groupe $B_{0}(G)$. De même, si $f^{\prime}$ est un caractère du second degré de $G^{*}$, associé au morphisme symétrique $\varrho^{\prime}$ de $G^{*}$ dans $G$, on écrira

$$
t_{0}^{\prime}\left(f^{\prime}\right)=\left(\left(\begin{array}{ll}
1 & 0 \\
\varrho & 1
\end{array}\right), f^{\prime}\right)
$$

ce qui définit un monomorphisme de $X_{2}\left(G^{*}\right)$ dans $B_{0}(G)$. 
Si $f$ est un caractère du second degré $\operatorname{de} G$, et $\alpha$ un automorphisme de $G$, on conviendra de poser

$$
f^{\alpha}(x)=f\left(x \alpha^{-1}\right)
$$

(cependant, comme cette notation prêterait à confusion pour $\alpha=-1$, on écrira $f-(x)=$ $f(-x))$; avec cette notation, on a

$$
d_{0}(\alpha)^{-1} t_{0}(f) d_{0}(\alpha)=t_{0}\left(f^{\alpha}\right), \quad d_{0}(\alpha) t_{0}^{\prime}\left(f^{\prime}\right) d_{0}(\alpha)^{-1}=t_{0}^{\prime}\left(f^{\prime \alpha^{*}}\right) .
$$

Si $\alpha$ est comme ci-dessus, et si $\gamma$ est un isomorphisme de $G^{*} \operatorname{sur} G$, on a

$$
d_{0}^{\prime}(\gamma \alpha)=d_{0}^{\prime}(\gamma) d_{0}(\alpha), \quad d_{0}^{\prime}\left(\alpha^{*-1} \gamma\right)=d_{0}(\alpha) d_{0}^{\prime}(\gamma)
$$

la première de ces relations montre en particulier que l'ensemble des éléments de $B_{0}(G)$ de la forme $d_{0}^{\prime}(\gamma)$, s'il n'est pas vide, est une classe à droite par rapport au sous-groupe de $B_{0}(G)$ formé par les éléments de la forme $d_{0}(\alpha)$. Plus généralement, on observera que, d'après (6), si un élément $s$ de $B_{0}(G)$ est de la forme $(\sigma, 1)$, le bicaractère $F$ doit être invariant par $\sigma$; comme $G^{*}$ est l'ensemble des $z_{1} \in G \times G^{*}$ tels que $F\left(z_{1}, z_{2}\right)=1$ quel que soit $z_{2}$, et que $G$ est l'ensemble des $z_{2} \in G \times G^{*}$ tels que $F\left(z_{1}, z_{2}\right)=1$ quel que soit $z_{1}$, il s'ensuit que $\sigma$ est alors de la forme $\left(\begin{array}{ll}\alpha & 0 \\ 0 & \delta\end{array}\right)$, et par suite, comme on l'a vu plus haut, qu'on a $s=d_{0}(\alpha)$; la formule (7) montre alors que, pour que deux éléments $s=(\sigma, f)$ et $s^{\prime \prime}=\left(\sigma^{\prime \prime}, f^{\prime \prime}\right)$ de $B_{0}(G)$ appartiennent à une même classe à droite suivant le sous-groupe des éléments de la forme $d_{0}(\alpha)$, il faut et il suffit que l'on ait $f=f^{\prime \prime}$.

7. Convenons désormais, pour $s=(\sigma, f)$ et $\sigma=\left(\begin{array}{ll}\alpha & \beta \\ \gamma & \delta\end{array}\right)$, de poser $\gamma=\gamma(s)$; et désignons par $\Omega_{0}(G)$ l'ensemble des $s \in B_{0}(G)$ tels que $\gamma(s)$ soit un isomorphisme de $G^{*}$ sur $G$ (cet ensemble pouvant être vide). On a alors le résultat suivant :

Proposition 1. L'ensemble $\Omega_{0}(G)$ des $s \in B_{0}(G)$ tels que $\gamma(s)$ soit un isomorphisme de $G^{*}$ sur $G$ est l'ensemble des éléments de $B_{0}(G)$ de la forme

$$
s=t_{0}\left(f_{1}\right) d_{0}^{\prime}(\gamma) t_{0}\left(f_{2}\right),
$$

où $\gamma$ est un isomorphisme de $G^{*}$ sur $G$ et où $f_{1}, f_{2}$ sont des caractères du second degré de $G$; et tout élément de $\Omega_{0}(G)$ se met sous cette forme d'une manière et d'une seule.

Si $s$ est donné par (8), on a $\gamma(s)=\gamma$, donc $s$ est dans $\Omega_{0}(G)$. Réciproquement, soit $s=(\sigma, f) \in \Omega_{0}(G)$; s'il est possible de satisfaire à $(8)$, on devra donc y prendre $\gamma=\gamma(s)$. Soit alors $\sigma=\left(\begin{array}{ll}\alpha & \beta \\ \gamma & \delta\end{array}\right)$; comme le montre un calcul facile, pour que (8) soit satisfait, il faut et il suffit que l'on ait 


$$
f_{1}(u)=f\left(u,-u \alpha \gamma^{-1}\right), \quad f_{2}(u)=f\left(0, u \gamma^{-1}\right)
$$

Cela démontre la proposition. On notera qu'en appliquant à (8) l'homomorphisme $s \rightarrow \sigma$, on obtient la relation

$$
\left(\begin{array}{ll}
\alpha & \beta \\
\gamma & \delta
\end{array}\right)=\left(\begin{array}{ll}
1 & \alpha \gamma^{-1} \\
0 & 1
\end{array}\right) \cdot\left(\begin{array}{cc}
0-\gamma^{*-1} \\
\gamma & 0
\end{array}\right) \cdot\left(\begin{array}{ll}
1 & \gamma^{-1} \delta \\
0 & 1
\end{array}\right)
$$

en raison de la symplecticité de $\sigma, \alpha \gamma^{-1}$ et $\gamma^{-1} \delta$ sont des morphismes symétriques de $G$ dans $G^{*}$; ils sont respectivement associés à $f_{1}$ et à $f_{2}$.

On obtient une relation importante en considérant un caractère du second degré $f$ non dégénéré de $G$, ce qui veut dire que le morphisme $\varrho$ associé à $f$ est un isomorphisme de $G$ sur $G^{*}$; alors la fonction $f^{\prime} \operatorname{sur} G^{*}$, définie par

$$
f^{\prime}\left(x^{*}\right)=f\left(-x^{*} \varrho^{-1}\right)
$$

est un caractère du second degré de $G^{*}$, associé au morphisme symétrique $\varrho^{-1}$ de $G^{*}$ sur $G$. La proposition 1 , appliquée à $t_{0}^{\prime}\left(f^{\prime}\right)$, donne

$$
t_{0}^{\prime}\left(f^{\prime}\right)=t_{0}(f) d_{0}^{\prime}\left(\varrho^{-1}\right) t_{0}\left(f^{-}\right)
$$

où $f^{-}$est défini par $f^{-}(x)=f(-x)$ comme on l'a dit plus haut. Un calcul facile donne d'autre part

$$
t_{0}^{\prime}\left(f^{\prime}\right)=d_{0}^{\prime}\left(\varrho^{-1}\right) t_{0}\left(f^{-1}\right) d_{0}^{\prime}\left(-\varrho^{-1}\right)
$$

Comme en même temps on a $d_{0}^{\prime}\left(\varrho^{-1}\right)^{2}=d_{0}(-1)$, on tire de là la relation que nous avions en vue :

$$
d_{0}^{\prime}\left(-\varrho^{-1}\right) t_{0}(f) d_{0}^{\prime}\left(\varrho^{-1}\right) t_{0}\left(f^{-}\right)=t_{0}\left(f^{-1}\right) d_{0}^{\prime}\left(-\varrho^{-1}\right)
$$

En tenant compte des relations obtenues au $n^{\circ} 6$, on aurait pu mettre (9) sous la forme plus simple

$$
\left(t_{0}(f) d_{0}^{\prime}\left(-\varrho^{-1}\right)\right)^{3}=e
$$

où $e$ est l'élément neutre de $B_{0}(G)$; sous cette forme, elle est bien connue dans la théorie classique du groupe modulaire. Mais c'est la relation (9), telle qu'elle se trouve écrite cidessus, que nous aurons à utiliser plus loin.

8. Les automorphismes du groupe $\mathbf{A}(G)$, isomorphe à $A(G)$, qui a été introduit au $\mathrm{n}^{\circ} 4$, sont bien entendu les mêmes que ceux de $A(G)$. Nous nous proposons maintenant de démontrer que tout automorphisme $s \in B_{0}(G)$ de $\mathbf{A}(G)$ est induit sur $\mathbf{A}(G)$ par un automorphisme intérieur du groupe de tous les opérateurs unitaires. Ce théorème est dû à $I$. Segal [6] dans le cas où $x \rightarrow 2 x$ est un automorphisme de $G$, et nous lui empruntons sa méthode 
de démonstration, qui consiste à introduire une algèbre d'opérateurs naturellement associée au groupe $\mathbf{A}(G)$. Pour cela, on posera, en un sens qui va être précisé dans un instant :

$$
U(\varphi)=\int U(w) \varphi(w) d w
$$

où $\varphi$ désigne une fonction sur $G \times G^{*}$, et où $w=\left(u, u^{*}\right.$ ) et $d w=d u \cdot d u^{*}$ (mesure qui ne dépend pas du choix de la mesure $d u$ sur $G$ ). Autrement dit, si $\Phi$ est une fonction sur $G, U(\varphi) \Phi$ est la fonction définie par

$$
U(\varphi) \Phi(x)=\int U(w) \Phi(x) \cdot \varphi(w) d w=\int \Phi(x+u) \cdot\left\langle x, u^{*}\right\rangle \cdot \varphi\left(u, u^{*}\right) d u d u^{*}
$$

où nous supposerons provisoirement, pour fixer les idées, que $\varphi$ et $\Phi$ sont toutes deux continues à support compact. Cela s'écrit aussi

$$
U(\varphi) \Phi(x)=\int K(x, y) \Phi(y) d y
$$

où $K$ est donné par

$$
K(x, y)=\int \varphi\left(y-x, u^{*}\right) \cdot\left\langle x, u^{*}\right\rangle \cdot d u^{*},
$$

ou, ce qui revient au même

$$
K(x, x+u)=\int \varphi\left(u, u^{*}\right) \cdot\left\langle x, u^{*}\right\rangle \cdot d u^{*}
$$

on obtient donc $K(-x,-x+u)$ à partir de $\varphi\left(u, u^{*}\right)$ en appliquant, pour chaque valeur de $u$, la transformation de Fourier à $\varphi\left(u, u^{*}\right)$ considérée comme fonction de $u^{*}$. Dans les conditions de validité de la formule d'inversion de la transformation de Fourier, on aura done:

$$
\varphi\left(u, u^{*}\right)=\int K(x, x+u) \cdot\left\langle x,-u^{*}\right\rangle \cdot d x
$$

de plus, en vertu du théorème de Plancherel, on a

$$
\int|K(x, y)|^{2} d x d y=\int\left|\varphi\left(u, u^{*}\right)\right|^{2} d u d u^{*}
$$

ce qui montre que la correspondance entre les fonctions $\varphi$ sur $G \times G^{*}$ et les fonctions $K$ sur $G \times G$, définie par les formules ci-dessus, se prolonge par continuité à un isomorphisme $W$ de $L^{2}\left(G \times G^{*}\right)$ sur $L^{2}(G \times G)$.

Lorsque $K$ est la fonction définie par $K(x, y)=P(x) Q(y)$, nous écrivons $K=P \otimes Q$; et, si 
$P$ et $Q$ sont dans $L^{2}(G)$, nous écrivons $(P, Q)=\int P(x) \overline{Q(x)} d x$; avec ces notations, les formules ci-dessus donnent en particulier

$$
W^{-1}(P \otimes \bar{Q})(w)=(P, U(w) Q) .
$$

9. Soient maintenant $\varphi_{1}, \varphi_{2}$ deux fonctions sur $G \times G^{*}$, que provisoirement nous supposerons continues à support compact; d'après (10), nous aurons

$$
U\left(\varphi_{1}\right) U\left(\varphi_{2}\right)=U\left(\varphi_{3}\right)
$$

où $\varphi_{3}$ est donné par la formule

$$
\varphi_{3}(w)=\int \varphi_{1}\left(w-w_{1}\right) \varphi_{2}\left(w_{1}\right) F\left(w-w_{1}, w_{1}\right) d w_{1}
$$

comme précédemment, $F$ désigne ici la fonction définie par (3) au $\mathrm{n}^{\circ} 3$. Si on pose $K_{i}=W\left(\varphi_{i}\right)$ pour $i=1,2,3,(11)$ montre que $K_{3}$ est donné par

$$
K_{3}(x, y)=\int K_{1}(x, z) K_{2}(z, y) d z
$$

ce que nous conviendrons d'écrire $K_{3}=K_{1} \times K_{2}$. De plus, les formules ci-dessus se prolongent par continuité aux espaces $L^{2}\left(G \times G^{*}\right), L^{2}(G \times G)$. Nous aurons besoin du lemme suivant :

LEM $\mathrm{E}$ 2. Soit $K \in L^{2}(G \times G)$; pour que $K$ soit de la forme $P \otimes Q$, avec $P$ et $Q$ dans $L^{2}(G)$, il faut et il suffit que, pour tout $K^{\prime} \in L^{2}(G \times G), K \times K^{\prime} \times K$ ne diffère de $K$ que par un facteur scalaire. Soient $K=P \otimes Q, K^{\prime}=P^{\prime} \otimes Q^{\prime}$, avec $P, Q, P^{\prime}, Q^{\prime}$ dans $L^{2}(G)$; pour que $P$ et $P^{\prime}$ (resp. $Q$ et $\left.Q^{\prime}\right)$ ne diffèrent l'un de l'autre que par un facteur scalaire, il faut et il suffit que, pour tout $K^{\prime \prime}=P^{\prime \prime} \otimes Q^{\prime \prime}$ avec $P^{\prime \prime}$ et $Q^{\prime \prime}$ dans $L^{2}(G), K \times K^{\prime \prime}$ et $K^{\prime} \times K^{\prime \prime}\left(\right.$ resp. $K^{\prime \prime} \times K$ et $\left.K^{\prime \prime} \times K^{\prime}\right)$ ne diffèrent l'un de l'autre que par un facteur scalaire.

La deuxième partie est évidente, et il est évident aussi que, dans la première partie, la condition énoncée est nécessaire; pour voir qu'elle est suffisante, il suffit de l'appliquer au cas où on a pris $K^{\prime}=P^{\prime} \otimes Q^{\prime}$

La seule conséquence de ce lemme dont nous ayons besoin est la suivante :

LEMME 3. Soit $K \rightarrow K^{s}$ un automorphisme de l'espace hilbertien $L^{2}(G \times G)$ muni de la loi de composition $\left(K_{1}, K_{2}\right) \rightarrow K_{1} \times K_{2}$ définie par (14). Alors il y a un automorphisme $t$ de $L^{2}(G)$ tel que l'on ait, quels que soient $P$ et $Q$ dans $L^{2}(G),(P \otimes Q)^{s}=P^{t} \otimes Q^{\bar{t}}$, où $\bar{t}$ est l" "imaginaire conjugué » de $t$, défini par $\bar{Q}^{\bar{t}}=\overline{\left(Q^{t}\right)}$. 
En effet, d'après le lemme 2, tout élément $(P \otimes Q)^{s}$ de $L^{2}(G \times G)$ est de la forme $P^{\prime} \otimes Q^{\prime}$. Choisissons $P_{0}$ tel que $\left\|P_{0}\right\|=1$; comme $s$ conserve la norme, on peut mettre $\left(P_{0} \otimes \bar{P}_{0}\right)^{s}$ sous la forme $P_{0}^{\prime} \otimes Q_{0}^{\prime}$ avec $\left\|P_{0}^{\prime}\right\|=\left\|Q_{0}^{\prime}\right\|=1$. La seconde partie du lemme 2 montre alors que, quels que soient $P, Q$ dans $L^{2}(G),\left(P \otimes \bar{P}_{0}\right)^{s}$ et $\left(P_{0} \otimes Q\right)^{s}$ se mettent respectivement, d'une manière et d'une seule, sous la forme $P^{\prime} \otimes Q_{0}^{\prime}$ et $P_{0}^{\prime} \otimes Q^{\prime}$. Si on écrit $P^{\prime}=P^{t}, Q^{\prime}=Q^{u}$, il est clair que $t$, $u$ sont des applications linéaires de $L^{2}(G) \operatorname{dans} L^{2}(G)$ et que $P_{0}^{t}=P_{0}^{\prime}, \bar{P}_{0}^{u}=Q_{0}^{\prime}$; comme $s$ conserve la norme dans $L^{2}(G \times G)$, il en est de même de $t$ et de $u$ dans $L^{2}(G)$. Comme on a $P \otimes Q=\left(P \otimes \bar{P}_{0}\right) \times\left(P_{0} \otimes Q\right)$, il s'ensuit que $(P \otimes Q)^{s}=c \cdot P^{t} \otimes Q^{u}$, avec $c=\left(P_{0}^{\prime}, \bar{Q}_{0}^{\prime}\right) ;$ pour $P=P_{0}$, $Q=Q_{0}$, cela donne $c=1$. Comme on a

$$
(P \otimes Q) \times(P \otimes Q)=(P, \bar{Q}) \cdot P \otimes Q,
$$

on voit que, pour $P^{\prime}=P^{t}, Q^{\prime}=Q^{u}$, on a $\left(P^{\prime}, \bar{Q}^{\prime}\right)=(P, \bar{Q})$; il s'ensuit que $u=\bar{t}$. Enfin, comme $s^{-1}$ a les mêmes propriétés que $s, t$ et $u$ sont inversibles; ce sont donc des automorphismes de $L^{2}(G)$.

10. Soit $s=(\sigma, f)$ un automorphisme de $A(G)$ appartenant à $B_{0}(G)$; nous le faisons opérer sur $\mathbf{A}(G)$ de la manière évidente, au moyen de l'isomorphisme entre $A(G)$ et $\mathbf{A}(G)$ qui nous a servi à définir ces groupes au $n^{\circ} 4$; en particulier, le transformé de $U(w)$ par $s$ sera donc $U(w)^{s}=f(w) \cdot U(w \sigma)$. On en déduit aussitôt un automorphisme de l'algèbre des opérateurs $U(\varphi)$ introduits au $\mathrm{n}^{\circ} 8$ :

$$
U(\varphi)^{s}=\int U(w \sigma) f(w) \varphi(w) d w
$$

ce qu'on peut écrire $U(\varphi)^{s}=U\left(\varphi^{s}\right)$, où $\varphi^{s}$ est donnée par

$$
\varphi^{s}(w)=f\left(w \sigma^{-1}\right) \varphi\left(w \sigma^{-1}\right)
$$

Il s'ensuit que $\varphi \rightarrow \varphi^{s}$, qui est évidemment un opérateur unitaire dans $L^{2}\left(G \times G^{*}\right)$, laisse invariante la loi de composition (13); c'est ce qu'il est facile aussi, bien entendu, de vérifier directement. Par suite, si on écrit, dans ces conditions, $K=W(\varphi)$ et $K^{s}=W\left(\varphi^{s}\right)$, ou en d'autres termes si on définit une application $K \rightarrow K^{s}$ de $L^{2}(G \times G)$ dans $L^{2}(G \times G)$ par la formule

$$
W^{-1}\left(K^{s}\right)=\left(W^{-1}(K)\right)^{s}
$$

cette application satisfera aux hypothèses du lemme 3 . D'après ce lemme, il y a donc un automorphisme $t$ de $L^{2}(G)$ tel que l'on ait, quels que soient $P, Q$ dans $L^{2}(G),(P \otimes Q)^{s}=$ $P^{t} \otimes Q^{\bar{t}}$. Changeant maintenant de notations, écrivons $P \rightarrow \mathrm{s}^{-1} P$ au lieu de $P \rightarrow P^{t}$; remplaçons $Q$ par $\bar{Q}$, et appliquons (12); cela donne: 


$$
(P, U(w) Q)^{s}=\left(\mathbf{s}^{-1} P, U(w) \mathbf{s}^{-1} Q\right)
$$

Par définition de $\varphi^{s}$, le premier membre a la valeur

$$
f\left(w \sigma^{-1}\right) \cdot\left(P, U\left(w \sigma^{-1}\right) Q\right)=\left(P, f\left(w \sigma^{-1}\right)^{-1} U\left(w \sigma^{-1}\right) Q\right)
$$

puisque $(P, Q)$ est antilinéaire en $Q$ et que $f$ prend ses valeurs dans $T$; et le second membre est égal à $\left(P, \mathrm{~s} U(w) \mathrm{s}^{-1} Q\right)$ puisque s est unitaire. Comme la relation obtenue est valable quels que soient $P, Q$, on a donc

$$
f\left(w \sigma^{-1}\right)^{-1} U\left(w \sigma^{-1}\right)=\mathrm{s} U(w) \mathrm{s}^{-1}
$$

d'où, en remplaçant $w$ par $w \sigma$ :

$$
\mathrm{s}^{-1} U(w) \mathrm{s}=f(w) \cdot U(w \sigma)=U(w)^{s} .
$$

Cela revient à dire que l'automorphisme intérieur déterminé par s dans le groupe unitaire induit sur $\mathbf{A}(G)$ l'automorphisme $s$. Réciproquement, $s$ étant donné, cette relation détermine s à un élément près du centralisateur de $\mathbf{A}(G)$. Mais, si un opérateur unitaire est permutable avec tous les $U(w)$, il l'est aussi avec tous les $U(\varphi)$, donc avec les opérateurs de la forme (11) quel que soit $K \in L^{2}(G \times G)$. Pour $K=P \otimes \bar{Q}$, (11) définit l'opérateur $\Phi \rightarrow(\Phi, Q) \cdot P$; si $\Phi \rightarrow \Phi^{t}$ est permutable avec celui-ci, on aura donc

$$
(\Phi, Q) \cdot P^{t}=\left(\Phi^{t}, Q\right) \cdot P
$$

quels que soient $P, Q, \Phi$ dans $L^{2}(G)$; donc $\Phi \rightarrow \Phi^{t}$ est de la forme $\Phi \rightarrow t \cdot \Phi$, où $t$ est un scalaire; si cet opérateur est unitaire, on a $t \in T$. On notera $\mathbf{T}$ le groupe formé par les opérateurs de cette forme; c'est le centre de $\mathbf{A}(G)$, et c'est aussi le centre du groupe de tous les automorphismes de $L^{2}(G)$. On a donc démontré le théorème suivant:

ThÉoRìme I. Le centralisateur de $\mathbf{A}(G)$ dans le groupe des automorphismes de $L^{2}(G)$ est le centre $\mathbf{T}$ de ces deux groupes; de plus, si $\mathbf{B}_{\mathbf{0}}(G)$ est le normalisateur de $\mathbf{A}(G)$ dans le même groupe, tout automorphisme de $\mathbf{A}(G)$ induisant l'identité sur $\mathbf{T}$ est induit sur $\mathbf{A}(G)$ par l'automorphisme intérieur déterminé par un élément de $\mathbf{B}_{0}(G)$; et $\mathbf{B}_{0}(G) / \mathbf{T}$ est isomorphe $\grave{a} B_{0}(G)$, c'est-à-dire au groupe des automorphismes de $A(G)$ induisant l'identité sur $T$.

11. On sait que la transformation de Fourier induit un automorphisme sur un certain espace de fonctions continues (dites, assez improprement, "indéfiniment différentiables à décroissance rapide "); cet espace $\mathfrak{S}(G)$ a même été introduit avant tout pour cette raison, par L. Schwartz ([4], Chap. VII) dans le cas de $\mathbf{R}^{n}$ et par F. Bruhat [1] dans le cas général. Nous allons voir que les opérateurs de $\mathbf{B}_{\mathbf{0}}(G)$ ont la même propriété. 
Rappelons la définition de $\boldsymbol{S}(G)$ pour un groupe abélien localement compact $G$. Considérons d'abord un groupe "élémentaire ", c'est-à-dire de la forme $G=\mathbf{R}^{n} \times \mathbf{Z}^{p} \times T^{\alpha} \times F$, où $F$ est un groupe fini. Une fonction polynome sur $G$ sera, par définition, une fonction qui peut s'écrire comme polynome par rapport aux coordonnées relatives aux facteurs $\mathbf{R}$ et $\mathbf{Z}$ dans le produit $G ; S(G)$ sera alors l'ensemble des fonctions $\Phi$, indéfiniment différentiables sur $G$, telles que $P \cdot D \Phi$ soit borné sur $G$ quels que soient l'opérateur différentiel invariant par translation $D$ et la fonction polynome $P$; la topologie de $S(G)$ est celle qui se déduit de l'ensemble des seminormes sup $|P \cdot D \Phi|$. Dans le cas général, on introduira tous les couples $\left(H, H^{\prime}\right)$ de sous-groupes de $G$ ayant les propriétés suivantes : (i) $H$ est engendré par un voisinage compact de 0 dans $G$ (il est donc ouvert et fermé dans $G$ ); (ii) $H^{\prime}$ est un sous-groupe compact de $H$, et $H / H^{\prime}$ est isomorphe à un groupe élémentaire. A un tel couple, on fait correspondre la famille $S\left(H, H^{\prime}\right)$ des fonctions continues sur $G$, à support contenu dans $H$, constantes sur les classes suivant $H^{\prime}$, et telles que la fonction sur $H / H^{\prime}$ qui s'en déduit par restriction à $H$ et passage au quotient appartienne à $S\left(H / H^{\prime}\right)$. Alors $S(G)$ est la réunion des $S\left(H, H^{\prime}\right)$; on lui donne la topologie "limite inductive " de celles des $S\left(H / H^{\prime}\right)$, c'est-à-dire qu'un ensemble convexe $X$ est un voisinage de 0 dans $S(G)$ si, quel que soit le couple $\left(H, H^{\prime}\right)$, l'image de $X \cap S\left(H, H^{\prime}\right)$ dans $S\left(H / H^{\prime}\right)$ est un voisinage de 0 dans $S\left(H / H^{\prime}\right)$.

Nous nous proposons de faire voir que tout $\mathrm{s} \in \mathbf{B}_{\mathbf{0}}(G)$ induit sur $S(G)$ un automorphisme de $S(G)$; il suffit pour cela de montrer que $s$ induit sur $S(G)$ une application continue de $S(G)$ dans lui-même; c'est ce qu'on fera en suivant pas à pas la démonstration du théorème 1. Nous écrirons de nouveau $t$ au lieu de $\mathrm{s}^{-1}, P^{t}$ au lieu de $\mathrm{s}^{-1} P$ pour $P \in L^{2}(G)$, et nous ferons la démonstration pour l'opérateur $P \rightarrow P^{t}$. D'après ce qui précède, si on se donne $Q \neq 0$ dans $S(G)$, l'application $P \rightarrow P^{t}$ est la composée des suivantes :

$$
\begin{gathered}
\text { (a) } P \rightarrow K=P \otimes Q ; \quad(b) K \rightarrow \varphi=W^{-1}(K) ; \quad(c) \varphi \rightarrow \varphi^{s} ; \\
\text { (d) } \varphi^{s} \rightarrow K^{s}=W\left(\varphi^{s}\right) ; \quad \text { (e) } K^{s}=P^{t} \otimes Q^{\bar{t}} \rightarrow P^{t} ;
\end{gathered}
$$

il suffit donc de montrer que celles-ci font passer de $S(G)$ à $S(G \times G)$, puis à $S\left(G \times G^{*}\right)$, puis à $S\left(G \times G^{*}\right)$, puis à $S(G \times G)$, puis à $S(G)$, chaque fois de façon continue. Pour (a), c'est immédiat; c'est immédiat aussi pour (e), à condition de remarquer que, si une fonction $K \in S(G \times G)$ est de la forme $P \otimes Q$ au sens de $L^{2}(G \times G)$, elle est de la forme $P \otimes Q$ avec $P$ et $Q$ dans $S(G)$, et que, pour $Q \neq 0$ dans $S(G)$, l'application $P \rightarrow P \otimes Q$ est un isomorphisme de $S(G)$ sur un sous-espace fermé de $S(G \times G)$. Pour $(b)$ et $(d)$, il s'agit de montrer que $W$ détermine un isomorphisme de $\mathcal{S}\left(G \times G^{*}\right)$ sur $\mathcal{S}(G \times G)$; or $W$ est composé de l'opérateur $F(x, y) \rightarrow F(y-x,-x)$, qui détermine évidemment un automorphisme de $S(G \times G)$, et de la transformation de Fourier partielle relative au second facteur du produit $G \times G^{*}$. On 
est donc ramené à vérifier que, si $A$ et $B$ sont des groupes abéliens localement compacts, et si $B^{*}$ est le dual de $B$, la transformation de Fourier partielle

$$
f(a, b) \rightarrow f^{\prime}\left(a, b^{*}\right)=\int f(a, b) \cdot\left\langle b, b^{*}\right\rangle d b
$$

détermine un isomorphisme de $\mathfrak{S}(A \times B)$ sur $\mathfrak{S}\left(A \times B^{*}\right)$. C'est là une généralisation facile du théorème analogue sur la transformation de Fourier ordinaire.

12. Il nous reste à considérer $(c)$. Comme un automorphisme $\sigma$ de $G \times G^{*}$ détermine évidemment un automorphisme de $S\left(G \times G^{*}\right)$, nous sommes donc (après avoir substitué $G$ à $\left.G \times G^{*}\right)$ ramenés à démontrer ce qui suit :

Proposition 2. Soit $f$ un caractère du second degré de $G$. Alors $\Phi \rightarrow \Phi f$ est un automorphisme de $S(G)$.

Soit d'abord $G=\mathbf{R}^{n} \times \mathbf{Z}^{p} \times T^{q} \times F$, avec $F$ fini; comme on le voit facilement, tout revient à montrer que, pour tout opérateur différentiel $D$ invariant par translation sur $G$, il y a une fonction polynome $P$ sur $G$ telle que $|D f| \leqslant|P|$. C'est ce qu'on vérifie sans difficulté en exprimant $f$ sur les classes suivant $\mathbf{R}^{n} \times T^{q}$ dans $G$ au moyen de la formule (1) du $\mathbf{n}^{\circ} 1$, et en remarquant que, sur $\mathbf{R}^{n} \times T^{a}$, $f$ est nécessairement de la forme $e^{i F(x)} \chi(x, y)$, où $x \in \mathbf{R}^{n}, y \in T^{q}, F$ étant une forme quadratique sur $\mathbf{R}^{n}$ et $\chi$ étant un caractère de $\mathbf{R}^{n} \times T^{q}$. Passons au cas général; soit $\varrho$ le morphisme symétrique de $G$ dans $G^{*}$ associé à $f$, et donnons-nous un sous-groupe $H$ de $G$ engendré par un voisinage compact de 0. Pour qu'un sous-groupe $H^{\prime}$ de $H$ satisfasse à la condition (ii) de la définition de $S\left(H, H^{\prime}\right)$, il faut et il suffit, comme on sait (cf. [1], $n^{\circ} 9$, p. 60), que le groupe $H_{*}^{\prime}$ qui lui est associé par dualité dans $G^{*}$ (l' " orthogonal " de $H^{\prime}$ ) soit engendré par un voisinage compact de 0; alors le groupe $H_{*}^{\prime}+H \varrho$ aura la même propriété, ce qui montre qu'en remplaçant $H_{*}^{\prime}$ par celui-ci, done $H^{\prime}$ par un groupe plus petit qui satisfait aussi à (ii), on peut faire en sorte qu'on ait $H \varrho \subset H_{*}^{\prime}$. D'après (1) du $\mathrm{n}^{\circ} 1$, cela donne $f\left(h+h^{\prime}\right)=f(h) f\left(h^{\prime}\right)$ chaque fois que $h \in H, h^{\prime} \in H^{\prime}$. En particulier, $f$ induit alors sur $H^{\prime}$ un caractère de $H^{\prime}$, qu'on peut, en le prolongeant à un caractère de $G$, écrire sous la forme $\left\langle h^{\prime}, a^{*}\right\rangle$ avec $a^{*} \in G^{*}$. En remplaçant $H_{*}^{\prime}$ par le groupe engendré par $H_{*}^{\prime}$ et $a^{*}$, on peut alors faire en sorte que $a^{*}$ soit dans $H_{*}^{\prime}$; cela fait, $f$ induit la constante 1 sur $H^{\prime}$ et est constante dans $H$ sur toute classe suivant $H^{\prime}$. D'après ce qu'on a démontré dans le cas où $G$ est un groupe élémentaire, il s'ensuit, par passage au quotient, que $\Phi \rightarrow \Phi f$ détermine un automorphisme de $\mathfrak{S}\left(H, H^{\prime}\right)$. Comme il en est ainsi, pour tout $H$ satisfaisant à (i), pourvu que $H^{\prime}$ ait été pris assez petit et satisfasse à (ii), cela achève la démonstration (compte tenu de la définition de la topologie dans $\boldsymbol{S}(G)$ comme limite inductive).

11-642946 Acta mathematica. 111. Imprimé le 3 juin 1964. 
13. L'homomorphisme $\mathbf{s} \rightarrow s=(\sigma, f)$ de $\mathbf{B}_{0}(G)$ sur $B_{0}(G)$ déterminé par (15) sera noté $\pi_{0}$ et sera appelé la projection canonique du premier groupe sur le second. En général (comme le montrera plus loin l'exemple des groupes de type local), il n'existe pas de section de $\mathbf{B}_{0}(G)$ au-dessus de $B_{0}(G)$ qui soit en même temps un sous-groupe de $\mathbf{B}_{0}(G)$. Mais il est très utile de savoir qu'on peut du moins définir des sections au-dessus des sous-groupes et sous-ensembles de $B_{0}(G)$ qui ont été introduits aux $n^{\text {os }} 6$ et 7 .

Soit $\Phi \in L^{2}(G)$. Pour tout automorphisme $\alpha$ de $G$, on posera

$$
\mathbf{d}_{0}(\alpha) \Phi(x)=|\alpha|^{\frac{1}{2}} \Phi(x \alpha)
$$

Pour tout caractère du second degré $f$ de $G$, on posera

$$
\mathbf{t}_{0}(f) \Phi(x)=\Phi(x) f(x)
$$

Pour tout isomorphisme $\gamma$ de $G^{*}$ sur $G$, on posera

$$
\mathrm{d}_{0}^{\prime}(\gamma) \Phi(x)=|\gamma|^{-\frac{1}{2}} \Phi^{*}\left(-x \gamma^{*-1}\right),
$$

où, comme précédemment, $\Phi^{*}$ désigne la transformée de Fourier de $\Phi$. On vérifie sans difficulté que $\mathbf{d}_{0}, \mathbf{t}_{\mathbf{0}}, \mathbf{d}_{0}^{\prime}$ sont des « relèvements " à $\mathbf{B}_{0}(G)$ des applications $d_{0}, t_{0}, d_{0}^{\prime}$ définies au $n^{\circ} 6$, c'est-à-dire qu'on a $d_{0}=\pi_{0} \circ d_{0}, t_{0}=\pi_{0} \circ t_{0}, d_{0}^{\prime}=\pi_{0} \circ d_{0}^{\prime}$. De plus, $d_{0}$ et $t_{0}$ sont des monomorphismes, dans $\mathbf{B}_{0}(G)$, du groupe des automorphismes de $G$, et du groupe $X_{2}(G)$, respectivement; et, quand $\alpha, f, \gamma$ sont comme ci-dessus, on a :

$$
\mathbf{d}_{0}(\alpha)^{-1} \mathbf{t}_{0}(f) \mathbf{d}_{\mathbf{0}}(\alpha)=\mathbf{t}_{\mathbf{0}}\left(f^{\alpha}\right), \quad \mathbf{d}_{0}^{\prime}(\gamma \alpha)=\mathbf{d}_{0}^{\prime}(\gamma) \mathbf{d}_{\mathbf{0}}(\alpha), \quad \mathbf{d}_{0}^{\prime}\left(\alpha^{*-1} \gamma\right)=\mathbf{d}_{0}(\alpha) \mathbf{d}_{0}^{\prime}(\gamma) .
$$

La proposition $1 \mathrm{du} \mathrm{n}^{\circ} 7$ permet alors de « relever " à $\mathbf{B}_{0}(G)$ tout élément de l'ensemble $\Omega_{0}(G)$ défini dans cette proposition. En effet, d'après celle-ci, tout $s \in \Omega_{0}(G)$ se met d'une manière et d'une seule sous la forme (8); $s$ étant donné par (8), nous poserons

$$
\mathbf{r}_{\mathbf{0}}(s)=\mathbf{t}_{\mathbf{0}}\left(f_{1}\right) \mathbf{d}_{0}^{\prime}(\gamma) \mathbf{t}_{\mathbf{0}}\left(f_{2}\right) \text {. }
$$

Un calcul facile permet d'expliciter cette formule; en écrivant, comme d'habitude, $s=(\sigma, f), \sigma=\left(\begin{array}{ll}\alpha & \beta \\ \gamma & \delta\end{array}\right)$, on obtient

$$
\mathbf{r}_{0}(s) \Phi(x)=|\gamma|^{\frac{1}{2}} \int \Phi\left(x \alpha+x^{*} \gamma\right) f\left(x, x^{*}\right) d x^{*}
$$

Les conditions de validité de cette formule sont évidemment les mêmes que celles de la formule de "définition " de la transformation de Fourier, qui a servi à expliciter $\mathbf{d}_{\mathbf{0}}$; par exemple, elle est valable presque partout si $\Phi \in L^{2}(G) \cap L^{1}(G)$; elle est valable pour tout $x$ si $\Phi \in \boldsymbol{S}(G)$, les deux membres définissant alors une même fonction de $\boldsymbol{S}(G)$. 
14. On obtient des résultats importants en "relevant " à $\mathbf{B}_{\mathbf{0}}(G)$, au moyen des applications $d u n^{\circ} 13$, des relations entre éléments de $B_{0}(G)$; c'est ce que nous allons faire d'abord pour la relation (9) du $n^{\circ} 7$. Comme au $n^{\circ} 7$, nous considérons done un caractère du second degré $f$ non dégénéré de $G$, associé à l'isomorphisme symétrique $\varrho$ de $G$ sur $G^{*}$. Désignons pour un instant par $s$ et $s^{\prime}$ les opérateurs qui se déduisent respectivement du premier et du second membre de (9) lorsqu'on y substitue $d_{0}^{\prime}, \mathbf{t}_{0}$ à $d_{0}^{\prime}$ et $t_{0}$. D'autre part, $\Phi$ étant provisoirement supposée continue à support compact, posons $\Phi_{1}=\Phi * f$, cette notation désignant naturellement le produit de composition usuel

$$
\Phi_{1}(x)=\int \Phi(u) f(x-u) d u
$$

alors un calcul facile montre que $\mathbf{s} \Phi, \mathrm{s}^{\prime} \Phi$ sont données par

$$
\mathbf{s} \Phi(x)=|\varrho| \Phi_{1}^{*}(x \varrho), \quad \mathbf{s}^{\prime} \Phi(x)=|\varrho|^{\frac{1}{2}} \Phi *(x \varrho) \cdot f(x)^{-1} .
$$

Les opérateurs introduits ici étant tous unitaires, il s'ensuit que l'opérateur $\Phi \rightarrow \Phi * f$ est continu au sens de $L^{2}(G)$. De plus, la relation (9), qui s'écrit maintenant $\pi_{0}(s)=\pi_{0}\left(\mathbf{s}^{\prime}\right)$, implique que $\mathbf{s}$ et $\mathbf{s}^{\prime}$ ne peuvent différer que par un facteur scalaire de valeur absolue 1 . Nous écrirons $\mathrm{s}=\gamma(f) \mathrm{s}^{\prime}$, ce qui donne, en substituant $x^{*} \varrho^{-1}$ à $x$ :

$$
\mathcal{T}(\Phi * f)=\gamma(f)|\varrho|^{-\frac{1}{2}} \mathcal{T}(\Phi) \cdot g
$$

où $g$ est le caractère du second degré de $G^{*}$, associé à $-\varrho^{-1}$, qui est défini par

$$
g\left(x^{*}\right)=f\left(x^{*} \underline{Q}^{-1}\right)^{-1} \text {. }
$$

Conformément aux conventions usuelles dans la théorie de la transformation de Fourier, on exprime (17) en disant que $\gamma(f)|\varrho|^{-\frac{1}{2}} g$ est la transformée de Fourier de $f$. Nous avons ainsi démontré ce qui suit :

THÉ OR ìme 2. Soit $f$ un caractère du second degré non dégénéré de $G$, associé à l'isomorphisme symétrique $\varrho$ de $G$ sur $G^{*}$. Alors $f$ possède une transformée de Fourier $\mathfrak{J}(f)$, donnée par la formule

$$
\mathcal{T}(f)\left(x^{*}\right)=\gamma(f)|\varrho|^{-\frac{1}{2}} f\left(x^{*} \varrho^{-1}\right)^{-1},
$$

où $\gamma(f)$ est un facteur scalaire de valeur absolue 1.

Répétons que cette assertion doit être entendue dans le sens suivant : l'application $\Phi \rightarrow \Phi * f$ se prolonge par continuité à $L^{2}(G)$, et, pour tout $\Phi \in L^{2}(G)$, on a $\mathfrak{T}(\Phi * f)=\Phi^{*} \cdot \mathcal{J}(f)$. Par transport de structure au moyen de l'isomorphisme $\varrho$ de $G$ sur $G^{*}$, on en conclut qu'alors on a aussi $\mathcal{J}(\Phi f)=\Phi^{*} * \mathcal{J}(f)$. Au moyen de la proposition $2 \mathrm{du}^{\circ} 12$, on voit de plus que, 
A. WEIL

pour $\Phi \in S(G)$, les deux membres de cette dernière relation sont des fonctions continues, done que l'égalité a lieu, non seulement au sens de $L^{2}\left(G^{*}\right)$, mais en tout point, puis, par transport de structure, qu'il en est de même pour la relation précédente. Nous expliciterons ce résultat sous forme de corollaire :

CoRollair E 1. Les hypothèses et notations étant celles du théorème 2, soit de plus $\Phi \in S(G)$, et soit $\Phi^{*}=\mathcal{J}(\Phi)$. Alors on a, pour tout $x^{*} \in G^{*}$ :

$$
\int(\Phi * f)(x) \cdot\left\langle x, x^{*}\right\rangle \cdot d x=\gamma(f)|\varrho|^{-\frac{1}{2}} \Phi^{*}\left(x^{*}\right) f\left(x^{*} \varrho^{-1}\right)^{-1}
$$

En particulier, pour $x^{*}=0$, on obtient une formule qui, réciproquement, implique celle du corollaire 1 :

COROLLAIRE 2. Les hypothèses et notations étant celles du théorème 2, on a, pour toute fonction $\Phi \in \boldsymbol{S}(G)$ :

$$
\int\left(\int \Phi(x-y) f(y) d y\right) d x=\gamma(f)|\varrho|^{-\frac{1}{2}} \int \Phi(x) d x
$$

Les nombres $\gamma(f)$, attachés aux caractères du second degré d'un groupe $G$ en vertu du théorème 2, ont une grande importance en théorie des nombres. Dans le cas d'un groupe $G$ de type local, ce sont, comme on le verra plus loin, des racines huitièmes de l'unité, apparentées aux sommes de Gauss.

15. Le " relèvement » $r_{0}$ de $\Omega_{0}(G)$ dans $B_{0}(G)$, défini au $n^{\circ} 13$, amène à introduire comme

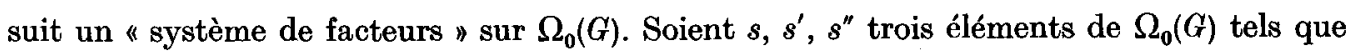
l'on ait $s^{\prime \prime}=s s^{\prime}$. Alors $\mathbf{r}_{0}(s) \mathbf{r}_{0}\left(s^{\prime}\right)$ a même image que $\mathbf{r}_{0}\left(s^{\prime \prime}\right)$ dans $B_{0}(G)$ et n'en diffère donc que par un facteur scalaire $\lambda\left(s, s^{\prime}\right) \in T$; c'est celui-ci qu'il s'agit maintenant de déterminer. Or, avec des notations évidentes, ce facteur est défini par

$$
\mathbf{t}_{\mathbf{0}}\left(f_{1}\right) \mathbf{d}_{0}^{\prime}(\gamma) \mathbf{t}_{0}\left(f_{2}\right) \cdot \mathbf{t}_{\mathbf{0}}\left(f_{1}^{\prime}\right) \mathbf{d}_{0}^{\prime}\left(\gamma^{\prime}\right) \mathbf{t}_{0}\left(f_{2}^{\prime}\right)=\lambda\left(s, s^{\prime}\right) \mathbf{t}_{\mathbf{0}}\left(f_{1}^{\prime \prime}\right) \mathbf{d}_{0}^{\prime}\left(\gamma^{\prime \prime}\right) \mathbf{t}_{\mathbf{0}}\left(f_{2}^{\prime \prime}\right)
$$

Posons $f_{0}=f_{2} f_{1}^{\prime}, f_{3}=f_{1}^{-1} f_{1}^{\prime \prime}, f_{4}=f_{2}^{\prime \prime} f_{2}^{\prime-1}$. On aura donc:

$$
\mathbf{d}_{0}^{\prime}(\gamma) \mathbf{t}_{0}\left(f_{0}\right) \mathbf{d}_{0}^{\prime}\left(\gamma^{\prime}\right)=\lambda\left(s, s^{\prime}\right) \mathbf{t}_{0}\left(f_{3}\right) \mathbf{d}_{0}^{\prime}\left(\gamma^{\prime \prime}\right) \mathbf{t}_{0}\left(f_{4}\right)
$$

L'opérateur du premier membre de (18), appliqué à une fonction $\Phi$, donne

à condition de poser

$$
|\gamma|^{-\frac{1}{2}}\left|\gamma^{\prime}\right|^{-\frac{1}{2}} \Psi_{i}^{*}\left(-x \gamma^{*-1}\right)
$$

$$
\Psi(x)=\Phi^{*}\left(-x \gamma^{\prime *-1}\right), \Psi_{1}(x)=\Psi(x) f_{0}(x) \text {. }
$$

Mais $f_{0}$ est un caractère du second degré de $G$, associé au morphisme symétrique 


$$
\varrho=\varrho_{2}+\varrho_{1}^{\prime}=\gamma^{-1} \delta+\alpha^{\prime} \gamma^{\prime-1}=\gamma^{-1} \gamma^{\prime \prime} \gamma^{\prime-1},
$$

comme il résulte des formules du $n^{\circ} 7$; donc $f_{0}$ est non dégénéré, et sa transformée de Fourier est donnée par le théorème 2. La transformée de Fourier $\Psi_{1}^{*}$ est alors $\Psi^{*} * \mathcal{T}\left(f_{0}\right)$. Comme $\Psi^{*}$ est donnée par $\Psi^{*}\left(u^{*}\right)=\left|\gamma^{\prime}\right| \cdot \Phi\left(u^{*} \gamma^{\prime}\right)$, l'image de $\Phi$ par l'opérateur du premier membre de (18) est donc (compte tenu de ce que $\varrho=\varrho^{*}$ ) :

$$
\gamma\left(f_{0}\right) \cdot\left|\gamma^{\prime \prime}\right|^{-\frac{1}{2}} \int \Phi\left(u^{*} \gamma^{\prime}\right) f_{0}\left(-x \gamma^{*-1} \gamma^{*}-u^{*} \gamma^{\prime} \gamma^{\prime \prime-1} \gamma\right)^{-1} d\left(u^{*} \gamma^{\prime}\right)
$$

D'autre part, l'image de $\Phi$ par l'opérateur du second membre de (18) est

$$
\lambda\left(s, s^{\prime}\right) f_{3}(x) \cdot\left|\gamma^{\prime \prime}\right|^{-\frac{1}{2}} \int \Phi(u) f_{4}(u) \cdot\left\langle u,-x \gamma^{\prime \prime *-1}\right\rangle \cdot d u
$$

On peut vérifier, bien qu'assez laborieusement, que ces deux images ne diffèrent que par un facteur constant; mais cela résulte déjà des calculs ci-dessus, et c'est inutile pour notre objet, qui est seulement de déterminer $\lambda\left(s, s^{\prime}\right)$. Pour cela, il suffit d'observer qu'après avoir fait dans la première intégrale le changement de variable $u=u^{*} \gamma^{\prime}$, les deux intégrales apparaissent respectivement sous la forme $c_{1} \int \Phi(u) g_{1}(x, u) d u, c_{2} \int \Phi(u) g_{2}(x, u) d u$, où $c_{1}, c_{2}$ sont des constantes et $g_{1}, g_{2}$ sont des caractères du second degré de $G \times G$. Pour qu'elles aient même valeur quel que soit $\Phi \in L^{2}(G)$, il faut évidemment que $c_{1}=c_{2}$ et $g_{1}=g_{2}$; or la première de ces égalités donne $\lambda\left(s, s^{\prime}\right)=\gamma\left(f_{0}\right)$. C'est là le résultat que nous avions en vue. En l'explicitant au moyen des formules du $n^{\circ} 7$ et du $n^{\circ} 13$, on peut l'énoncer comme suit :

ThÉoRème 3. Soient $s=(\sigma, f), s^{\prime}=\left(\sigma^{\prime}, f^{\prime}\right), s^{\prime \prime}=\left(\sigma^{\prime \prime}, f^{\prime \prime}\right)$ trois éléments de $B_{0}(G)$ tels que $s^{\prime \prime}=s s^{\prime}$; supposons que, lorsqu'on écrit $\sigma, \sigma^{\prime}, \sigma^{\prime \prime}$ sous la forme

$$
\sigma=\left(\begin{array}{ll}
\alpha & \beta \\
\gamma & \delta
\end{array}\right), \quad \sigma^{\prime}=\left(\begin{array}{ll}
\alpha^{\prime} & \beta^{\prime} \\
\gamma^{\prime} & \delta^{\prime}
\end{array}\right), \quad \sigma^{\prime \prime}=\left(\begin{array}{ll}
\alpha^{\prime \prime} & \beta^{\prime \prime} \\
\gamma^{\prime \prime} & \delta^{\prime \prime}
\end{array}\right),
$$

$\gamma, \gamma^{\prime}, \gamma^{\prime \prime}$ soient des isomorphismes de $G^{*}$ sur $G$. Alors la formule

$$
f_{0}(u)=f\left(0, u \gamma^{-1}\right) f^{\prime}\left(u,-u \alpha^{\prime} \gamma^{\prime-1}\right)
$$

définit un caractère du second degré non dégénéré de $G$, associé à l'isomorphisme symétrique $\gamma^{-1} \gamma^{\prime \prime} \gamma^{\prime-1}$ de $G$ sur $G^{*}$; et les opérateurs $\mathbf{r}_{0}(s), \mathbf{r}_{0}\left(s^{\prime}\right), \mathbf{r}_{0}\left(s^{\prime \prime}\right)$, respectivement associés à $s, s^{\prime}, s^{\prime \prime}$ au moyen de la formule (16), sont liés par la relation

$$
\mathbf{r}_{0}(s) \mathbf{r}_{0}\left(s^{\prime}\right)=\gamma\left(f_{0}\right) \mathbf{r}_{0}\left(s^{\prime \prime}\right),
$$

où $\gamma\left(f_{0}\right)$ est défini par le théorème 2. 
16. Soit maintenant $\Gamma$ un sous-groupe fermé de $G$, et soit $\Gamma_{*}$ le sous-groupe fermé de $G^{*}$ qui lui est associé par la théorie de la dualité, c'est-à-dire l'ensemble des $x^{*} \in G^{*}$ tels que $\left\langle\xi, x^{*}\right\rangle=1$ quel que soit $\xi \in \Gamma$; on peut alors, comme il est bien connu, identifier $\Gamma_{*}$ avec le dual de $G / \Gamma$, et $G^{*} / \Gamma_{*}$ avec celui de $\Gamma$. Signalons dès maintenant les cas qui intéressent la théorie des nombres; ce sont les suivants : $(a) G$ est de type local, c'est-à-dire est un espace vectoriel $X$ de dimension finie, sur un corps $k$ localement compact à valuation discrète, et $\Gamma$, pour un choix convenable d'une base de $X$, est le groupe des points dont les coordonnées sont des entiers de $k$; $(b) G$ est de type local sur $\mathbf{R}$ ou sur $\mathbf{C}$, et est donc un espace vectoriel de dimension finie sur $\mathbf{R}$, et $\Gamma$ est le sous-groupe engendré par une base de $G$ sur $\mathbf{R} ;(c) G$ est de type adélique, donc de la forme $X_{A}=X_{k} \otimes A_{k}$ (cf. $\mathrm{n}^{\circ}$ 1), et $\Gamma=X_{k}$. On notera que, dans ces trois cas, il existe des isomorphismes de $G$ sur $G^{*}$ qui transforment $\Gamma$ en $\Gamma_{*}$; dans le cas $(a), \Gamma$ et $\Gamma_{*}$ sont compacts à quotient discret, et dans les cas $(b)$ et $(c)$ ils sont discrets à quotient compact.

On notera $\dot{x}$ l'image dans $G / \Gamma$ d'un élément $x$ de $G$, et $\dot{x}^{*}$ l'image dans $G^{*} / \Gamma_{*}$ d'un élément $x^{*}$ de $G^{*}$. On choisira des mesures de Haar $d \xi$ dans $\Gamma$ et $d \dot{x}$ dans $G / \Gamma$ de manière à avoir l'égalité symbolique $d x=d \xi d \dot{x}$, ce qui signifie, comme on sait, qu'on a, pour toute fonction $\Phi \in L^{1}(G)$ :

$$
\int_{G} \Phi(x) d x=\int_{G / \Gamma}\left(\int_{\Gamma} \Phi(x+\xi) d \xi\right) d \dot{x}
$$

ici, comme dans tout ce qui suivra, la notation se justifie du fait que la fonction à intégrer sur $G / \Gamma$ au second membre, bien qu'écrite comme fonction de $x \in G$, est invariante par $x \rightarrow x+\xi$ pour tout $\xi \in \Gamma$ et peut donc être considérée, en un sens évident, comme fonction de $\dot{x} \operatorname{sur} G / \Gamma$. Pour toute fonction $\Phi$ sur $G$, et tout $x \in G$, on conviendra dans ce qui suit de noter $\Phi_{x}$ la fonction sur $\Gamma$ définie par $\Phi_{x}(\xi)=\Phi(x+\xi)$, de sorte que l'égalité ci-dessus s'écrit, en abrégé, $\int \Phi d x=\int\left(\int \Phi_{x} d \xi\right) d \dot{x}$. En y remplaçant $\Phi$ par $|\Phi|^{2}$, on obtient en particulier la relation

$$
\|\Phi\|_{G}^{2}=\int_{G / \Gamma}\left\|\Phi_{x}\right\|_{\Gamma}^{2} d \dot{x}
$$

où $\|\Phi\|_{G},\left\|\Phi_{x}\right\|_{\Gamma}$ dénotent les normes dans $L^{2}(G)$ et dans $L^{2}(\Gamma)$, respectivement. D'autre part, en procédant de même pour l'intégrale qui définit la transformée de Fourier d'une fonction $\Phi \in L^{1}(G)$, on est conduit à introduire la fonction $\Theta \operatorname{sur} G \times G^{*}$, définie par la formule

$$
\Theta\left(x, x^{*}\right)=\int_{\Gamma} \Phi(x+\xi) \cdot\left\langle\xi, x^{*}\right\rangle \cdot d \xi
$$

la transformée de Fourier $\Phi^{*}$ de $\Phi$ s'écrivant alors

$$
\Phi^{*}\left(x^{*}\right)=\int_{G / \mathrm{V}} \Theta\left(x, x^{*}\right) \cdot\left\langle x, x^{*}\right\rangle \cdot d \dot{x} .
$$


On notera que, si $\Gamma$ est discret, l'intégrale qui définit $\Theta$ se réduit à une somme. Dans les cas (b) et (c) mentionnés plus haut, les fonctions de cette forme ont une grande importance; en particulier, pour des choix convenables de $\Phi$, elles se réduisent à des séries thêta au sens classique.

17. Supposons provisoirement, pour fixer les idées, que $\Phi$ soit continue à support compact; alors $\Theta$ est continue. Quels que soient $\xi \in \Gamma, \xi^{*} \in \Gamma_{*}, \Theta$ satisfait à la relation

$$
\Theta\left(x+\xi, x^{*}+\xi^{*}\right)=\Theta\left(x, x^{*}\right) \cdot\left\langle\xi,-x^{*}\right\rangle,
$$

ce qui, en posant $z=\left(x, x^{*}\right), \zeta=\left(\xi, \xi^{*}\right)$, s'écrit aussi, au moyen de la fonction $F$ définie par (3) au $n^{\circ} 3$ :

$$
\Theta(z+\zeta)=\Theta(z) F(\zeta, z)^{-1} \quad\left(z \in G \times G^{*}, \zeta \in \Gamma \times \Gamma_{*}\right)
$$

En particulier, $\Theta$ est invariante par $x^{*} \rightarrow x^{*}+\xi^{*}$ quel que soit $\xi^{*} \in \Gamma_{*}$. Pour toute solution de (22), nous conviendrons de désigner par $\Theta_{x}$, pour $x \in G$, la fonction définie sur $G^{*} / \Gamma_{*}$ par $\Theta_{x}\left(\dot{x}^{*}\right)=\Theta\left(x, x^{*}\right)$. On peut alors exprimer (20) en disant que, pour tout $x$, $\Theta_{x}$ est la transformée de Fourier de $\Phi_{x}$.

Identifiant $G^{*} / \Gamma_{*}$ avec le dual de $\Gamma$, et $\Gamma_{*}$ avec celui de $G / \Gamma$, nous munirons ces groupes des mesures de Haar $d \dot{x}^{*}, d \xi^{*}$, respectivement duales de $d \xi$, $d \dot{x}$; pour $z=\left(x, x^{*}\right)$, nous désignerons par $\dot{z}$ l'image $\left(\dot{x}, \dot{x}^{*}\right)$ de $z$ dans le groupe

$$
Q=\left(G \times G^{*}\right) /\left(\Gamma \times \Gamma_{*}\right)=(G / \Gamma) \times\left(G^{*} / \Gamma_{*}\right),
$$

et nous poserons $d \dot{z}=d \dot{x} d \dot{x}^{*}$. D'après $(22),|\Theta|$ est invariant par $z \rightarrow z+\zeta$ quel que soit $\zeta \in \Gamma \times \Gamma_{*}$, et peut donc être considéré comme fonction sur $Q$. Le théorème de Plancherel, appliqué à $\Phi_{x}$ et $\Theta_{x}$, montre que $\left\|\Phi_{x}\right\|_{\Gamma}$ est égal à la norme $\left\|\Theta_{x}\right\|$ de $\Theta_{x}$ dans $L^{2}\left(G^{*} / \Gamma_{*}\right)$, prise au moyen de la mesure $d \dot{x}^{*}$; (19) donne alors $\|\Phi\|_{G}^{2}=\|\Theta\|_{Q}^{2}$ à condition de poser, pour toute solution $\Theta$ de $(22)$ :

$$
\|\Theta\|_{Q}^{2}=\int_{Q}|\Theta(z)|^{2} d \dot{z}
$$

Mais d'autre part, si on remplace $x^{*}$ par $x^{*}+\xi^{*}$ dans (21), on voit que, pour tout $x^{*} \in G^{*}$, la fonction $\xi^{*} \rightarrow \Phi^{*}\left(x^{*}+\xi^{*}\right)$ est la transformée de Fourier de

$$
\dot{x}->\Theta\left(x, x^{*}\right) \cdot\left\langle x, x^{*}\right\rangle
$$

en appliquant à ces fonctions le théorème de Plancherel, puis intégrant sur $G^{*} / \Gamma_{*}$, on obtient

$$
\|\Theta\|_{Q}^{2}=\int_{G^{*} / \Gamma_{*}}\left(\int_{\Gamma_{*}}\left|\Phi^{*}\left(x^{*}+\xi^{*}\right)\right|^{2} d \xi^{*}\right) d \dot{x}^{*}
$$


En vertu du théorème de Plancherel appliqué à $\Phi$ et $\Phi^{*}$, et des résultats obtenus plus haut, cela montre que le second membre n'est autre que $\left\|\Phi^{*}\right\|^{2}$ pris sur $G^{*}$ au moyen de la mesure $d x^{*}$ duale de $d x$; par conséquent, on a $d x^{*}=d \xi^{*} d \dot{x}^{*}$.

18. Nous désignerons par $H(G, \Gamma)$ l'espace de Hilbert des solutions $\Theta$ de (22), partout localement intégrables sur $G \times G^{*}$ et telles que $\|\Theta\|_{Q}<+\infty$, cet espace étant muni de la norme $\|\Theta\|_{Q}$. Il résulte de ce qui précède que l'application $\Phi \rightarrow \Theta$, définie par (20) quand $\Phi$ est continue à support compact, se prolonge par continuité à une application linéaire $Z$ de $L^{2}(G)$ dans $H(G, \Gamma)$ qui conserve la norme. En général, pour $\Phi \in L^{2}(G)$, le second membre de $(20)$ n'a pas de sens. Mais soit $\Phi \in L^{2}(G) \cap L^{1}(G)$; alors il y a une partie négligeable $N$ de $G / \Gamma$ telle que $\Phi_{x}$ appartienne à $L^{2}(\Gamma) \cap L^{1}(\Gamma)$ chaque fois que $\dot{x}$ n'est pas dans $N$. Soit $\Theta$ la fonction définie par (20) pour $\dot{x} \notin N$ et égale à 0 pour $\dot{x} \in N$; c'est une solution de (22), et $\Theta_{x}$ est la transformée de Fourier de $\Phi_{x}$ chaque fois que $\dot{x} \notin N$; le théorème de Planeherel appliqué à ces fonctions, et combiné comme plus haut avec (19), donne alors de nouveau $\|\Phi\|_{G}^{2}=\|\Theta\|_{Q}^{2}$. De plus, si on écrit $\Phi$ comme limite, au sens de $L^{2}(G)$, d'une suite $\Phi_{n}$ de fonctions continues à support compact, et qu'on pose $\Theta_{n}=Z\left(\Phi_{n}\right)$, on voit exactement de même que $\Theta-\Theta_{n}$ a même norme que $\Phi-\Phi_{n}$; cette norme tend donc vers 0 pour $n \rightarrow+\infty$. Il s'ensuit qu'on a $\Theta=Z(\Phi)$.

Réciproquement, soit $\Theta \in H(G, \Gamma)$ tel que $|\Theta|$ soit intégrable sur $Q$; il est clair que les fonctions de cette nature sont partout denses dans $H(G, \Gamma)$. Il y aura une partie négligeable $N$ de $G / \Gamma$ telle que $\Theta_{x}$ appartienne à la fois à $L^{2}\left(G^{*} / \Gamma_{*}\right)$ et à $L^{1}\left(G^{*} / \Gamma_{*}\right)$ chaque fois que $\dot{x}$ n'est pas dans $N$. Remplaçons $\Theta$ par la fonction égale à $\Theta$ pour $\dot{x} \notin N$ et à 0 pour $\dot{x} \in N$. Cela fait, soit $\Phi$ la fonction sur $G$ définie par

$$
\Phi(x)=\int_{G * / \Gamma *} \Theta\left(x, x^{*}\right) d \dot{x}^{*}
$$

Les hypothèses faites sur $\Theta$ impliquent que $\Phi$ est partout localement intégrable; de plus, en substituant $x+\xi$ à $x$ dans (23), on voit que, pour tout $x, \Phi_{x}$ est la transformée de Fourier de $\Theta_{x}$. Le théorème de Plancherel appliqué à ces fonctions, combiné de nouveau avec (19), montre alors que $\|\Phi\|_{G}^{2}=\|\Theta\|_{Q}^{2}$, donc que $\Phi \in L^{2}(G)$. Si alors on écrit $\Phi$ comme limite, au sens de $L^{2}(G)$, d'une suite $\Phi_{n}$ de fonctions continues à support compact, on voit, en répétant le même raisonnement, que $\Theta$ est limite des $\Theta_{n}=Z\left(\Phi_{n}\right)$ dans $H(G, \Gamma)$. On a donc montré que l'application $\Theta \rightarrow \Phi$ définie par (23) pour les fonctions dont il s'agit se prolonge par continuité à une application linéaire de $H(G, \Gamma)$ sur $L^{2}(G)$, réciproque de $Z$. En particulier, $Z$ est un isomorphisme de $L^{2}(G)$ sur $H(G, \Gamma)$.

Considérons en particulier le cas où on a pris $\Phi \in S(G)$. Il est facile de vérifier (d'abord 
dans le cas d'un groupe " élémentaire ", puis en passant de là au cas général au moyen des définitions rappelées plus haut au $\left.\mathrm{n}^{\circ} 11\right)$ qu'alors $x \rightarrow \Phi_{x}$ est une application continue de $G$ dans $S(\Gamma)$. Il résulte immédiatement de là que le second membre de $(20)$ est " uniformément convergent " et définit une solution continue $\Theta$ de (22), qui dans ces conditions, d'après ce qu'on a dit plus haut, n'est autre que $Z(\Phi)$. Comme de plus la transformation de Fourier relative à $\Gamma$ détermine un isomorphisme topologique de $S(\Gamma)$ sur $S\left(G^{*} / \Gamma_{*}\right)$, il s'ensuit que $x \rightarrow \Theta_{x}$ est une application continue de $G$ dans $S\left(G^{*} / \Gamma_{*}\right)$, donc que le second membre de (23) définit une fonction continue sur $G$; comme celle-ci, d'après ce qui précède, doit coïncider avec $\Phi$ au sens de $L^{2}(G)$, elle n'est donc autre que $\Phi$. Autrement dit, pour $\Phi \in \mathcal{S}(G)$, on peut prendre pour $\Theta=Z(\Phi)$ une fonction continue, et alors les formules (20), (23) sont valables partout (et non pas seulement presque partout).

19. Au moyen de l'isomorphisme $Z$ de $L^{2}(G)$ sur $H(G, \Gamma)$, on peut transporter à $H(G, \Gamma)$ les groupes d'opérateurs unitaires précédemment définis dans $L^{2}(G)$; par abus de notation, nous écrirons le plus souvent ceux-ci comme si on avait identifié $H(G, \Gamma)$ avec $L^{2}(G)$ au moyen de $Z$. Par exemple, nous écrirons $U(w)$ au lieu de $Z \cdot U(w) \cdot Z^{-1}$; on vérifie immédiatement que cet opérateur est défini par la formule

$$
U(w) \Theta(z)=\Theta(z+w) F(z, w)
$$

De même, on notera $\mathbf{A}(G)$ le groupe formé par les opérateurs $t \cdot U(w)$ dans $H(G, \Gamma)$, pour $t \in T$, et $\mathbf{B}_{0}(G)$ le normalisateur de $\mathbf{A}(G)$ dans le groupe des automorphismes de $H(G, \Gamma)$. Bien entendu, s'il résulte de ce qui précède que l'opérateur $U(w)$ donné par (24) transforme toute solution de (22) en une solution de (22), ce fait est aussi évident a priori.

On conviendra maintenant de désigner par $B_{0}(G, \Gamma)$ le sous-groupe de $B_{0}(G)$ formé par les éléments $s=(\sigma, f)$ de $B_{0}(G)$ tels que $f$ prenne la valeur 1 en tout élément de $\Gamma \times \Gamma_{*}$ et que $\sigma$ induise sur $\Gamma \times \Gamma_{*}$ un automorphisme de $\Gamma \times \Gamma_{*}$. Pour tout $s=(\sigma, f)$ dans $B_{0}(G, \Gamma)$, on définira un opérateur $\mathbf{r}_{\Gamma}(s)$ dans $H(G, \Gamma)$ par la formule

$$
\mathbf{r}_{\Gamma}(s) \Theta(z)=\Theta(z \sigma) f(z),
$$

dont on vérifie immédiatement qu'il transforme toute solution $\Theta$ de (22) en une solution de la même équation. Il est alors évident que $\mathbf{r}_{\Gamma}$ est une représentation de $B_{0}(G, \Gamma)$ par des opérateurs unitaires. De plus, au moyen de (24), de (25), et de la formule (6) du $n^{\circ} 5$, on vérifie aussitôt qu'on a, pour tout $w$ :

$$
U(w) \mathbf{r}_{\Gamma}(s)=f(w) \cdot \mathbf{r}_{\Gamma}(s) U(w \sigma),
$$

ce qui montre que $\mathbf{r}_{\Gamma}(s)$ appartient à $\mathbf{B}_{0}(G)$ et que sa projection canonique sur $B_{0}(G)$ est $s$; en d'autres termes, $\mathbf{r}_{\Gamma}$ est un " relèvement " de $B_{0}(G, \Gamma)$ à $\mathbf{B}_{0}(G)$. On notera $\mathbf{B}_{0}(G, \Gamma)$ l'image 
de $B_{0}(G, \Gamma)$ par $\mathbf{r}_{\Gamma}$, et aussi, par abus de notations, le groupe déduit de celui-là par transport à $L^{2}(G)$ au moyen de $Z$; de même, on écrira $\mathbf{r}_{\Gamma}$ au lieu de $Z^{-1} \mathbf{r}_{\Gamma} Z$.

Tout ce qui précède reste valable, mais devient à peu près trivial, lorsqu'on prend $\Gamma=\{0\}$, d'où $\Gamma_{*}=G^{*}$ (resp. $\Gamma=G, \Gamma_{*}=\{0\}$ ). Alors $H(G, \Gamma)$ s'identifie d'une manière évidente à $L^{2}(G)$ (resp. à $L^{2}\left(G^{*}\right)$ ), et $Z$ à l'identité (resp. à la transformation de Fourier). Le groupe $B_{0}(G, \Gamma)$ est ici (avec les notations du $\mathrm{n}^{\circ} 6$ ) le groupe des automorphis de la forme $d_{0}(\alpha) t_{0}(f)$ (resp. $d_{0}(\alpha) t_{0}^{\prime}\left(f^{\prime}\right)$ ). Dans le cas $\Gamma=\{0\}$, le relèvement $\mathbf{r}_{\Gamma}$ est celui qui, à $d_{0}(\alpha) t_{0}(f)$, fait correspondre $d_{0}(\alpha) t_{0}(f)$; dans le cas $\Gamma=G$, c'est le relèvement qui se déduit de celui-là par dualité.

Théorìme 4. Pour toute fonction $\Phi \in \boldsymbol{S}(G)$ et tout $\mathbf{s} \in \mathbf{B}_{0}(G, \Gamma)$, on a

$$
\int_{\Gamma} \Phi(\xi) d \xi=\int_{\Gamma} \mathbf{s} \Phi(\xi) d \xi
$$

En effet, d'après les résultats des $\mathrm{n}^{\mathrm{os}} 11-12$, quand $\Phi$ est dans $S(G)$, il en est de même de $\Phi^{\prime}=\mathbf{s} \Phi$; alors, d'après le $\mathrm{n}^{\circ} 18$, les fonctions $\Theta=Z(\Phi)$ et $\Theta^{\prime}=\mathbf{s} \Theta=Z\left(\Phi^{\prime}\right)$ sont continues et s'expriment en tout point par la formule (20). Mais, si l'on écrit $\mathrm{s}=\mathbf{r}_{\Gamma}(s)$ avec $s \in B_{\mathbf{0}}(G, \Gamma)$, $\Theta^{\prime}$ et $\Theta$ doivent aussi satisfaire à (25) au sens de $H(G, \Gamma)$, done aussi en tout point puisqu'elles sont continues. Pour $z=0$, (25) donne $\Theta^{\prime}(0)=\Theta(0)$; en exprimant les deux membres au moyen de $\Phi^{\prime}, \Phi$ par la formule (20), on obtient le résultat annoncé.

Corolla I r. Pour toute fonction $\Phi \in S(G)$, la formule

$$
F(\mathrm{~s})=\int_{\Gamma} \mathbf{s} \Phi(\xi) d \xi \quad\left(\mathrm{s} \in \mathbf{B}_{0}(G)\right)
$$

définit sur le groupe $\mathbf{B}_{\mathbf{0}}(G)$ une fonction invariante par les translations à gauche de $\mathbf{B}_{\mathbf{0}}(G, \Gamma)$.

D'après les nos $11-12$, $\mathrm{s} \Phi$ appartient à $S(G)$ pour tout $\mathrm{s} \in \mathbf{B}_{\mathbf{0}}(G)$; d'après ce qu'on a vu au $n^{\circ} 18$, cela implique en particulier que s $\Phi$ induit sur $\Gamma$ une fonction qui appartient à $\mathcal{S}(\Gamma)$, done à $L^{1}(\Gamma)$. Le corollaire résulte alors immédiatement du théorème 4.

Comme on le verra par la suite, ce corollaire fournit un des plus puissants moyens dont on dispose pour la construction de fonctions automorphes.

20. Au $\mathrm{n}^{\circ} 13$, on a défini un certain relèvement $\mathrm{r}_{\mathbf{0}}$ de $\Omega_{\mathbf{0}}(G)$ dans $\mathbf{B}_{\mathbf{0}}(G)$. On désignera maintenant par $\Omega_{0}(G, \Gamma)$ l'ensemble des éléments $s=(\sigma, f)$ de $B_{0}(G, \Gamma)$ tels que $\gamma(s)$ soit un isomorphisme de $G^{*} \operatorname{sur} G$ et induise sur $\Gamma_{*}$ un isomorphisme de $\Gamma_{*}$ sur $\Gamma$; cet ensemble est contenu dans $\Omega_{0}(G)$. Nous allons montrer que, sur $\Omega_{0}(G, \Gamma), \mathbf{r}_{\Gamma}$ coïncide avec $\mathbf{r}_{0}$. Pour 
cela, on observera que, dans la formule (8) de la proposition $1, \mathrm{n}^{0} 7$, si $s$ appartient à $\Omega_{0}(G, \Gamma)$, les trois facteurs $d u$ second membre appartiennent à $B_{0}(G, \Gamma)$. Il suffit done de vérifier que $\mathbf{r}_{\Gamma}\left(t_{0}(f)\right)=\mathbf{t}_{\mathbf{0}}(f)$ quand $t_{0}(f) \in B_{0}(G, \Gamma)$, et que $\mathbf{r}_{\Gamma}\left(d_{0}^{\prime}(\gamma)\right)=\mathbf{d}_{\mathbf{0}}^{\prime}(\gamma)$ quand $d_{0}^{\prime}(\gamma) \in B_{0}(G, \Gamma)$. Pour $t_{0}(f)$, c'est immédiat. Pour $d_{0}^{\prime}(\gamma)$, on voit immédiatement, en faisant usage de (21), que les deux opérateurs en question ne différent l'un de l'autre que par un facteur réel $>0$; comme ils sont unitaires, ils sont donc égaux. De plus, en écrivant que le facteur par lequel ils diffèrent a la valeur 1 , on trouve ce qui suit : soit $\gamma$ un isomorphisme de $\left(G^{*}, \Gamma_{*}\right)$ sur $(G, \Gamma)$, c'est-à-dire un isomorphisme de $G^{*}$ sur $G$ induisant sur $\Gamma_{*}$ un isomorphisme de $\Gamma_{*}$ sur $\Gamma$; alors ce dernier isomorphisme, et l'isomorphisme de $G^{*} / \Gamma_{*}$ sur $G / \Gamma$ qui se déduit de $\gamma$ par passage au quotient, ont même module, égal à $|\gamma|^{\frac{1}{2}}$; cela donne en particulier $|\gamma|=1$ lorsque $\Gamma$ et $\Gamma_{*}$ sont tous deux discrets, ou lorsqu'ils sont tous deux compacts.

On arrive directement au même résultat sur $\mathbf{r}_{\Gamma}$ en transportant à $L^{2}(G)$, au moyen des formules (20) pour $Z$ et (23) pour $Z^{-1}$, l'opérateur $\mathbf{r}_{\Gamma}$ défini par (25), dans le cas où $s \in \Omega_{0}(G, \Gamma)$; on obtient alors pour $\mathbf{r}_{\Gamma}$ une expression qui coïncide avec celle donnée par (16) pour $\mathbf{r}_{0}$, à un facteur positif près qui est alors nécessairement égal à $\mathbf{l}$ (ce qui a les mêmes conséquences que plus haut).

Mentionnons aussi que, lorsque $\Gamma$ et $\Gamma_{*}$ sont discrets et qu'on prend pour $\gamma$ un isomorphisme de $\left(G^{*}, \Gamma_{*}\right)$ sur $(G, \Gamma)$, puis qu'on prend $s=d_{0}^{\prime}(\gamma)$, donc $\mathbf{r}_{\Gamma}(s)=\mathbf{d}_{0}^{\prime}(\gamma)$, le théorème 4 se réduit à la formule classique de Poisson, dont il constitue done une généralisation.

THÉOR ̀̀ 5. Soit f un caractère $d u$ second degré de $G$, prenant la valeur 1 en tous les éléments d'un sous-groupe fermé $\Gamma$ de $G$; soient $G^{*}$ le dual de $G, \Gamma_{*}$ le sous-groupe de $G^{*}$ correspondant à $\Gamma$, et supposons que le morphisme symétrique $\varrho$ de $G$ dans $G^{*}$ associé à $f$ soit un isomorphisme de $(G, \Gamma)$ sur $\left(G^{*}, \Gamma_{*}\right)$. Alors $\gamma(f)=1$.

Il suffit pour le voir de se reporter à la démonstration du théorème 2; on y a obtenu $\gamma(f)$ comme étant le facteur par lequel diffèrent les deux membres de (9) après qu'on les a relevés à $\mathbf{B}_{0}(G)$ au moyen de $\mathbf{d}_{0}^{\prime}$ et $\mathbf{t}_{0}$. Mais, avec les hypothèses du théorème 5 , tous les facteurs de (9) sont dans $B_{0}(G, \Gamma)$; par suite, comme on vient de le voir, leurs relèvements par $d_{0}^{\prime}$ et $t_{0}$ coïncident avec leurs relèvements par $\mathbf{r}_{\Gamma}$. Comme $\mathbf{r}_{\Gamma}$ est une représentation, le résultat s'ensuit. On pourrait aussi (ce qui d'ailleurs revient au même) faire usage du théorème 3 , en y choisissant pour $s, s^{\prime}$ des éléments de $B_{0}(G, \Gamma)$ tels que $f_{0}=f$.

, Comme on le verra au Chapitre II, le théorème 5, appliqué à un groupe de type adélique (cas $(c)$ du début du $n^{\circ} 16$ ), contient la loi de réciprocité quadratique. Un cas particulier plus banal, mais utile, est le suivant : 
Corollair E. Soit $f$ le caractère $d u$ second degré donné par $f\left(x, x^{*}\right)=\left\langle x, x^{*}\right\rangle$ sur $G \times G^{*}$. Alors $f$ est non dégénéré, et $\gamma(f)=1$.

Le dual de $G \times G^{*}$ s'identifie d'une manière évidente à $G^{*} \times G$; le morphisme associé à $f$ est celui qui échange les deux facteurs de $G \times G^{*}$; donc $f$ est non dégénéré. En substituant respectivement $G \times G^{*}, G^{*} \times G, G \times\{0\}$ et $\{0\} \times G$ à $G, G^{*}, \Gamma$ et $\Gamma_{*}$ dans le théorème 5 , on obtient le résultat annoncé.

21. En vue d'applications ultérieures, ajoutons quelques remarques sur le cas où $\Gamma$ et $\Gamma_{*}$ sont compacts, ou encore (ce qui revient au même) où $\Gamma$ est compact et ouvert dans $G$. On choisira $d x$ et $d \xi$ de manière que la mesure de $\Gamma$ (pour $d x$ et pour $d \xi$ ) soit 1 . Alors $\Gamma_{*}$ a la mesure 1 pour $d x^{*}$ et pour $d \xi^{*}$.

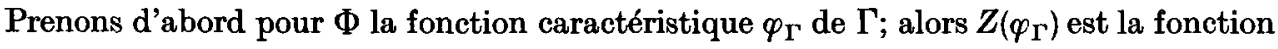
caractéristique de $\Gamma \times \Gamma_{*}$; d'après (25), elle est invariante par $\mathbf{B}_{0}(G, \Gamma)$; il en est donc de même, par définition, de $\Phi=\varphi_{\Gamma}$.

Prenons ensuite pour $\Phi$ la fonction caractéristique d'une classe suivant $\Gamma$, ou, ce qui revient au même, posons $\Phi(x)=\varphi_{\Gamma}(x-a)$; on voit aussitôt que $\Theta$ est alors la fonction $\varphi_{\Gamma}(x-a) \varphi_{\Gamma_{*}}\left(x^{*}\right)$, où $\varphi_{\Gamma_{*}}$ est la fonction caractéristique de $\Gamma_{*}$. Soit $s=(\sigma, f)$ un élément de $B_{0}(G, \Gamma)$, avec $\sigma=\left(\begin{array}{ll}\alpha & \beta \\ \gamma & \delta\end{array}\right)$. Pour que $\Theta$, et par conséquent $\Phi$, soient invariantes $\operatorname{par} \mathbf{r}_{\Gamma}(s)$, il faut et il suffit que l'on ait $a(\alpha-1) \in \Gamma, a \beta \in \Gamma_{*}$, et $f(a, 0)=1$; c'est ce qu'on voit immédiatement au moyen de (25).

Soit alors $\Gamma^{\prime}$ un sous-groupe ouvert compact de $G$, contenant $\Gamma$. Alors, avec les notations du $n^{0} 11$, l'ensemble $S\left(\Gamma^{\prime}, \Gamma\right)$ se compose des combinaisons linéaires à coefficients constants des fonctions $\varphi_{\Gamma}(x-a)$ pour $a \in \Gamma^{\prime}$; c'est un espace vectoriel, sur le corps des complexes, de dimension égale à l'indice (nécessairement fini) de $\Gamma$ dans $\Gamma^{\prime}$. Soit $s=(\sigma, f)$, comme ci-dessus, un élément de $B_{0}(G, \Gamma)$. Alors, pour que toute fonction de $S\left(\Gamma^{\prime}, \Gamma\right)$ soit invariante par $\mathbf{r}_{\Gamma}(s)$, il faut et il suffit, d'après ce qui précède, que $f$ prenne la valeur 1 sur $\Gamma^{\prime} \times \Gamma_{*}$, que $\Gamma^{\prime} \cdot(\alpha-1) \subset \Gamma$, et que $\Gamma^{\prime} \cdot \beta \subset \Gamma_{*}$. Il revient au même de dire que $f$ doit prendre la valeur 1 sur $\Gamma^{\prime} \times \Gamma_{*}$, que $\sigma$ doit induire un automorphisme sur $\Gamma^{\prime} \times \Gamma_{*}$, et que $\sigma$ détermine, par passage au quotient, l'automorphisme identique sur $\left(\Gamma^{\prime} \times \Gamma_{*}\right) /\left(\Gamma \times \Gamma_{*}\right)$.

22. Enfin, on notera quelques propriétés "fonctorielles " évidentes, pour le cas où on considère un produit $G=G_{1} \times G_{2}$, dont on peut écrire le dual sous la forme $G^{*}=G_{1}^{*} \times G_{2}^{*}$; on identifiera d'une manière évidente $G \times G^{*}$ avec $\left(G_{1} \times G_{1}^{*}\right) \times\left(G_{2} \times G_{2}^{*}\right)$. Alors, si $\sigma_{1}$, $\sigma_{2}$ sont des automorphismes de $G_{1} \times G_{1}^{*}$ et de $G_{2} \times G_{2}^{*}$, respectivement, on en déduit d'une manière évidente un automorphisme de $G \times G^{*}$ qu'on notera $\left(\sigma_{1}, \sigma_{2}\right)$; si $f_{1}, f_{2}$ sont des caractères du 
second degré sur $G_{1} \times G_{1}^{*}$ et sur $G_{2} \times G_{2}^{*}$, on en déduit un caractère du second degré $f=f_{1} \otimes f_{2}$ sur $G \times G^{*}$, défini par $f\left(z_{1}, z_{2}\right)=f_{1}\left(z_{1}\right) f_{2}\left(z_{2}\right)$; si $s_{1}=\left(\sigma_{1}, f_{1}\right), s_{2}=\left(\sigma_{2}, f_{2}\right)$ sont des éléments de $B_{0}\left(G_{1}\right)$ et de $B_{0}\left(G_{2}\right)$, respectivement, $\left(\left(\sigma_{1}, \sigma_{2}\right), f_{1} \otimes f_{2}\right)$ sera un élément de $B_{0}(G)$, qu'on notera $s_{1} \otimes s_{2}$. Chaque fois que ce sera commode, on identifiera $B_{0}\left(G_{1}\right) \times B_{0}\left(G_{2}\right)$ avec le groupe des éléments $s_{1} \otimes s_{2}$ de $B_{0}(G)$, et on identifiera $B_{0}\left(G_{1}\right), B_{0}\left(G_{2}\right)$ avec les facteurs de ce groupe.

On peut d'autre part considérer $L^{2}(G)$ comme " produit tensoriel hilbertien " de $L^{2}\left(G_{1}\right)$ et $L^{2}\left(G_{2}\right)$; alors, si $\mathrm{s}_{1}$, $\mathrm{s}_{2}$ sont des automorphismes de $L^{2}\left(G_{1}\right)$ et $\operatorname{de} L^{2}\left(G_{2}\right)$, respectivement, on en déduit d'une manière évidente un automorphisme $\mathrm{s}_{1} \otimes \mathrm{s}_{2}$ de $L^{2}(G)$. Pour chacun des groupes $G_{1}, G_{2}, G$, on pourra définir, comme au $\mathrm{n}^{\circ} 4$, des opérateurs $U\left(w_{1}\right), U\left(w_{2}\right)$, $U(w)$, qui sont respectivement des automorphismes de $L^{2}\left(G_{1}\right), L^{2}\left(G_{2}\right), L^{2}(G)$; et on aura, pour $w=\left(w_{1}, w_{2}\right)$ :

$$
U(w)=U\left(w_{1}\right) \otimes U\left(w_{2}\right)
$$

Il est évident alors que $\left(\mathbf{s}_{1}, \mathbf{s}_{2}\right) \rightarrow \mathrm{s}_{1} \otimes \mathbf{s}_{2}$ détermine sur $\mathbf{B}_{0}\left(G_{1}\right) \times \mathbf{B}_{0}\left(G_{2}\right)$ un homomorphisme de ce groupe dans $\mathbf{B}_{0}(G)$, compatible avec les projections canoniques de $\mathbf{B}_{0}\left(G_{1}\right)$ sur $B_{0}\left(G_{1}\right)$, de $\mathbf{B}_{0}\left(G_{2}\right)$ sur $B_{0}\left(G_{2}\right)$ et de $\mathbf{B}_{0}(G)$ sur $B_{0}(G)$; le noyau de cet homomorphisme est formé par les éléments $\left(\mathbf{t}, \mathbf{t}^{-1}\right)$ du centre; et il induit sur $\mathbf{B}_{0}\left(G_{1}\right)$ et $\mathbf{B}_{0}\left(G_{2}\right)$ des monomorphismes de ces groupes dans $\mathbf{B}_{0}(G)$, au moyen desquels on pourra, chaque fois que ce sera commode, les identifier avec leurs images dans ce dernier groupe.

\section{Application à la loi de réciprocité quadratique}

23. Pour mettre en harmonie autant que possible nos notations algébriques avec celles du Chapitre I, nous conviendrons, chaque fois que $X$ sera un espace vectoriel (toujours supposé de dimension finie) sur un corps $k$, de désigner son dual par $X^{*}$, et de noter $\left[x, x^{*}\right]$, pour $x \in X, x^{*} \in X^{*}$, la valeur en $x$ de la forme linéaire sur $X$ qui correspond à $x^{*}$; nous identifierons $X$ avec son bidual $\left(X^{*}\right)^{*}$ au moyen de la formule

$$
\left[x, x^{*}\right]=\left[x^{*}, x\right]
$$

et, chaque fois que $\alpha$ sera une application linéaire (dite aussi "morphisme ") d'un espace $X$ dans un espace $Y$, nous noterons $\alpha^{*}$ sa transposée, c'est-à-dire l'application linéaire de $Y^{*}$ dans $X^{*}$ définie, pour $x \in X, y^{*} \in Y^{*}$, par la formule

$$
\left[x \alpha, y^{*}\right]=\left[x, y^{*} \alpha^{*}\right]
$$

Toute forme bilinéaire sur $X \times Y$ s'écrit $[x, y \alpha]$, où $\alpha$ est un morphisme de $Y$ dans $X^{*}$; pour $X=Y$, on dira que $\alpha$ est symétrique si $[x, y \alpha]$ est symétrique en $x$ et $y$, donc si $\alpha=\alpha^{*}$. 
Si $f$ est une forme quadratique sur $X$, on a, quels que soient $x \in X, y \in X$ :

$$
f(x+y)-f(x)-f(y)=[x, y \varrho]
$$

où $\varrho$ est un morphisme symétrique de $X$ dans $X^{*}$; on dira alors que $f$ et $\varrho$ sont associés l'une à l'autre; $f$ sera dite non dégénérée si $\varrho$ est un isomorphisme de $X$ sur $X^{*}$, et additive si $\varrho=0$. Il n'y a de forme quadratique additive, autre que 0 , que si $k$ est de caractéristique 2 . Si $k$ n'est pas de caractéristique 2, tout morphisme symétrique $\varrho$ de $X$ dans $X^{*}$ est associé à une forme quadratique $f$ et à une seule, à savoir celle qui est donnée par $f(x)=\left[x, 2^{-1} x \varrho\right]$. En tout cas, on a $2 f(x)=[x, x \varrho]$. On conviendra de désigner par $Q(X)$ l'espace vectoriel des formes quadratiques sur $X$, et par $Q_{a}(X)$ le sous-espace de $Q(X)$ constitué par les formes additives.

24. Soit d'abord $k$ un corps local; nous entendrons par là un corps commutatif localement compact, non discret; il est donc, soit isomorphe à $\mathbf{R}$ ou à $\mathbf{C}$, soit à valuation discrète; et, dans ce dernier cas, c'est une extension finie, soit d'un corps $\mathbf{Q}_{p}$ (complétion $p$-adique du corps $\mathbf{Q}$ des rationnels) s'il est de caractéristique 0 , soit du corps des séries formelles à une indéterminée sur le corps premier $\mathbf{F}_{p}$ s'il est de caractéristique $p$. Si $k$ est à valuation discrète, on notera $\mathrm{o}$ l'anneau des entiers de $k$, et $\pi$ un élément premier de 0 , c'est-à-dire un générateur de l'idéal premier $\mathfrak{p}$ de $\mathfrak{o}$; on notera $q$ le nombre d'éléments du corps fini $\mathfrak{o} / \mathfrak{p}$. On choisira une fois pour toutes un caractère $\chi$ du groupe additif de $k$, dont nous supposerons seulement qu'il n'est pas trivial (c'est-à-dire qu'il n'a pas la valeur constante 1); il est possible, comme on sait, de choisir $\chi$ d'une manière "canonique ", mais cela nous serait inutile. On sait que $\chi(x y)$ est alors un bicaractère de $k \times k$ qui met $k$ en dualité avec lui-même au sens de la théorie des groupes abéliens localement compacts. Plus généralement, soit $X$ un espace vectoriel (de dimension finie, comme toujours) sur $k$; soit $X^{*}$ son dual; $X$ et $X^{*}$ étant munis de la topologie évidente, on peut alors identifier $X^{*}$ avec le dual de $X$ au sens de la théorie des groupes abéliens localement compacts de manière à avoir, pour tout $x \in X$ et tout $x^{*} \in X^{*}$ :

$$
\left\langle x, x^{*}\right\rangle=\chi\left(\left[x, x^{*}\right]\right)
$$

Cette identification (qui dépend du choix de $\chi$ ) sera faite désormais une fois pour toutes, de sorte qu'on n'aura pas à distinguer entre le dual algébrique et le dual au sens du Chapitre I.

Si $f$ est une forme quadratique sur un espace $X$ sur $k, \chi \circ f$ est un caractère du second degré sur $X$ au sens du Chapitre $\mathrm{I}, \mathrm{n}^{\circ} 1$; le morphisme de $X$ dans $X^{*}$ associé à $\chi \circ f$ est celui-là même qui est associé à $f$; en particulier, pour que $\chi \circ f$ soit non dégénéré, il faut et 
il suffit que $f$ le soit, et, lorsqu'il en est ainsi, on peut appliquer à $\chi \circ f$ le théorème 2 du Chapitre $\mathrm{I}, \mathrm{n}^{\circ} 14$, ce qui définit un nombre $\gamma(\chi \circ f)$ de valeur absolue 1 . Pour abréger, on écrira $\gamma(f)$ au lieu de $\gamma(\chi \circ f)$, mais il ne faudra pas oublier qu'alors le symbole $\gamma(f)$ dépend du choix de $\chi$. En revanche, comme $|\gamma(f)|=1$, il ne dépend pas du choix des mesures de Haar dans les groupes qui interviennent dans sa définition, puisque, lorsqu'on change ces mesures, cela ne modifie les formules du théorème $2 \mathrm{du}$ Chapitre $\mathrm{I}, \mathrm{n}^{\circ} 14$, et de ses corollaires, que par des facteurs réels $>0$; cette remarque permet même, dans le calcul de $\gamma(f)$ au moyen de ces corollaires, d'abandonner la convention, faite au Chapitre I, de toujours prendre dans le dual $G^{*}$ d'un groupe $G$ la mesure duale de celle qu'on a choisie dans $G$.

25. La remarque ci-dessus montre en particulier que, si $f$ et $X$ sont comme plus haut et si $f^{\prime}=f \circ \alpha$, où $\alpha$ est un isomorphisme d'un espace $X^{\prime}$ sur $X$, on a $\gamma\left(f^{\prime}\right)=\gamma(f)$; en d'autres termes, $\gamma$ a même valeur pour deux formes " équivalentes " $f, f^{\prime}$. D'autre part, comme $\chi \circ(-f)$ est l'imaginaire conjugué de $\chi \circ f$, on a $\gamma(-f)=\gamma(f)^{-1}$. Si -1 est un carré dans $k$ (done en particulier si $k$ est de caractéristique 2), - $f$ est équivalente à $f$; dans ce cas, on a donc $\gamma(f)= \pm 1$ quelle que soit $f$.

Proposition 3. L'application $f \rightarrow \gamma(f)$ détermine un caractère du groupe de Witt du corps local $k$.

Comme on sait, une forme $f$ non dégénérée correspond à l'élément 0 du groupe de Witt (ce que nous exprimerons, pour abréger, en disant qu'elle est triviale) si elle est équivalente à la forme $\sum_{1}^{n} x_{i} x_{n+i}$ sur $k^{2 n}$ pour une valeur convenable de $n$; cela s'exprime aussi en disant que $f$ est équivalente à la forme $\left[x, x^{*}\right]$ sur $X \times X^{*}$ pour un certain choix de $X$. Mais alors le corollaire $\mathrm{du}$ théorème $5 \mathrm{du}$ Chapitre $\mathrm{I}, \mathrm{n}^{\circ} 20$, montre que $\gamma(f)=1$. D'autre part, soient $f_{1}, f_{2}$ des formes non dégénérées sur des espaces $X_{1}, X_{2}$; soit $f$ la forme donnée par $f\left(x_{1}, x_{2}\right)=$ $f_{1}\left(x_{1}\right)+f_{2}\left(x_{2}\right)$ sur la somme directe de $X_{1}$ et de $X_{2}$; elle est non dégénérée, et il est évident, d'après la définition de $\gamma$ dans le théorème 2 du Chapitre $\mathrm{I}, \mathrm{n}^{\circ} \mathbf{1 4}$, que $\gamma(f)=\gamma\left(f_{1}\right) \gamma\left(f_{2}\right)$. Cela démontre la proposition.

26. Nous allons maintenant aborder le calcul de $\gamma(f)$, tout au moins dans certains cas particuliers. On conviendra de noter $q_{m}$ la forme $q_{m}(x)=\sum_{1}^{m} x_{i}^{2}$ sur l'espace $k^{m}$; elle est non dégénérée chaque fois que $k$ n'est pas de caractéristique 2 .

Soit d'abord $k=\mathbf{R}$; alors toute forme quadratique non dégénérée est équivalente à une forme $q_{a}(x)-q_{b}(y)$ sur un espace $\mathbf{R}^{a} \times \mathbf{R}^{b} ;(a, b)$ s'appelle le type d'inertie de la forme; la proposition 3 montre que, si $f$ a le type d'inertie $(a, b), \gamma(f)=\gamma\left(q_{1}\right)^{a-b}$. Le caractère $\chi$ sur $\mathbf{R}$ est nécessairement de la forme $\chi(x)=e^{2 \pi i \lambda x}$, avec $\lambda$ réel non nul. Pour calculer $\gamma\left(q_{1}\right)$, on appliquera le corollaire 2 du th. $2, n^{\circ} 14$, du Chapitre $I$, en prenant par exemple 
$\Phi(x)=e^{-\pi x^{2}}$; un calcul élémentaire donne alors pour $\gamma\left(q_{1}\right)$ la valeur $e^{\pi i / 4}$ si $\lambda>0$ et $e^{-\pi i / 4}$ si $\lambda<0$. Par suite, dans tous les cas, $\gamma(f)$ est une racine huitième de l'unité. On notera qu'on a toujours aussi $\gamma\left(q_{4}\right)=-1$ pour la forme $q_{4}(x)$, norme du quaternion $x_{1}+x_{2} i+x_{3} j+x_{4} k$.

Soit maintenant $k=\mathrm{C}$; alors toute forme est équivalente à une forme $q_{m}$, et il suffit de déterminer $\gamma\left(q_{1}\right)$, ou encore $\gamma\left(c q_{1}\right)$ pour une valeur quelconque de $c$. Si $\chi_{0}$ désigne le caractère $e^{2 \pi i x}$ sur $\mathbf{R}$, le caractère $\chi$ sur $\mathbf{C}$ sera nécessairement de la forme $\chi(z)=\chi_{0}(\lambda z+\bar{\lambda} \bar{z})$, avec $\lambda \neq 0$; pour $f=\lambda^{-1} q_{1}$, on aura $\chi \circ f=\chi_{0} \circ f_{0}$, où $f_{0}$ est donnée par $f_{0}(z)=z^{2}+\bar{z}^{2}$. Sur l'espace de dimension 2 sur $\mathbf{R}$ qui est sous-jacent à $\mathbf{C}, f_{0}$ est une forme quadratique de type d'inertie $(1,1)$; d'après ce qui précède, on a donc $\gamma\left(\chi_{0} \circ f_{0}\right)=1$, ou en d'autres termes $\gamma(\chi \circ f)=1$, e'est-à-dire $\gamma(f)=1$. Il en est donc de même pour toute forme quadratique non dégénérée sur $\mathbf{C}$.

27. Prenons maintenant pour $k$ un corps local à valuation discrète. Si $X$ est un espace vectoriel sur $k$, un sous-groupe compact et ouvert de $X$ sera appelé un réseau si c'est un module sur D; il est clair que, si $L$ est un réseau dans $X, L_{*}$ en est un dans $X^{*}$. Si $L$ et $L^{\prime}$ sont deux réseaux dans $X$, et si $L \supset L^{\prime}$, on notera $S\left(L, L^{\prime}\right)$, comme au $n^{\circ} 21$ du Chapitre I, la famille des fonctions à support contenu dans $L$ et constantes sur les classes suivant $L^{\prime}$. La réunion des familles $S\left(L, L^{\prime}\right)$, pour tous les choix possibles de $L$ et $L^{\prime}$, n'est autre que $S(X)$ (cf. $\mathrm{n}^{\circ} 11 \mathrm{du}$ Chapitre I).

Soit $f$ une forme quadratique non dégénérée sur $X$, associée à l'isomorphisme symétrique $\varrho$ de $X$ sur $X^{*}$. Comme le noyau de $\chi$ est un sous-groupe ouvert de $k, \chi \circ f$ a la valeur constante 1 sur un voisinage de 0 dans $X$, donc sur tout réseau suffisamment petit dans $X$. Choisissons un réseau $L$ tel que $\chi \circ f$ soit égal à 1 sur $L$, ce qui entraîne que $\chi([y, x \varrho])=1$ quels que soient $x \in L, y \in L$ (la réciproque étant d'ailleurs vraie chaque fois que 2 est inversible dans $\mathfrak{D}$ ), et par suite $L_{\varrho} \subset L_{*}$; on posera $L^{\prime}=L_{*} \varrho^{-1}$, et on aura donc $L^{\prime} \supset L$. Si $\varphi_{L}$ est la fonction caractéristique de $L$, on a alors

$$
\left(\varphi_{L} *(\chi \circ f)\right)(x)=\int_{L} \chi(f(x-y)) d y=\chi(f(x)) \int_{L} \chi([y,-x \varrho]) d y
$$

L'intégrale du dernier membre a la valeur $m(L)=\int_{L} d y$, ou 0 , suivant que $x \varrho$ est ou non dans $L_{*}$. On a done

$$
\varphi_{L} *(\chi \circ f)=m(L) \varphi_{L^{\prime}} \cdot(\chi \circ f)
$$

Appliquons maintenant à $\chi \circ$ of et à $\Phi=\varphi_{L}$ le corollaire 2 du théorème 2, Chapitre $\mathrm{I}, \mathrm{n}^{\circ} 14$; il s'ensuit que $\gamma(f)$ ne diffère que par un facteur réel $>0$ de l'intégrale de $\chi \circ f$ sur $L^{\prime}$. Posons, pour tout réseau $M$ : 
si $M \supset L$, cela peut s'écrire

$$
g(f, M)=\int_{M} \chi(f(x)) d x
$$

$$
g(f, M)=\sum_{x \in M / L} \chi(f(x)) \int_{L} \chi([y,-x \varrho]) d y,
$$

ou encore, en posant $M^{\prime}=M \cap L^{\prime}$ et tenant compte de ce qui précède :

$$
g(f, M)=m(L) \sum_{x \in M^{\prime} / L} \chi(f(x))
$$

La somme du second membre est une somme de Gauss; de plus, le second membre est indépendant de $M$ pourvu que $M \supset L^{\prime}$, donc dès que $M$ est assez grand. En notant simplement $g(f)$ sa valeur pour $M$ assez grand, on a donc $g\left(f, L^{\prime}\right)=g(f)$, et par suite, d'après ce qu'on a vu plus haut

$$
\gamma(f)=g(f) /|g(f)|
$$

Cela permet, si l'on veut, d'appliquer au calcul de $\gamma(f)$ la théorie des sommes de Gauss.

28. Indépendamment de la théorie des sommes de Gauss, on a le résultat suivant, suffisant pour notre objet :

Proposition 4. Soit l'algèbre de quaternions sur le corps local $k$, et soit $z \rightarrow n(z)=z \bar{z}$ la norme dans f. Alors $\gamma(n)=-1$.

D'après ce qui précède, tout revient à montrer que $g(n, M)$ est réel et $<0$ quand $M$ est un réseau assez grand dans f. Il est bien connu que $z \rightarrow n(z)$ détermine un homomorphisme surjectif, à noyau compact, du groupe multiplicatif de sur celui de $k$; de plus, quel que soit l'entier $\nu$, l'ensemble $M_{v}$ des $z \in \mathfrak{l}$ tels que $n(z) \in \pi^{-\nu} \mathrm{D}$ est un idéal, donc un réseau, de $\mathfrak{l}$, aussi grand qu'on veut si on a pris $\nu$ assez grand. Notons $d x, d z$ des mesures de Haar sur les groupes additifs de $k$ et de $\mathfrak{l}$, la première étant prise par exemple telle que la mesure de $\mathfrak{o}$ soit 1. Suivant l'usage, pour $a \in k$, nous définissons $|a|$ comme étant le module de $x \rightarrow a x$ dans $k$; il s'ensuit que $|x|^{-1} d x$ est une mesure de Haar sur le groupe multiplicatif de $k$. Mais alors, pour $c \in \mathbb{f}$, le module de $z \rightarrow c z$ dans $\mathfrak{f}^{\mathfrak{f}}$ est $|n(c)|^{2}$, et par suite $|n(z)|^{-2} d z$ est une

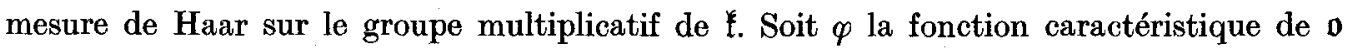
dans $k$; celle de $M_{v}$ dans $\mathfrak{l}^{\prime}$ est alors $\varphi\left(\pi^{v} n(z)\right)$, et on a done

$$
g\left(n, M_{v}\right)=\int \chi(n(z)) \varphi\left(\pi^{v} n(z)\right) d z
$$

Posons $\psi_{v}(x)=\chi(x) \varphi\left(\pi^{v} x\right)|x|^{2}$; nous pouvons écrire 12-642946 Acta mathematica. 111. Imprimé le 3 juin 1964. 


$$
g\left(n, M_{v}\right)=\int \psi_{\nu}(n(z)) \cdot|n(z)|^{-2} d z
$$

où l'intégrale est prise sur l'ensemble $\mathfrak{t}-\{0\}$, c'est-à-dire sur le groupe multiplicatif de $\mathfrak{k}$. Intégrant d'abord sur les classes suivant le groupe compact des $z$ tels que $n(z)=1$, classes sur lesquelles la fonction à intégrer est constante, puis sur le groupe quotient, on voit qu'on a

$$
g\left(n, M_{v}\right)=\lambda \int \psi_{\nu}(x) \cdot|x|^{-1} d x=\lambda \int \chi(x) \varphi\left(\pi^{v} x\right)|x| d x
$$

avec $\lambda$ réel $>0 ; \lambda$ est la mesure du noyau de $n$ pour la mesure quotient de $|n(z)|^{-2} d z$ par $|x|^{-1} d x$. Mais on a la relation évidente

$$
\varphi\left(\pi^{\nu} x\right) \cdot|x|=\sum_{\mu=-\nu}^{+\infty}\left|\pi^{\mu}\right| \cdot\left(\varphi\left(\pi^{-\mu} x\right)-\varphi\left(\pi^{-\mu-1} x\right)\right)
$$

comme on a $|\pi|=q^{-1}$, nous pouvons donc écrire:

$$
g\left(n, M_{\nu}\right)=\lambda \sum_{\mu=-\nu}^{+\infty} q^{-\mu}\left(\int_{\pi^{\mu_{0}}} \chi(x) d x-\int_{\pi^{\mu+1} 1_{0}} \chi(x) d x\right)
$$

Soit $m$ le plus petit des entiers $\mu$ tels que l'idéal $\mathfrak{p}^{\mu}=\pi^{\mu} \mathfrak{D}$ soit contenu dans le noyau du caractère $\chi$ de $k$; il est clair que l'intégrale de $\chi$ sur $\mathfrak{p}^{\mu}$ a la valeur $m\left(\mathfrak{p}^{\mu}\right)=q^{-\mu}$ lorsque $\chi$ induit la constante 1 sur $\mathfrak{p}^{\mu}$, donc pour $\mu \geqslant m$, et la valeur 0 dans le cas contraire. On obtient donc, pourvu que $v \geqslant 1-m$ :

$$
g\left(n, M_{v}\right)=-\lambda q^{1-2 m}\left(1+q^{-1}\right)^{-1}
$$

ce qui achève la démonstration.

On notera que, d'après le $n^{\circ} 26$, la proposition 4 est valable aussi pour $k=\mathbf{R}$, et trivialement valable pour $k=\mathbf{C}$ puisqu'il n'y a pas d'algèbre de quaternions sur $\mathbf{C}$. En ce sens, elle est donc valable pour tous les corps locaux.

On sait d'ailleurs que, sur tout corps local $k$ autre que $\mathbf{C}$, il n'y a que deux classes de formes quadratiques non dégénérées à 4 variables dont le discriminant soit un carré dans $k$; ces classes sont respectivement représentées par la forme $n$ étudiée ci-dessus, et par la forme "triviale " $x y+z t$. Les propositions 3 et 4 montrent que le symbole $\gamma(f)$ permet de distinguer entre ces classes de formes, puisqu'il a la valeur -1 pour la première et 1 pour la seconde. En particulier, si $k$ n'est pas de caractéristique 2, et si $a$ et $b$ sont des éléments non nuls de $k$, la forme $x^{2}-a y^{2}-b z^{2}+a b t^{2}$ appartient à l'une ou à l'autre des classes ci-dessus suivant que $a$ est ou non norme d'un élément de $k\left(b^{\frac{1}{2}}\right)$. On a donc

$$
\gamma\left(x^{2}-a y^{2}-b z^{2}+a b t^{2}\right)=(a / b)
$$


où $(a / b)$ désigne le symbole de restes normiques. Pour $b=-1$, cela donne, d'après la proposition $3, \gamma\left(q_{1}\right)^{2} \gamma\left(-a q_{1}\right)^{2}=(a /-1)$, ce qui s'écrit aussi $\gamma\left(a q_{1}\right)^{2}=(a /-1) \gamma\left(q_{1}\right)^{2}$. Comme toute forme non dégénérée peut s'écrire $\sum a_{i} x_{i}^{2}$ par le choix d'une base convenable, on en conclut, par une nouvelle application de la proposition 3 :

$$
\gamma(f)^{2}=(D /-1) \gamma\left(q_{1}\right)^{2 m}
$$

si $m$ est la dimension de l'espace où est définie $f$, et si $D$ est le discriminant de $f$ (pour un choix quelconque de la base, puisqu'un changement de base ne modifie $D$ que par un carré). On en conclut $\gamma(f)^{4}=\gamma\left(q_{4}\right)^{m}$, d'où $\gamma(f)^{8}=1$ puisque $q_{4}$ est non dégénérée de discriminant 1 . Ces résultats sont d'ailleurs triviaux si -1 est un carré dans $k$, puisqu'alors on a vu que $\gamma(f)= \pm 1$ quelle que soit $f$, et qu'alors $(D /-1)=1$ quel que soit $D$. Le dernier, $\gamma(f)^{8}=1$, est valable même en caractéristique 2 , puisqu'alors, comme on a vu, on a même $\gamma(f)= \pm 1$.

La loi de réciprocité quadratique va résulter de la combinaison des résultats ci-dessus avec l'application du théorème $5 \mathrm{du}$ Chapitre $\mathrm{I}, \mathrm{n}^{\circ} 20$, aux groupes de type adélique.

29. Soit $k$ un corps de nombres algébriques, ou bien un corps de fonctions algébriques de dimension 1 sur un corps fini. On désignera par $k_{v}$ les complétions de $k$, par $\mathfrak{b}_{v}$ l'anneau des entiers de $k_{v}$ chaque fois que $k_{v}$ est à valuation discrète, et par $A_{k}$ l'anneau des adèles de $k$. Soit $X_{k}$ un espace vectoriel (de dimension finie) sur $k$; on posera $X_{A}=X_{k} \otimes A_{k}$, et, pour tout $v, X_{v}=X_{k} \otimes k_{v}$. Si $X^{\circ}$ est une base de $X_{k}$ sur $k$, on notera $X_{v}^{\circ}$, chaque fois que $k_{v}$ est à valuation discrète, l'ensemble des points de $X_{v}$ dont les coordonnées par rapport à la base $X^{\circ}$ sont dans $\mathfrak{v}_{v}$; c'est un réseau dans $X_{v}$. On notera $S$ tout ensemble fini de complétions de $k$, contenant toutes celles qui sont isomorphes à $\mathbf{R}$ ou à $\mathbf{C}$. Dans ces conditions, $X_{A}$ est la réunion (et même, en tant qu'espace topologique, la limite inductive) des produits

$$
X_{S}^{\circ}=\prod_{v \in S} X_{v} \times \prod_{v \notin S} X_{v}^{\circ}
$$

Toute partie compacte de $X_{A}$ est contenue dans un ensemble de la forme $\Pi C_{v}$, où $C_{v}$ est, pour tout $v$, une partie compacte de $X_{v}$, et, pour presque tout $v$ (c'est-à-dire tout $v$ sauf un nombre fini d'entre eux), est égal à $X_{v}^{\circ}$. On en conclut que tout sous-groupe de $X_{A}$, engendré par un voisinage compact de 0 , est contenu dans un tel sous-groupe de la forme $H=\prod H_{v}$, où $H_{v}$ est égal à $X_{v}$ chaque fois que $k_{v}$ est isomorphe à $\mathbf{R}$ ou à $\mathbf{C}$, à un réseau dans $X_{v}$ chaque fois que $k_{v}$ est à valuation discrète, et à $X_{v}^{\circ}$ pour presque tout $v$. Supposons $H$ ainsi choisi; alors tout sous-groupe compact $H^{\prime}$ de $H$ tel que $H / H^{\prime}$ soit un groupe élémentaire (cf. Chapitre $\mathrm{I}, \mathrm{n}^{\circ} 11$ ) contient un sous-groupe analogue de la forme $H^{\prime}=\prod H_{v}^{\prime}$, où $H_{v}^{\prime}$ est égal à $\{0\}$ chaque fois que $k_{v}$ est isomorphe à $\mathbf{R}$ ou à $\mathbf{C}$, à un réseau de $X_{v}$ contenu dans $H_{v}$ chaque fois que $k_{v}$ est à valuation discrète, et à $X_{v}^{\circ}$ pour presque tout $v$. On déduit 
aisément de là la structure de $S\left(\mathrm{X}_{A}\right) ; \mathcal{S}\left(X_{A}\right)$ contient en tout cas toutes les fonctions de la forme $\left(x_{v}\right) \rightarrow \prod \Phi_{v}\left(x_{v}\right)$, où $\Phi_{v} \in S\left(X_{v}\right)$ pour tout $v$, et où, pour presque tout $v$, $\Phi_{v}$ est la fonction caractéristique de $X_{v}^{\circ}$; pour $k=Q$, ou pour $k$ de caractéristique $p \neq 0, S\left(X_{A}\right)$ est même l'ensemble des combinaisons linéaires finies, à coefficients constants, des fonctions ainsi obtenues (pour l'assertion correspondante lorsque $k$ est un corps de nombres algébriques autre que $Q$, voir plus loin, au $n^{\circ} 39$ ).

On choisira une fois pour toutes un caractère $\chi$ de $A_{k}$, non trivial, prenant la valeur 1 sur $k$; un tel caractère est nécessairement de la forme

$$
\chi(t)=\prod_{v} \chi_{v}\left(t_{v}\right) \quad\left(t=\left(t_{v}\right) \in A_{k}\right)
$$

où $\chi_{v}$ est, pour tout $v$, un caractère non trivial de $k_{v}$, et où, pour presque tout $v, \mathfrak{o}_{v}$ se correspond à lui-même par dualité lorsqu'on identifie $k_{v}$ avec son dual au moyen de $\chi_{v}$ de la manière qui a été expliquée au $\mathrm{n}^{\circ} 24$; cela implique que, pour presque tout $v, \chi_{v}$ induit la constante 1 sur $\mathfrak{D}_{v}$, de sorte que, dans l'expression ci-dessus de $\chi(t)$ par un produit infini, presque tous les facteurs ont la valeur 1 . On se servira de $\chi_{v}$ pour mettre $X_{v}$ en dualité avec $X_{v}^{*}$, de la manière expliquée au $\mathrm{n}^{\circ} 24$, chaque fois que $X_{k}$ est un espace vectoriel sur $k$ et que $X_{k}^{*}$ est son dual (au sens algébrique, conformément aux notations introduites au $\mathrm{n}^{\circ} 23$ ). Alors, si $X^{\circ}$ est une base de $X_{k}$ sur $k$ et $\left(X^{*}\right)^{\circ}$ une base de $X_{k}^{*}$ sur $k$ (par exemple la base duale de $\left.X^{\circ}\right)$, le réseau $\left(X_{v}^{\circ}\right)_{*}$ dans $X_{v}^{*}$ qui correspond par dualité à $X_{v}^{\circ}$ est $\left(X^{*}\right)_{v}^{\circ}$ pour presque tout $v$.

Il est bien connu aussi que, si $\chi$ est choisi comme ci-dessus, le bicaractère $\chi(x y)$ de $A_{k} \times A_{k}$ met $A_{k}$ en dualité avec lui-même de telle sorte que le sous-groupe $\operatorname{discret} k \operatorname{de} A_{k}$ se corresponde à lui-même par dualité. Alors, si $X_{k}$ est comme ci-dessus, on peut identifier $X_{A}^{*}$ avec le dual de $X_{A}$ au sens de la théorie des groupes abéliens localement compacts au moyen de la formule

$$
\left\langle x, x^{*}\right\rangle=\chi\left(\left[x, x^{*}\right]\right) \quad\left(x \in X_{A}, x^{*} \in X_{A}^{*}\right)
$$

où $\left[x, x^{*}\right]$ désigne l'extension évidente de la forme bilinéaire sur $X_{k} \times X_{k}^{*}$ définie au $\mathrm{n}^{\circ} 23$ à une application bilinéaire de $X_{A} \times X_{A}^{*}$ dans $A_{k}$. Dans cette dualité, les sous-groupes discrets $X_{k}, X_{k}^{*}$ de $X_{A}$ et $X_{A}^{*}$ se correspondent l'un à l'autre; autrement dit, on pourra prendre $G=X_{A}, G^{*}=X_{A}^{*}, \Gamma=X_{k}, \Gamma_{*}=X_{k}^{*}$ dans les $\mathrm{n}^{\text {os }} 16-20$ du Chapitre I.

30. Soit $f$ une forme quadratique non dégénérée sur $X_{k}$; elle détermine d'une manière évidente des formes quadratiques sur les espaces $X_{v}$, et une application $x \rightarrow f(x)$ de $X_{A}$ dans $A_{k}$, donc aussi des caractères du second degré, $\chi_{v} \circ f$ et $\chi \circ f$, sur les $X_{v}$ et sur $X_{A}$. Pour abréger, on écrira $\gamma_{v}(f), \gamma(f)$ au lieu de $\gamma\left(\gamma_{v} \circ f\right), \gamma(\chi \circ f)$. 
$\mathrm{P}_{\mathrm{Roposit}} \mathrm{Ton}$ 5. Soit $f$ une forme quadratique non dégénérée sur un espace vectoriel $X_{k}$ sur k. Alors on a

$$
\gamma(f)=\prod_{v} \gamma_{v}(f)=1
$$

Le théorème $5 \mathrm{du}$ Chapitre $\mathrm{I}, \mathrm{n}^{\circ} 20$, appliqué à $\chi \circ f, X_{A}$ et $X_{k}$, montre que $\gamma(f)=1$; il reste à faire voir que $\gamma(f)=\prod \gamma_{v}(f)$. Pour cela, soit $\Phi$ la fonction définie sur $X_{A}$ par $\Phi(x)=$ $\prod \Phi_{v}\left(x_{v}\right)$ pour $x=\left(x_{v}\right)$, avec $\Phi_{v} \in S\left(X_{v}\right)$ pour tout $v, \Phi_{v}$ étant pour presque tout $v$ la fonction caractéristique de $X_{v}^{\circ}$. On va appliquer le corollaire 2 du théorème 2 , Chapitre $1, \mathrm{n}^{\circ} 14$, d'une part à $\Phi$ et d'autre part aux fonctions $\Phi_{v}$. D'après les remarques faites à la fin du $\mathrm{n}^{\circ} 24$, le choix des mesures de Haar est indifférent ici; on prendra une mesure $m_{v}$ sur chaque $X_{v}$, de façon que $m_{v}\left(X_{v}^{\circ}\right)=1$ pour presque tout $v$; on en déduit une mesure produit sur chacun des ensembles $X_{S}^{\circ}$ définis par (29), et par suite une mesure sur $X_{A}$. D'après (27), $\mathrm{n}^{\circ} 27$, on voit immédiatement que, pour presque tout $v, \Phi_{v} *\left(\chi_{v} \circ f\right)=\Phi_{v}$, d'où $\gamma_{v}(f)=1$ par application du corollaire en question. En appliquant alors celui-ci à $X_{A}$, on écrira les intégrales sur $X_{A}$ comme limites des intégrales correspondantes sur les ensembles $X_{S}^{\circ}$. Cela conduit aussitôt au résultat annoncé.

Pour obtenir la loi de réciprocité quadratique, on appliquera la proposition ci-dessus à une forme non dégénérée à 4 variables dont le discriminant soit un carré dans $k$. Pour la forme obtenue comme norme d'une algèbre $\mathfrak{l}$ de quaternions sur $k$, cela donne la loi de réciprocité sous la forme due à Hasse, $\prod h_{v}(\mathfrak{f})=1$, où $h_{v}(\mathfrak{l})$ a la valeur 1 ou -1 suivant que $\otimes k_{v}$ est une algèbre de matrices ou une "vraie " algèbre de quaternions sur $k_{v}$. Pour la forme $x^{2}-a y^{2}-b z^{2}+a b t^{2}$ sur un corps $k$ de caractéristique autre que 2, cela donne la loi de réciprocité sous la forme de Hilbert, $\Pi(a / b)_{v}=1$.

Malgré les apparences, la démonstration de la loi de réciprocité quadratique exposée ci-dessus ne diffère pas substantiellement de la démonstration classique au moyen des fonctions thêta et des sommes de Gauss; on notera à cet égard le rôle essentiel joué par des fonctions thêta généralisées dans la démonstration du théorème 5 du Chapitre $\mathrm{I}$. D'autre part, désignons par $P(k, m)$ la propriété $\prod \gamma_{v}(f)=1$ pour les formes quadratiques non dégénérées $f$ sur les espaces $X_{k}$ de dimension $m$ sur le corps $k$. D'après la proposition 3, $P(k, m)$ résulte formellement de $P(k, 1)$ si $k$ n'est pas de caractéristique 2 , et de $P(k, 2)$ si $k$ est de caractéristique 2 . Soit de plus $k^{\prime}$ une extension séparable de $k$ de degré $d$; soit $\tau$ la trace, prise dans $k^{\prime}$ sur le corps de base $k$. On a alors $A_{k^{\prime}}=k^{\prime} \otimes A_{k}$; et, si $\chi$ est le caractère introduit ci-dessus pour $A_{k}, \chi^{\prime}=\chi \circ \tau$ est un caractère analogue pour $A_{k^{\prime}}$. Si $f^{\prime}$ est une forme quadratique non dégénérée dans un espace $X_{k^{\prime}}$ de dimension $m$ sur $k^{\prime}$, $\tau$ of' en est une dans l'espace sur $k$ sous-jacent à $X_{k^{\prime}}$. On en conclut aussitôt que $P(k, m d)$ implique $P\left(k^{\prime}, m\right)$. Il résulte de ces remarques que par exemple, dans le cas de caractéristique $0, P(k, m)$ est une conséquence formelle de $P(\mathbf{Q}, 1)$ quel que soit $m$ et quel que soit le corps de nombres algé- 
briques $k$. On notera aussi que la loi de réciprocité quadratique, sous la forme de Hasse ou sous la forme de Hilbert, est contenue dans $P(k, 4)$, de sorte que, formellement du moins, cette loi est un résultat moins fort que $P(k, 1)$ si $k$ n'est pas de caractéristique 2 , ou que $P(k, 2)$ si $k$ est de caractéristique 2 .

Nous terminerons ce chapitre par une question : peut-on donner de la loi générale de réciprocité une démonstration analogue à celle qu'on vient d'exposer pour la loi de réciprocité quadratique?

\section{Le groupe métaplectique (cas local et cas adélique)}

31. Comme au $\mathrm{n}^{\circ} 23$, soit d'abord $X$ un espace vectoriel (de dimension finie, comme toujours) sur un corps quelconque $k$; conservant les notations introduites au $\mathrm{n}^{\circ} 23$, nous aurons à considérer des automorphismes $z \rightarrow z \sigma$ de $X \times X^{*}$, qu'on notera, comme au Chapitre I (cf. $\mathrm{n}^{\circ} 3$ ), sous forme matricielle

$$
\sigma=\left(\begin{array}{ll}
\alpha & \beta \\
\gamma & \delta
\end{array}\right)
$$

Comme au $\mathrm{n}^{\circ} 3$, on posera $\quad \sigma^{\mathrm{I}}=\left(\begin{array}{rr}\delta^{*} & -\beta^{*} \\ -\gamma^{*} & \alpha^{*}\end{array}\right)$.

Sur $\left(X \times X^{*}\right) \times\left(X \times X^{*}\right)$, on considérera la forme bilinéaire

$$
B\left(z_{1}, z_{2}\right)=\left[x_{1}, x_{2}^{*}\right] \quad\left(z_{1}=\left(x_{1}, x_{1}^{*}\right), z_{2}=\left(x_{2}, x_{2}^{*}\right)\right),
$$

et on dira qu'un automorphisme $\sigma$ de $X \times X^{*}$ est symplectique s'il laisse invariante la forme bilinéaire $B\left(z_{1}, z_{2}\right)-B\left(z_{2}, z_{1}\right)$; pour qu'il en soit ainsi, il faut et il suffit qu'on ait $\sigma \sigma^{\mathrm{I}}=1$. Ces automorphismes forment un groupe qu'on appellera le groupe symplectique de $X$ et qu'on notera $S p(X)$.

Sur $X \times X^{*} \times k$, on mettra une structure de groupe au moyen de la loi de composition suivante (analogue à (4) du Chapitre I) :

$$
\left(z_{1}, t_{1}\right) \cdot\left(z_{2}, t_{2}\right)=\left(z_{1}+z_{2}, B\left(z_{1}, z_{2}\right)+t_{1}+t_{2}\right)
$$

on notera $\mathfrak{A}(X)$ le groupe ainsi défini. Soient alors $\sigma$ un automorphisme de $X \times X^{*}$ et $f$ une forme quadratique sur $X \times X^{*}$; pour que la formule

$$
(z, t) \rightarrow(z \sigma, f(z)+t) \quad\left(z \in X \times X^{*}, t \in k\right)
$$

définisse un automorphisme de $\mathfrak{A}(X)$, il faut et il suffit que $\sigma$ et $f$ satisfassent à la relation

$$
f\left(z_{1}+z_{2}\right)-f\left(z_{1}\right)-f\left(z_{2}\right)=B\left(z_{1} \sigma, z_{2} \sigma\right)-B\left(z_{1}, z_{2}\right),
$$


analogue à (6) du Chapitre I. Lorsqu'il en est ainsi, on désignera par $(\sigma, f)$ l'automorphisme de $\mathfrak{A}(X)$ défini par (31); le groupe formé par ces automorphismes sera appelé le groupe pseudosymplectique de $X$ et sera noté $P s(X)$. La loi de groupe dans $P s(X)$ est donnée par

$$
(\sigma, f) \cdot\left(\sigma^{\prime}, f^{\prime}\right)=\left(\sigma \sigma^{\prime}, f^{\prime \prime}\right),
$$

où $f^{\prime \prime}$ est la forme quadratique définie par

$$
f^{\prime \prime}(z)=f(z)+f^{\prime}(z \sigma)
$$

$\mathrm{Si}$ (32) est satisfaite, le second membre doit être symétrique en $z_{1}$ et $z_{2}$; pour qu'il en soit ainsi, il faut et il suffit que $\sigma$ soit symplectique; done $(\sigma, f) \rightarrow \sigma$ est un homomorphisme de $P s(X)$ dans $S p(X)$. Si $k$ n'est pas de caractéristique 2, (32) fait correspondre à tout $\sigma \in S p(X)$ une forme quadratique $f$ et une seule sur $X \times X^{*}$; par suite, dans ce cas, $(\sigma, f) \rightarrow \sigma$ est un isomorphisme de $P s(X)$ sur $S p(X)$, au moyen duquel on pourra, chaque fois que ce sera commode, identifier ces groupes l'un avec l'autre. Au contraire, quand $k$ est de caractéristique 2 , on voit, en prenant $z_{1}=z_{2}$ dans (32), que $\sigma$ laisse invariante la forme quadratique non dégénérée $B(z, z)$ sur $X \times X^{*}$; par suite, $(\sigma, f) \rightarrow \sigma$ est un homomorphisme de $P s(X)$ dans le groupe orthogonal $O(B)$ de cette forme dans $X \times X^{*}$; on vérifie aisément qu'il est surjectif; son noyau est formé par les éléments $(1, f)$ de $P s(X)$, où, d'après (32), on doit prendre pour $f$ toutes les formes quadratiques additives sur $X \times X^{*}$.

Si maintenant on part d'un espace vectoriel $X_{k}$ sur $k$, et qu'on note $X$ l'extension de $X_{k}$ à un « domaine universel " sur $k$, on peut appliquer ce qui précède, soit à $X_{k}$ et $k$, soit à $X$ et au domaine universel. Les groupes $S p(X), \mathfrak{A}(X), P s(X)$ sont alors des groupes algébriques définis sur $k$, et $S p\left(X_{k}\right), \mathfrak{A}\left(X_{k}\right), P s\left(X_{k}\right)$ ne sont autres que les groupes $S p(X)_{k}, \mathfrak{A}(X)_{k}$, $P s(X)_{k}$ formés des éléments de $S p(X), \mathfrak{A}(X), P s(X)$ qui sont rationnels sur $k$. On notera que $P s(X)$ est de dimension $m(2 m+1)$ si $m=\operatorname{dim}(X)$, quelle que soit la caractéristique de $k$; on peut dire (en un sens que le langage des schémas permet de préciser) que $P s(X)$ en caractéristique 2 est une dégénérescence du groupe symplectique en caractéristique 0 . Il est bien connu que le groupe symplectique, en toute caractéristique, est connexe, simplement connexe et semisimple; il en est donc de même de $P s(X)$ quand $k$ n'est pas de caractéristique 2 , mais ce n'est plus vrai en caractéristique 2 .

32. On a, pour le groupe $P s(X)$, des résultats entièrement analogues à ceux des $n^{\text {os }}$ 5,6 et $7 \mathrm{du}$ Chapitre I pour le groupe $B_{0}(G)$; nous allons les résumer rapidement. On définit un monomorphisme dans $P s(X)$ du groupe $\operatorname{Aut}(X)$ des automorphismes de $X$ en posant

$$
d(\alpha)=\left(\left(\begin{array}{ll}
\alpha & 0 \\
0 & \alpha^{*-1}
\end{array}\right), 0\right) .
$$


A. WEIL

On définit une application dans $P s(X)$ de l'ensemble $\operatorname{Is}\left(X^{*}, X\right)$ des isomorphismes de $X^{*} \operatorname{sur} X$ en posant

$$
d^{\prime}(\gamma)=\left(\left(\begin{array}{cc}
0 & -\gamma^{*-1} \\
\gamma & 0
\end{array}\right),\left[x,-x^{*}\right]\right)
$$

On définit un monomorphisme dans $P s(X)$ du groupe additif $Q(X)$ des formes quadratiques sur $X$ en posant

$$
t(f)=\left(\left(\begin{array}{ll}
1 & \varrho \\
0 & 1
\end{array}\right), f\right)
$$

où $\varrho$ est le morphisme symétrique de $X$ dans $X^{*}$ associé à la forme quadratique $f$ sur $X$. De même, si $f^{\prime}$ est une forme quadratique sur $X^{*}$, associée au morphisme $\varrho^{\prime}$ de $X^{*}$ dans $X$, on posera

$$
t^{\prime}\left(f^{\prime}\right)=\left(\left(\begin{array}{ll}
1 & 0 \\
g^{\prime} & 1
\end{array}\right), f^{\prime}\right)
$$

Entre les éléments de $P s(X)$ ainsi définis, on a des relations analogues à celles des $n^{\text {os }} 6-7$, et notamment

$$
d(\alpha)^{-1} t(f) d(\alpha)=t\left(f^{\alpha}\right), \quad d(\alpha) t^{\prime}\left(f^{\prime}\right) d(\alpha)^{-1}=t^{\prime}\left(f^{\prime \alpha^{*}}\right)
$$

où $f^{\alpha}$ est définie par $f^{\alpha}(x)=f\left(x \alpha^{-1}\right)$, et

$$
d^{\prime}(\gamma \alpha)=d^{\prime}(\gamma) d(\alpha), \quad d^{\prime}\left(\alpha^{*-1} \gamma\right)=d(\alpha) d^{\prime}(\gamma),
$$

pour $\alpha \in \operatorname{Aut}(X), \gamma \in \operatorname{Is}\left(X^{*}, X\right)$.

Pour $s=(\sigma, f), \sigma=\left(\begin{array}{ll}\alpha & \beta \\ \gamma & \delta\end{array}\right)$, posons $\gamma=\gamma(s)$; on conviendra de noter $\Omega(X)$ l'ensemble des $s \in P s(X)$ pour lesquels $\gamma(s)$ est inversible, c'est-à-dire pour lesquels c'est un isomorphisme de $X^{*}$ sur $X$. En répétant ici, avec des modifications évidentes, la démonstration de la proposition 1 du Chapitre $I, n^{\circ} 7$, on voit que tout $s \in \Omega(X)$ se met d'une manière et d'une seule sous la forme

$$
s=t\left(f_{1}\right) d^{\prime}(\gamma) t\left(f_{2}\right)
$$

où $f_{1}, f_{2}$ sont des formes quadratiques $\operatorname{sur} X$, et $\gamma \in \operatorname{Is}\left(X^{*}, X\right)$; on a $\gamma=\gamma(s)$. Plus précisément, $\Omega(X)$ est $k$-ouvert dans $P s(X)$ au sens de la topologie de Zariski, et (33) détermine un $k$ isomorphisme de variétés algébriques entre $\Omega(X)$ et $Q(X) \times \operatorname{Is}\left(X^{*}, X\right) \times Q(X)$; comme Is $\left(X^{*}, X\right)$ n'est pas vide, $\Omega(X)$ n'est pas vide. Le complémentaire de $\Omega(X)$ dans $P s(X)$ est d'ailleurs défini par det $\gamma(s)=0$, le déterminant étant pris pour un choix quelconque de bases dans $X$ et $X^{*}$; il s'ensuit que ce complémentaire est réunion de variétés de codimension 0 ou 1 dans $P s(X)$; c'est même une réunion de variétés de codimension 1 dans $P_{s}(X)$ chaque fois que $k$ n'est pas de caractéristique 2, puisqu'alors $P s(X)$ est isomorphe à $S p(X)$ quj 
est connexe, et que $\Omega(X)$ n'est pas vide. Si au contraire $k$ est de caractéristique 2 , on sait que le groupe orthogonal $O(B)$ comprend deux composantes connexes $O^{+}(B), O^{-}(B)$, formées respectivement par les éléments $\sigma=\left(\begin{array}{ll}\alpha & \beta \\ \gamma & \delta\end{array}\right)$ de $O(B)$ pour lesquels $\operatorname{tr}\left(\beta \gamma^{*}\right)$ a la valeur 0 ou 1; en désignant par $P s^{+}(X)$ et $P s^{-}(X)$ leurs images réciproques dans $P s(X)$, on vérifie sans difficulté que $\Omega(X)$ est contenu dans $P s^{+}(X)$ ou dans $P s^{-}(X)$ suivant que la dimension $m$ de $X$ est paire ou impaire. D'une manière plus précise, soient $s=(\sigma, f) \in P s(X)$ et $\sigma$ comme ci-dessus, et soit $r$ le rang de $\gamma$; alors on constate que $r \equiv \operatorname{tr}\left(\beta \gamma^{*}\right) \bmod$. 2, ce qui donne bien $\operatorname{tr}\left(\beta \gamma^{*}\right) \equiv m(\bmod .2)$ pour $r=m$, c'est-à-dire pour $\gamma$ inversible.

33. On va considérer maintenant le cas où $k$ est un corps local. Comme il a été expliqué au $\mathrm{n}^{\circ} 24 \mathrm{du}$ Chapitre II, on suppose donné, une fois pour toutes, un caractère non trivial $\chi$ de $k$, ce qui permet, chaque fois que $X$ est un espace vectoriel (de dimension finie) sur $k$, d'identifier le dual algébrique $X^{*}$ de $X$ avec le dual de $X$ au sens de la théorie des groupes abéliens localement compacts, donc d'appliquer à $G=X$ et $G^{*}=X^{*}$ la théorie exposée au Chapitre I.

La topologie donnée sur $k$ permet, d'une manière évidente, de munir les groupes $\mathfrak{U}(X), P s(X)$ du $\mathrm{n}^{\circ} 31$ de topologies qui en font des groupes localement compacts. L'application $(w, t) \rightarrow(w, \chi(t))$ de $\mathfrak{U}(X)$ dans le groupe $A(X)$ du $\mathrm{n}^{\circ} 4$ est évidemment un homomorphisme; il en est de même de l'application $\mu$ de $P s(X)$ dans le groupe $B_{0}(X)$ du n $n^{\circ} 5$ qui est définie par

$$
\mu((\sigma, f))=(\sigma, \chi \circ f)
$$

Le noyau de $\mu$ est formé des éléments de $P s(X)$ de la forme $(1, f)$, où $f$ est une forme quadratique sur $X$ telle que $\chi \circ f=1$; comme le morphisme symétrique de $X$ dans $X^{*}$ associé à $\chi \circ f$ est le même que celui qui est associé à $f$, cette dernière relation entraîne que $f$ est additive. Quand $k$ n'est pas de caractéristique $2, \mu$ est donc injectif; cela permet, dans ce cas, de simplifier les notations en identifiant $P s(X)$ avec son image dans $B_{0}(X)$; avec cette convention, les applications $d, d^{\prime}, t, t^{\prime} \mathrm{du} \mathrm{n}^{\circ} 32$ deviennent des restrictions, à des ensembles convenables, des applications $d_{0}, d_{0}^{\prime}, t_{0}, t_{0}^{\prime}$ du Chapitre $\mathrm{I}, \mathrm{n}^{\circ} 6$. Quand $k$ est de caractéristique 2 , il n'en est plus de même, et on peut dire seulement que les applications du $n^{\circ} 32$ sont «compatibles», en un sens évident, avec celles du $n^{\circ} 6$.

34. $\mathrm{Au} \mathrm{n}^{\circ} 10 \mathrm{du}$ Chapitre $\mathrm{I}$, on a défini, au moyen du théorème 1 , un groupe $\mathbf{B}_{0}(G)$ d'automorphismes de $L^{2}(G)$, ainsi que la projection canonique $\pi_{0}$ de ce groupe sur $B_{0}(G)$. Prenant maintenant $G=X$ comme au $\mathrm{n}^{\circ} 33$, nous appellerons groupe métaplectique de $X$, et nous désignerons par $M p(X)$, le sous-groupe de $P s(X) \times \mathbf{B}_{0}(X)$ formé des éléments $\mathbf{S}=(s, \mathbf{s})$ de ce produit tels que $\mu(s)=\pi_{0}(\mathbf{s})$. On notera $\pi$ l'application $(s, \mathbf{s}) \rightarrow s$ de $M p(X)$ 
dans $P s(X)$; comme $\pi_{0}$ est surjectif, $\pi$ est un homomorphisme surjectif de $M p(X)$ sur $P s(X)$; son noyau est $\{e\} \times \mathbf{T}$, où $e$ est l'élément neutre de $P s(X)$ et où $\mathbf{T}$, comme précédemment, désigne le groupe des opérateurs $\Phi \rightarrow t \Phi$, avec $t \in T$, c'est-à-dire $t t=1$; pour simplifier les notations, on identifiera ce noyau avec $\mathbf{T}$, ou même éventuellement avec $T$; il est contenu dans le centre de $M p(X)$.

L'application $(s, \mathrm{~s}) \rightarrow \mathrm{s}$ est une représentation de $M p(X)$ dans le groupe des automorphismes de $L^{2}(X)$; pour $\mathrm{S}=(s, \mathrm{~s}) \in M p(X)$ et $\Phi \in L^{2}(X)$, on écrira, chaque fois que ce sera commode, $\mathbf{S} \Phi$ au lieu de $\mathbf{S} \Phi$.

Les formules du Chapitre $I, \mathrm{n}^{\circ} 13$, jointes à celles du $\mathrm{n}^{\circ} 32$, permettent de définir comme suit des applications de Aut $(X)$, Is $\left(X^{*}, X\right)$ et $Q(X)$ dans $M p(X)$ :

$$
\begin{aligned}
& \mathbf{d}(\alpha)=\left(d(\alpha), \mathbf{d}_{0}(\alpha)\right) \quad(\alpha \in \operatorname{Aut}(X)), \\
& \mathbf{d}^{\prime}(\gamma)=\left(d^{\prime}(\gamma), \mathbf{d}_{0}^{\prime}(\gamma)\right) \quad\left(\gamma \in \operatorname{Is}\left(X^{*}, X\right)\right), \\
& \mathbf{t}(f)=\left(t(f), \mathbf{t}_{0}(\chi \circ f)\right) \quad(f \in Q(X)) .
\end{aligned}
$$

Il convient de rappeler que, dans la définition de $d_{0}$ et de $d_{0}^{\prime}$, on a à tenir compte des conventions faites au Chapitre $I, n^{\circ} 2$, sur les mesures de Haar à choisir dans $X$ et $X^{*}$, et sur la définition de $|\alpha|$ et de $|\gamma|$; il reviendrait au même, d'ailleurs, d'abandonner ces conventions, à condition de modifier les opérateurs $d_{0}(\alpha), d_{0}^{\prime}(\gamma)$ par des facteurs réels $>0$ qui les rendent unitaires.

Dans le cas où $f^{\prime}$ est une forme quadratique additive sur $X^{*}$ (et dans ce cas seulement), nous définirons aussi, comme suit, un relèvement $\mathbf{t}^{\prime}\left(f^{\prime}\right)$ de $t^{\prime}\left(f^{\prime}\right)$ à $M p(X)$. L'hypothèse faite sur $f^{\prime}$ entraîne que $\chi \circ f^{\prime}$ est un caractère de $X^{*}$; il y a donc un élément $a$ de $X$ tel que

$$
\chi\left(f^{\prime}\left(x^{*}\right)\right)=\chi\left(\left[a, x^{*}\right]\right)
$$

quel que soit $x^{*} \in X^{*}$. Cela posé, désignons par $t_{0}^{\prime}\left(f^{\prime}\right)$ l'opérateur défini, pour tout $\Phi \in L^{2}(X)$, par

$$
\mathbf{t}_{0}^{\prime}\left(f^{\prime}\right) \Phi(x)=\Phi(x-a)
$$

on vérifie immédiatement qu'alors $t_{0}^{\prime}\left(f^{\prime}\right)=\pi_{0}\left(t_{0}^{\prime}\left(f^{\prime}\right)\right)$. On posera, dans ces conditions

$$
\mathbf{t}^{\prime}\left(f^{\prime}\right)=\left(t^{\prime}\left(f^{\prime}\right), \mathbf{t}_{0}^{\prime}\left(f^{\prime}\right)\right) \text {. }
$$

Les relations obtenues au $n^{\circ} 13$ du Chapitre $I$ entre $d_{0}, t_{0}, d_{0}^{\prime}$, et au $n^{\circ} 33$ entre $d, t, d^{\prime}$, entraînent immédiatement des relations analogues entre $d, t, d^{\prime}$, que nous nous dispensons d'écrire. Comme au $\mathrm{n}^{\circ} 13$, on définira aussi une section de $M p(X)$ au dessus de l'ensemble $\Omega(X)$ des $s \in P s(X)$ tels que $\gamma(s)$ soit inversible, en exprimant $s$ au moyen de (33) et en posant alors 


$$
\mathbf{r}(s)=\mathbf{t}\left(f_{1}\right) \mathbf{d}^{\prime}(\gamma) \mathbf{t}\left(f_{2}\right)
$$

ou, ce qui revient au même, en écrivant

$$
\mathbf{r}(s)=\left(s, \mathbf{r}_{0}(\mu(s))\right),
$$

où $\mathbf{r}_{0}$ est défini comme au $\mathrm{n}^{\circ} 13$, et $\mu$ comme au $n^{\circ} 33$. On notera qu'en raison de la structure de corps local qu'on s'est donnée sur $k, \Omega(X)$ est ici une partie ouverte de $P s(X)$ et est donc localement compact, et même que (33) détermine un isomorphisme de variétés $k$-analytiques entre $\Omega(X)$ et $Q(X) \times \operatorname{Is}\left(X^{*}, X\right) \times Q(X)$. Le complémentaire de $\Omega(X)$ dans $P s(X)$ est donné par $\operatorname{det} \gamma(s)=0$; si $k$ n'est pas de caractéristique 2 , c'est donc un sousensemble $k$-analytique fermé de codimension 1 de $P s(X)$, et alors $\Omega(X)$ engendre $P s(X)$. Si $k$ est de caractéristique $2, \Omega(X)$ est contenu dans $P s^{+}(X)$ ou dans $P s^{-}(X)$ suivant que $m$ est pair ou impair et engendre $P s^{+}(X)$ dans le premier cas et $P s(X)$ dans le second.

On a déjà fait remarquer à la fin du $n^{\circ} 33$ que $\mu$ est injectif quand $k$ n'est pas de caractéristique 2, ce qui permet alors, si l'on veut, d'identifier $P s(X)$ avec son image dans $B_{0}(X)$; pour la même raison, on pourra alors (tout au moins du point de vue ensembliste) identifier $M p(X)$ avec sa projection sur $\mathbf{B}_{0}(X)$.

35. Pour définir une topologie sur $M p(X)$, on mettra sur $P s(X)$ la topologie déjà introduite au $\mathrm{n}^{\circ} 33$ (qui en fait un groupe localement compact, et même $k$-analytique), et sur $\mathbf{B}_{0}(X)$ la topologie induite par la topologie "forte " du groupe des automorphismes de $L^{2}(X)$. Comme il est bien connu, celle-ci est définie par le système fondamental de voisinages de 1 formé par les ensembles de la forme

$$
\left\{\mathrm{s} \mid\left\|\mathrm{s} \Phi_{i}-\Phi_{i}\right\| \leqslant 1 \quad(1 \leqslant i \leqslant n)\right\},
$$

où $\Phi_{1}, \ldots, \Phi_{n}$ sont des éléments de $L^{2}(X)$ en nombre fini quelconque. Le groupe $M p(X)$ ayant été défini ci-dessus comme sous-groupe de $P s(X) \times \mathbf{B}_{0}(X)$, on le munira de la topologie induite par celle du groupe ambiant. Il est évident, d'après cette définition, que la projection $\pi$ de $M p(X)$ sur $P s(X)$ est continue, et aussi que $(s, \mathbf{s}) \rightarrow \mathrm{s}$ est une représentation continue de $M p(X)$ dans le groupe des automorphismes de $L^{2}(X)$ muni de la topologie forte; par définition de celle-ci, cela revient à dire que $(\mathbf{S}, \Phi) \rightarrow \mathbf{S} \Phi$ est une application continue de $M p(X) \times L^{2}(X)$ dans $L^{2}(X)$.

On vérifie immédiatement que $\alpha \rightarrow \mathrm{d}_{0}(\alpha), \gamma \rightarrow \mathbf{d}_{0}^{\prime}(\gamma), f \rightarrow \mathbf{t}_{0}(\chi \circ f)$ sont des applications continues de Aut $(X)$, Is $\left(X^{*}, X\right), Q(X)$, respectivement, dans le groupe des automorphismes de $L^{2}(X)$ muni de la topologie forte; il s'ensuit que $\mathbf{d}, \mathbf{d}^{\prime}, \mathbf{t}$ sont des homéomorphismes des mêmes ensembles sur leurs images dans $M p(X)$. On en conclut aussitôt que r est un homéomorphisme de $\Omega(X)$ sur son image dans $M p(X)$, et par suite que l'application 


$$
(s, \tau) \rightarrow \tau \mathbf{r}(s)=\left(s, \tau \mathbf{r}_{0}(\mu(s))\right)
$$

est un homéomorphisme de $\Omega(X) \times T$ sur $\pi^{-1}(\Omega(X))$. Comme ce dernier ensemble est ouvert dans $M p(X)$, cela montre que $M p(X)$ est localement compact, et aussi que $\pi$ est un homomorphisme ouvert de $M p(X)$ sur $P s(X)$.

On notera que, lorsque $X$ est la somme directe $X_{1} \oplus X_{2}$ de deux espaces $X_{1}, X_{2}$ sur $k$, les résultats $d u n^{\circ} 22$ du Chapitre $I$ permettent de définir d'une manière évidente une représentation de $M p\left(X_{1}\right) \times M p\left(X_{2}\right)$ dans $M p(X)$, et que cette représentation est continue et induit sur $M p\left(X_{1}\right)$ et $M p\left(X_{2}\right)$ des isomorphismes de ces groupes sur leurs images dans $M p(X)$, avec lesquelles on pourra done les identifier chaque fois que ce sera commode.

D'autre part, il résulte des $\mathrm{n}^{\text {os }} 11-12 \mathrm{du}$ Chapitre I que, pour tout $S \in M p(X)$, l'automorphisme $\Phi \rightarrow \mathbf{S} \Phi$ de $L^{2}(X)$ induit sur $S(X)$ un automorphisme de $S(X)$; par suite, $(\mathbf{S}, \Phi) \rightarrow \mathbf{S} \Phi$ détermine une application de $M p(X) \times S(X)$ dans $S(X)$; nous allons voir maintenant que celle-ci est continue. Pour cela, il suffira de montrer que $(s, \Phi) \rightarrow \mathbf{r}(s) \Phi$ est une application continue de $\Omega(X) \times \boldsymbol{S}(X)$ dans $\boldsymbol{S}(X)$; comme d'ailleurs (33) définit un homéomorphisme entre $\Omega(X)$ et $Q(X) \times \operatorname{Is}\left(X^{*}, X\right) \times Q(X)$, et que, si $\gamma_{0}$ est arbitrairement choisi dans $\operatorname{Is}\left(X^{*}, X\right), \alpha \rightarrow \gamma_{0} \alpha$ est un homéomorphisme de $\operatorname{Aut}(X) \operatorname{sur} \operatorname{Is}\left(X^{*}, X\right)$, on est ramené à démontrer la continuité de l'application

$$
\left(f_{1}, \alpha, f_{2}, \Phi\right) \rightarrow \mathbf{t}_{0}\left(\chi \circ f_{1}\right) \mathbf{d}_{0}^{\prime}\left(\gamma_{0}\right) \mathbf{d}_{0}(\alpha) \mathbf{t}_{0}\left(\chi \circ f_{2}\right) \Phi
$$

de $Q(X) \times \operatorname{Aut}(X) \times Q(X) \times S(X)$ dans $S(X)$, ou, ce qui revient au même, celle des applications

$$
(f, \Phi) \rightarrow \mathrm{t}_{0}(\chi \circ f) \Phi, \quad(\alpha, \Phi) \rightarrow \mathrm{d}_{0}(\alpha) \Phi
$$

de $Q(X) \times S(X)$ et de $\operatorname{Aut}(X) \times S(X)$ dans $S(X)$. Quant à celle-ci, la vérification en est immédiate.

36. Dans le cas où $k$ est un corps à valuation discrète, on peut aussi, au lieu du relèvement $r_{0}$, se servir du relèvement $\mathbf{r}_{\Gamma}$ qui a été défini au $n^{\circ} 19$ du Chapitre $I$; on choisira ici pour $\Gamma$ un réseau $L$ de $X$. Dans les notations du Chapitre $I$, nous remplaçons done $G, G^{*}$, $\Gamma, \Gamma_{*} \operatorname{par} X, X^{*}, L, L_{*}$, où $L$ est un réseau dans $X$ et $L_{*}$ le réseau qui correspond à $L$ par dualité dans $X^{*}$ (c'est-à-dire l'ensemble des $x^{*} \in X^{*}$ tels que $\chi\left(\left[x, x^{*}\right]\right)=1$ quel que soit $x \in L)$. De même que nous avons substitué la considération de $P s(X)$ à celle de $B_{0}(X)$, nous substituerons ici à la considération du groupe $B_{0}(X, L)$ qu'on obtiendrait en appliquant les définitions du $\mathrm{n}^{\circ} 19$ du Chapitre I celle du sous-groupe $P s(X, L)$ de $P s(X)$ formé des éléments $s=(\sigma, f)$ de $P s(X)$ tels que $\chi \circ f$ prenne la valeur constante 1 sur $L \times L_{*}$ et que $\sigma$ induise sur $L \times L_{*}$ un automorphisme de ce groupe. On vérifie immédiatement que c'est 
là un sous-groupe ouvert $\operatorname{de} P s(X)$, compact chaque fois que $k$ n'est pas de caractéristique 2, et que l'homomorphisme $\mu$ de $P s(X)$ dans $B_{0}(X)$ applique $P s(X, L)$ dans $B_{0}(X, L)$.

Avec les notations ci-dessus, l'espace $H(G, \Gamma)$ du Chapitre $I, \mathrm{n}^{\circ} 18$, devient un espace de Hilbert $H(X, L)$, et la formule (25) du $\mathrm{n}^{\circ} 19$ de ce chapitre définit une représentation $\mathbf{r}_{L}$ de $B_{0}(X, L)$ dans le groupe des automorphismes de $H(X, L)$, donc une représentation $\mathbf{r}_{L} \circ \mu$ de $P s(X, L)$ dans ce groupe; par transport de structure au moyen de l'isomorphisme $Z^{-1}$ de $H(X, L)$ sur $L^{2}(X)$ qui est défini au $\mathrm{n}^{\circ} 18 \mathrm{du}$ même chapitre, on en tire des représentations de $B_{0}(X, L)$ et de $P s(X, L)$, notées aussi $\mathbf{r}_{L}$ et $\mathbf{r}_{L} \circ \mu$ par abus de notation, dans le groupe des automorphismes de $L^{2}(X)$. De plus, on déduit immédiatement de (25) que la représentation $\mathrm{r}_{L} \circ \mu$ de $P s(X, L)$ dans le groupe des automorphismes de $H(X, L)$, ou, ce qui revient au même, de $L^{2}(X)$, est continue quand ce dernier groupe est muni de la topologie forte. Désignons alors par $\mathbf{r}_{L}^{\prime}$ la représentation

$$
s \rightarrow\left(s, \mathbf{r}_{L}(\mu(s))\right)
$$

de $P s(X, L)$ dans $M p(X)$; il résulte de ce qui précède que $\mathbf{r}_{L}^{\prime}$ est un isomorphisme de $P s(X, L)$ sur son image dans $M p(X)$, puis que $(s, \tau) \rightarrow \tau \mathbf{r}_{L}^{\prime}(s)$ est un isomorphisme de $P s(X, L) \times T$ sur un sous-groupe ouvert de $M p(X)$. De plus, il résulte immédiatement de la fin du $n^{\circ} 21$ du Chapitre I que, pour tout $\Phi \in \mathcal{S}(X)$, l'application $s \rightarrow \mathbf{r}_{L}^{\prime}(s) \Phi$ de $P s(X, L)$ dans $S(X)$ est localement constante.

37. Nous allons maintenant étendre les résultats précédents au cas adélique. Nous reprenons naturellement ici les hypothèses et les notations des $n^{\text {os }} 29$ et 30 du Chapitre II, que nous étendons d'une manière évidente à tous les groupes algébriques définis sur le corps de base $k$; en particulier, on écrira $P s(X)_{k}, P s(X)_{v}$ pour les groupes formés par les éléments du groupe algébrique $P s(X)$ qui sont rationnels respectivement sur $k$ et sur $k_{v}$, et on écrira $P s(X)_{A}$ pour le groupe adélique attaché à $P s(X)$ de la manière habituelle. Comme au $\mathrm{n}^{\circ} 29$, on désigne par $X^{\circ}$ une base de $X$, et $\operatorname{par}\left(X^{*}\right)^{\circ}$ une base de $X^{*}$, dont on pourra supposer, pour fixer les idées, que c'est la base duale de $X^{\circ}$. Pour toute complétion $k_{v}$ de $k$ à valuation discrète, on désignera par $P s(X)_{v}^{\circ}$ le groupe formé des éléments $(\sigma, f)$ de $P s(X)_{v}$ tel que $\sigma$ induise sur $X_{v}^{\circ} \times\left(X^{*}\right)_{v}^{\circ}$ un automorphisme de ce réseau, et que $f$ induise sur ce même réseau une fonction à valeurs entières (c'est-à-dire appartenant à l'anneau $\mathbf{0}_{v}$ des entiers de $k_{v}$ ). Alors $P s(X)_{A}$ est la réunion, et même la limite inductive, des groupes

$$
P s(X)_{S}^{\circ}=\prod_{v \in S} P s(X)_{v} \times \prod_{v \notin S} P s(X)_{v}^{\circ}
$$

lorsqu'on prend pour $S$, comme d'habitude, tous les ensembles finis de complétions de $k$ contenant l'ensemble $S_{\infty}$ de celles qui sont isomorphes à $\mathbf{R}$ ou à $\mathbf{C}$. 
De même que dans le cas local (cf. $\mathrm{n}^{\circ} 33$ ), on a un homomorphisme $(w, t) \rightarrow(w, \chi(t))$ de $\mathfrak{U}(X)_{A}$ dans le groupe $A\left(X_{A}\right)$ attaché au groupe localement compact $X_{A}$ au sens du Chapitre $I, n^{\circ} 4$. De même la formule

$$
\mu_{A}((\sigma, f))=(\sigma, \chi \circ f)
$$

définit un homomorphisme $\mu_{A}$ de $P s(X)_{A}$ dans le groupe $B_{0}\left(X_{A}\right)$ attaché à $X_{A}$ au sens du Chapitre I, $\mathrm{n}^{\circ} 5$; comme dans le cas local, $\mu_{A}$ est injectif lorsque $k$ n'est pas de caractéristique 2.

Comme dans le cas local, on définira alors le groupe métaplectique $M p(X)_{A}$ comme étant le sous-groupe de $P s(X)_{A} \times \mathbf{B}_{0}\left(X_{A}\right)$ formé des éléments $(s, \mathbf{s})$ de ce produit tels que $\mu_{A}(s)=\pi_{0}(s)$; on lui donnera la topologie induite par celle du groupe ambiant lorsqu'on donne à $\mathbf{B}_{0}\left(X_{A}\right)$ la topologie forte et à $P s(X)_{A}$ la topologie adélique usuelle. On désignera de nouveau par $\pi$ la projection de ce groupe sur $P s(X)_{A}$; elle est surjective et a pour noyau le groupe $\{e\} \times \mathbf{T}$, qu'on notera aussi, plus simplement, $\mathbf{T}$.

38. On va maintenant définir un relèvement continu dans $M p(X)_{A}$ d'une partie ouverte de $P s(X)_{A}$, ce qui, de même que dans le cas local, permettra aussitôt de conclure que $M p(X)_{A}$ est localement compact et même localement homéomorphe à $P s(X)_{A} \times T$, et que $\pi$ est une application ouverte de $M p(X)_{A}$ sur $P s(X)_{A}$. Pour cela, posons, pour tout $v, \Omega_{v}=\Omega(X)_{v}$; c'est une partie ouverte non vide de $P s(X)_{v}$. Posons alors, pour tout ensemble fini $S$ de complétions de $k$, contenant $S_{\infty}$ :

$$
\Omega_{S}=\prod_{v \in S} \Omega_{v} \times \prod_{v \in S} P s(X)_{v}^{\circ}
$$

c'est une partie ouverte de $P s(X)_{S}^{\circ}$, donc de $P s(X)_{A}$. Sur chaque $\Omega_{v}$, nous disposons, d'après le $\mathrm{n}^{\circ} 34$, d'un relèvement de $\Omega_{v}$ dans $M p(X)_{v}$, que nous noterons $\mathbf{r}_{v}$; d'autre part, d'après le $\mathrm{n}^{\circ} 36$, nous disposons, pour tout $v$ tel que $k_{v}$ soit à valuation discrète, et pour tout réseau $L$ dans $X_{v}$, d'un relèvement $\mathbf{r}_{L}^{\prime}$ de $P s\left(X_{v}, L\right)$ dans $M p\left(X_{v}\right)$; de plus, d'après le $\mathrm{n}^{0} 21$ du Chapitre I, celui-ci applique $P_{s}\left(X_{v}, L\right)$ sur un sous-groupe de $M p\left(X_{v}\right)$ qui laisse invariante la fonction caractéristique du réseau $L$. Pour presque tout $v, \operatorname{Ps}(X)_{v}^{\circ}$ est un sous-groupe de $P s\left(X_{v}, L\right)$ lorsqu'on prend $L=X_{v}^{o}$; on désignera par $S_{0}$ l'ensemble (fini, et contenant $S_{\infty}$ ) des complétions de $k$ pour lesquelles il n’en est pas ainsi, et, pour $v \notin S_{0}$, on désignera par $\mathbf{r}_{v}^{\prime}$ le relèvement induit $\operatorname{sur} P s(X)_{v}^{\circ}$ par $\mathbf{r}_{L}^{\prime}$ lorsqu'on prend $L=X_{v}^{\circ}$.

D'autre part, pour tout $v$, soit $\Phi_{v}$ une fonction appartenant à $L^{2}\left(X_{v}\right)$, et supposons que, pour presque tout $v, \Phi_{v}$ soit la fonction caractéristique de $X_{v}^{\circ}$; soit $\Phi$ la fonction sur $X_{A}$, définie pour $x=\left(x_{v}\right) \in X_{A}$ par la formule

$$
\Phi(x)=\prod_{v} \Phi_{v}\left(x_{v}\right) .
$$


La mesure sur $X_{A}$ étant définie conformément aux conventions rappelées au $\mathrm{n}^{\circ}, 30 \mathrm{du}$ Chapitre II, $\Phi$ appartient à $L^{2}\left(X_{A}\right)$, et les combinaisons linéaires de fonctions de cette forme sont partout denses dans $L^{2}\left(X_{A}\right)$. Pour tout $S \supset S_{0}$, et tout $s=\left(s_{v}\right) \in \Omega_{S}$, on posera alors

$$
\mathbf{r}_{S}(s) \Phi(x)=\prod_{v \in S} \mathbf{r}_{v}\left(s_{v}\right) \Phi_{v}\left(x_{v}\right) \times \prod_{v \notin S} \mathbf{r}_{v}^{\prime}\left(s_{v}\right) \Phi_{v}\left(x_{v}\right)
$$

D'après ce qu'on a rappelé plus haut, presque tous les facteurs du second produit sont respectivement égaux aux fonctions caractéristiques des réseaux $X_{v}^{\circ}$, de sorte que la fonction ainsi définie est de la même forme que $\Phi$. Il résulte alors $d u n^{\circ} 22$ du Chapitre I que l'application $\Phi \rightarrow \mathrm{r}_{S}(s) \Phi$ définie ci-dessus pour les fonctions de la forme (36) se prolonge à un automorphisme de $L^{2}\left(X_{A}\right)$, et qu'on a défini ainsi un relèvement continu $\mathbf{r}_{S}$ de $\Omega_{S}$ dans $M p(X)_{A}$. De là on tire aussitôt les conséquences annoncées plus haut.

39. Comme dans le cas local, on va montrer maintenant que $(\mathbf{S}, \Phi) \rightarrow \mathbf{S} \Phi$ détermine une application continue de $M p\left(X_{A}\right) \times S\left(X_{A}\right)$ dans $S\left(X_{A}\right)$; de même qu'au $n^{\circ} 35$, il suffit pour cela de montrer que $(s, \Phi) \rightarrow \mathbf{r}_{S}(s) \Phi$ est une application continue de $\Omega_{S} \times \mathcal{S}\left(X_{A}\right)$ dans $\mathcal{S}\left(X_{A}\right)$.

D'après les définitions du $n^{\circ} 11 \mathrm{du}$ Chapitre I, et les remarques du $n^{\circ} 29$ du Chapitre II, $\mathcal{S}\left(X_{A}\right)$ se compose des combinaisons linéaires finies, à coefficients constants, des fonctions de la forme

$$
\Phi_{\infty}\left(x_{\infty}\right) \prod_{v \in S_{\infty}} \Phi_{v}\left(x_{v}\right)
$$

où $\Phi_{\infty}$ appartient à l'espace $\mathcal{S}\left(X_{\infty}\right)$ du produit $X_{\infty}=\prod X_{v}$ étendu aux $v \in S_{\infty}$ (de sorte que $X_{\infty}$ est un espace vectoriel de dimension finie sur $\left.\mathbf{R}\right)$, où $\Phi_{v}$ appartient à $S\left(X_{v}\right)$ pour tout $v$, et où $\Phi_{v}$ est égale, pour presque tout $v$, à la fonction caractéristique de $X_{v}^{\circ}$.

Soit alors $\Phi$ la fonction définie par (37), et soit $s \in \Omega_{S}$. De même que plus haut, $\mathbf{r}_{v}^{\prime}$ laisse $\Phi_{v}$ invariante pour presque tout $v$. Pour tout $v \in S-S_{\infty}, s_{v} \rightarrow \mathbf{r}_{v}\left(s_{v}\right) \Phi_{v}$ est une application continue, et même localement constante, de $P s(X)_{v}^{\circ}$ dans $S\left(X_{v}\right)$. Enfin, il résulte du $n^{\circ} 22$ du Chapitre I, et du $\mathrm{n}^{\circ} 35$, appliqués au produit $X_{\infty}$, que les $s_{v}$ (resp. les $\left.\mathbf{r}_{v}\left(s_{v}\right)\right)$ pour $v \in S_{\infty}$ déterminent un élément $s_{\infty}$ de $P s\left(X_{\infty}\right)$ (resp. un élément $\mathbf{r}_{\infty}\left(s_{\infty}\right)$ de $M p\left(X_{\infty}\right)$ ), leur " produit tensoriel ", de telle sorte que $s_{\infty} \rightarrow \mathbf{r}_{\infty}\left(s_{\infty}\right)$ soit une application continue; il s'ensuit, d'après le $n^{\circ} 35$, que $s_{\infty} \rightarrow \mathbf{r}_{\infty}\left(s_{\infty}\right) \Phi_{\infty}$ est une application continue dans $S\left(X_{\infty}\right)$ du produit $\prod \Omega_{v}$ étendu aux $v \in S_{\infty}$. De la combinaison de ces résultats, on conclut que $s \rightarrow \mathbf{r}_{S}(s) \Phi$ est une application continue de $\Omega_{S}$ dans $\mathcal{S}\left(X_{A}\right)$, puis que $\mathbf{S} \rightarrow \mathbf{S} \Phi$ est une application continue de $M p(X)_{A}$ dans $\mathcal{S}\left(X_{A}\right)$, quelle que soit $\Phi \in \mathcal{S}\left(X_{A}\right)$.

Pour achever de démontrer la continuité de $(s, \Phi) \rightarrow \mathbf{r}_{\mathrm{S}}(s) \Phi$, il suffit alors de vérifier ce qui suit : soient $K$ une partie compacte de $\Omega_{S}$, et $U$ un voisinage convexe de 0 dans $\boldsymbol{S}\left(X_{A}\right)$; alors l'ensemble $U^{\prime}$ des $\Phi \in \mathcal{S}\left(X_{A}\right)$ tels que $\mathbf{r}_{S}(s) \Phi \in U$ quel que soit $s \in K$ est un voisinage de 
0 dans $S\left(X_{A}\right)$. Comme $U^{\prime}$ est convexe, cela revient, d'après la définition de la topologie de $S\left(X_{A}\right)$ comme limite inductive de celles des $S\left(H, H^{\prime}\right)$ (cf. $\mathrm{n}^{\circ} 11$ ), à montrer que $U^{\prime} \cap S\left(H, H^{\prime}\right)$ est un voisinage de 0 dans $S\left(H, H^{\prime}\right)$ quels que soient $H$ et $H^{\prime}$. Or, pour $K, H$ et $H^{\prime}$ donnés, il y a un ensemble fini $S^{\prime}$ de complétions de $k$ ayant les propriétés suivantes : $(a)$ pour tout $s=\left(s_{v}\right) \in K$ et tout $v \notin S^{\prime}$, on a $s_{v} \in P s(X)_{v}^{\circ} ;(b)$ toute fonction appartenant à $S\left(H, H^{\prime}\right)$ est combinaison linéaire de fonctions de la forme (37) où $\Phi_{v}$ est la fonction caractéristique de $X_{v}^{\circ}$ pour tout $v \notin S^{\prime}$. Dans ces conditions, l'assertion qu'il s'agit de démontrer est une conséquence immédiate des propriétés de continuité démontrées au $n^{\circ} 35$ pour le cas local.

40. D'autre part, on peut appliquer les résultats du Chapitre $I, \mathrm{n}^{0 \mathrm{~s}} 16-19$, ̀̀ $G=X_{A}$, $G^{*}=X_{A}^{*}, \Gamma=X_{k}, \Gamma_{*}=X_{k}^{*}$; il est immédiat qu'alors l'homomorphisme $\mu_{A}$ de $P s(X)_{A}$ dans $B_{0}\left(X_{A}\right)$, défini au $\mathrm{n}^{\circ} 37$, applique $P s(X)_{k}$ dans le sous-groupe de $B_{0}\left(X_{A}\right)$ qui, avec les notations du $n^{\circ} 19$, s'écrit $B_{0}\left(X_{A}, X_{k}\right)$. De même que dans les situations analogues étudiées plus haut, on peut donc, au moyen du relèvement $\mathbf{r}_{\Gamma}$ de $B_{0}(G, \Gamma)$ défini au $\mathrm{n}^{\circ} 19$, définir ici un relèvement de $P s(X)_{k}$ dans $\mathbf{B}_{0}(G)$, et par suite un relèvement de $P s(X)_{k}$ dans $M p(X)_{A}$, qu'on notera $\mathbf{r}_{k}$. Nous allons maintenant expliciter celui-ci; il suffira d'ailleurs pour cela d'exprimer $\mathbf{r}_{k}(s) \Phi$ au moyen de $\Phi$ pour $\Phi \in S\left(X_{A}\right), s \in P s(X)_{k}$. Conformément aux définitions du Chapitre I, nous devons donc introduire la fonction

$$
\Theta\left(x, x^{*}\right)=\sum_{\xi \in X_{k}} \Phi(x+\xi) \chi\left(\left[\xi, x^{*}\right]\right) \quad\left(x \in X_{A}, x^{*} \in X_{A}^{*}\right)
$$

ici, comme on l'a déjà noté dans le cas plus général étudié au Chapitre $I, n^{\circ} 18$, la série du second membre est uniformément convergente sur tout compact en vertu de l'hypothèse $\Phi \in S\left(X_{A}\right)$. On a alors la formule (cas particulier de (23) $\mathrm{du} \mathrm{n}^{\circ} 18$ ) :

$$
\Phi(x)=\int_{X_{A}^{*} / X_{k}^{*}} \Theta\left(x, x^{*}\right) d \dot{x}^{*}
$$

où $d \dot{x}^{*}$ est la mesure sur le groupe compact $X_{A}^{*} / X_{k}^{*}$ qui donne la valeur 1 à la mesure du groupe. Soit $s=(\sigma, f)$ un élément de $P s(X)_{k}$, et écrivons comme d'habitude $\sigma=\left(\begin{array}{l}\alpha \beta \\ \gamma \delta\end{array}\right)$. D'après le $\mathbf{n}^{\circ} 19$, la fonction $\Phi^{\prime}=\mathbf{r}_{k}(s) \Phi$ est définie par les formules

ou encore

$$
\begin{aligned}
& \Theta^{\prime}(z)=\Theta(z \sigma) \chi(f(z)) \quad\left(z=\left(x, x^{*}\right) \in X_{A} \times X_{A}^{\prime}\right), \\
& \Phi^{\prime}(x)=\int \Theta^{\prime}\left(x, x^{*}\right) d \dot{x}^{*}, \\
& \Phi^{\prime}(x)=\int \Theta\left(x \alpha+x^{*} \gamma, x \beta+x^{*} \delta\right) \chi\left(f\left(x, x^{*}\right)\right) d \dot{x}^{*},
\end{aligned}
$$


l'intégrale étant prise sur $X_{A}^{*} / X_{k}^{*}$. Soit $N$ le noyau de $\gamma$ dans $X^{*}$; soit $Y=X^{*} / N$. On peut, d'une manière évidente, identifier $N_{A} / N_{k}$ avec un sous-groupe fermé de $X_{A}^{*} / X_{k}^{*}$ et $Y_{A} / Y_{k}$ avec le quotient de $X_{A}^{*} / X_{k}^{*}$ par $N_{A} / N_{k}$; en désignant par $\bar{x}^{*}$ l'image de $\dot{x}^{*}$ dans ce dernier quotient, on pourra done écrire

$$
\Phi^{\prime}(x)=\int \Psi\left(x, \bar{x}^{*}\right) d \bar{x}^{*}
$$

où $\Psi$ est donnée par la formule

$$
\Psi\left(x, \bar{x}^{*}\right)=\int_{N_{A} / N_{k}} \sum_{\xi \in X_{k}} \Phi\left(x \alpha+x^{*} \gamma+\xi\right) \chi\left(\left[\xi, x \beta+\left(x^{*}+n\right) \delta\right]+f\left(x, x^{*}+n\right)\right) d \dot{n}
$$

ici $\dot{n}$ désigne l'image dans $N_{A} / N_{k}$ de $n \in N_{A}$, et $d \dot{n}$ est la mesure dans $N_{A} / N_{k}$ pour laquelle $N_{A} / N_{k}$ a la mesure 1; de même, $d \bar{x}^{*}$ est la mesure dans $Y_{A} / Y_{k}$ pour laquelle $Y_{A} / Y_{k}$ a la mesure 1 .

On a d'ailleurs, en vertu de (32) :

$$
f\left(x, x_{1}^{*}+x_{2}^{*}\right)=f\left(0, x_{1}^{*}\right)+f\left(x, x_{2}^{*}\right)+\left[x_{1}^{*} \gamma, x \beta+x_{2}^{*} \delta\right],
$$

d'où, pour $n \in N$ :

$$
f\left(x, x^{*}+n\right)=f(0, n)+f\left(x, x^{*}\right) .
$$

En particulier, il s'ensuit que $f(0, n)$ est une forme additive sur $N$. Comme $f$ est rationnelle sur $k$, on peut donc définir un caractère $\varphi$ de $N_{A} / N_{k}$ en posant $\varphi(\dot{n})=\chi(f(0, n))$ pour $n \in N_{A}$, puis prolonger $\varphi$ à un caractère de $X_{A}^{*} / X_{k}^{*}$. Il revient au même de dire qu'il y a un caractère de $X_{A}^{*}$, prenant la valeur 1 sur $X_{k}^{*}$, qui coincide avec $\chi(f(0, n))$ sur $N_{A}$, ou encore qu'il existe $\xi_{0} \in X_{k}$ tel que l'on ait

$$
\chi(f(0, n))=\chi\left(\left[\xi_{0}, n\right]\right)
$$

quel que soit $n \in N_{A}$. Bien entendu, si $k$ n'est pas de caractéristique 2, la forme additive $f(0, n)$ sur $N$ se réduit à 0 , de sorte qu'on peut prendre $\xi_{0}=0$.

Cela posé, l'expression ci-dessus pour $\Psi$ s'écrit aussi

$$
\Psi\left(x, \bar{x}^{*}\right)=\int \sum_{\xi} \Phi\left(x \alpha+x^{*} \gamma+\xi\right) \chi\left(\left[\xi, x \beta+x^{*} \delta\right]+f\left(x, x^{*}\right)\right) \chi\left(\left[\xi \delta^{*}+\xi_{0}, n\right]\right) d \dot{n}
$$

ou plus simplement

$$
\Psi\left(x, \bar{x}^{*}\right)=\sum_{\xi \in L} \Phi\left(x \alpha+x^{*} \gamma+\xi\right) \chi\left(\left[\xi, x \beta+x^{*} \delta\right]+f\left(x, x^{*}\right)\right),
$$

où la sommation est étendue à l'ensemble $L \operatorname{des} \xi \in X_{k}$ tels que l'on ait 13-642946 Acta mathematica. 111. Imprimé le 3 juin 1964. 


$$
\left[\xi \delta^{*}+\xi_{0}, n\right]=0
$$

pour tout $n \in N$, ou encore tels que $\xi \delta^{*}+\xi_{0}$ appartienne au sous-espace $N_{*}$ de $X$ orthogonal au noyau $N$ de $\gamma$ dans $X^{*} ; N_{*}$ n'est pas autre chose que l'image $X^{*} \gamma^{*}$ de $X^{*}$ par $\gamma^{*}$. On peut dire aussi que $L$ est l'ensemble des $\xi \in X_{k}$ tels que l'équation

$$
\xi \delta^{*}+\xi_{0}=\xi^{*} \gamma^{*}
$$

ait une solution $\xi^{*}$ dans $X_{k}^{*}$; comme on a

$$
\sigma^{-1}=\left(\begin{array}{rr}
\delta^{*} & -\beta^{*} \\
-\gamma^{*} & \alpha^{*}
\end{array}\right)
$$

cela revient à dire que $\left(\xi, \xi^{*}\right) \sigma^{-1}$ est de la forme $\left(-\xi_{0}, \xi_{1}^{*}\right)$ avec $\xi_{1}^{*} \in X_{k}$. En définitive, $L$ est donc l'image de $X_{k}^{*}$ par l'application

$$
\xi_{1}^{*} \rightarrow-\xi_{0} \alpha+\xi_{1}^{*} \gamma
$$

de $X_{k}^{*}$ dans $X_{k}$. En tenant compte de (32), on peut alors écrire

$$
\Psi\left(x, \bar{x}^{*}\right)=\sum \Phi\left(\left(x-\xi_{0}\right) \alpha+\left(x^{*}+\xi_{1}^{*}\right) \gamma\right) \chi\left(f\left(x-\xi_{0}, x^{*}+\xi_{1}^{*}\right)-\left[\xi_{0}, x^{*}\right]\right)
$$

où la sommation est étendue à des valeurs de $\xi_{1}^{*}$ prises dans un système complet de représentants de $X_{k}^{*}$ modulo $N_{k}$. Observons maintenant qu'en vertu de la définition de $\xi_{0}$, la fonction

$$
\chi\left(f\left(x, x^{*}\right)-\left[\xi_{0}, x^{*}\right]\right)
$$

ne change pas si on y remplace $x^{*}$ par $x^{*}+n$, avec $n \in N_{A}$. On peut donc définir une fonction $\Omega \operatorname{sur} X_{A} \times Y_{A}$ en posant

$$
\Omega(x, y)=\Phi\left(x \alpha+x^{*} \gamma\right) \chi\left(f\left(x, x^{*}\right)-\left[\xi_{0}, x^{*}\right]\right)
$$

chaque fois que $x \in X_{A}, x^{*} \in X_{A}^{*}$, et que $y$ est l'image de $x^{*}$ dans $Y_{A}=X_{A}^{*} / N_{A}$. On a alors, avec ces mêmes notations

et par suite

$$
\Psi\left(x, \bar{x}^{*}\right)=\sum_{\eta \in Y_{k}} \Omega\left(x-\xi_{0}, y+\eta\right),
$$

$$
\Phi^{\prime}(x)=\int_{Y_{A}} \Omega\left(x-\xi_{0}, y\right) d y
$$

où $d y$ est la mesure de Tamagawa sur $Y_{A}$ (celle pour laquelle $Y_{A} / Y_{k}$ est de mesure 1). Comme $x^{*} \rightarrow x^{*} \gamma$ détermine, par passage au quotient, un isomorphisme de $Y$ sur l'image $Z=X^{*} \gamma$ de $X^{*}$ par $\gamma$, on obtient en définitive 


$$
\mathbf{r}_{k}(s) \Phi(x)=\int_{z_{A}} \Phi\left(\left(x-\xi_{0}\right) \alpha+z\right) \psi\left(x-\xi_{0}, z\right) d z
$$

où $Z=X^{*} \gamma, d z$ est la mesure de Tamagawa sur $Z_{A}$, et $\psi$ est le caractère du second degré de $X_{A} \times Z_{A}$ défini par

$$
\psi\left(x, x^{*} \gamma\right)=\chi\left(f\left(x, x^{*}\right)-\left[\xi_{0}, x^{*}\right]\right) \quad\left(x \in X_{A}, x^{*} \in X_{A}^{*}\right) .
$$

41. L'application du théorème $4 \mathrm{du}$ Chapitre $\mathrm{I}, \mathrm{n}^{\circ} 19$, va nous donner maintenant le résultat que nous avions principalement en vue dans ce mémoire. Ce théorème donne d'abord :

$$
\sum_{\xi \in X_{k}} \Phi(\xi)=\sum_{\xi \in X_{k}} \mathbf{r}_{k}(s) \Phi(\xi)
$$

égalité qui est valable chaque fois que $s \in P s(X)_{k}$ et $\Phi \in S\left(X_{A}\right)$; elle se réduit à la formule de Poisson lorsque $s=d^{\prime}(\gamma), \gamma$ étant un isomorphisme de $X_{k}^{*}$ sur $X_{k}$. De là, ou, ce qui revient du même, du corollaire du théorème 4 , nous voulons maintenant déduire ce qui suit :

THÉoRìme 6. Soient $X_{k}$ un espace vectoriel de dimension finie sur $k$, et $\Phi$ une fonction appartenant à $\mathbf{S}\left(X_{A}\right)$. Soit $\Theta$ la fonction sur $M p(X)_{A}$, définie, pour tout $\mathbf{S} \in M p(X)_{A}$, par la formule

$$
\Theta(\mathbf{S})=\sum_{\xi \in X_{k}} \mathbf{S} \Phi(\xi)
$$

Alors $\Theta$ est une fonction continue sur $M p(X)_{A}$, invariante par les translations à gauche déterminées par les éléments de $M p(X)_{A}$ de la forme $\mathbf{r}_{k}(s)$, avec $s \in P s(X)_{k}$.

L'invariance de $\Theta$ est évidente d'après (39), ou d'après le corollaire du théorème 4 du $n^{\circ} 19$; il n'y a à démontrer que sa continuité, que nous déduirons des lemmes suivants.

Lемм 4. Soit $\left(a_{n}\right)_{n \in \mathbf{N}}$ une suite de nombres réels $a_{n}>0$. Alors il existe une fonction $\varphi \in \boldsymbol{S}(\mathbf{R})$ telle que l'on ait, quel que soit $x$ :

$$
\varphi(x) \geqslant \inf _{n \in \mathbf{N}}\left(a_{n}|x|^{-n}\right) .
$$

En effet, soit $f(x)=\inf \left(a_{n}|x|^{-n}\right)$; soit $g$ une fonction indéfiniment différentiable sur $\mathbf{R}$, ̀̀ valeurs $\geqslant 0$, de support contenu dans $[-1,+1]$, et telle que $\int g d x=1$, et soit $h=f * g$. On aura

$$
\begin{array}{lll}
f(x-1) \geqslant h(x) \geqslant f(x+1) & \text { pour } & x \geqslant+1, \\
f(x-1) \leqslant h(x) \leqslant f(x+1) & \text { pour } \quad x \leqslant-1 .
\end{array}
$$

De plus, si $D=d / d x$, on a $D^{p} h=f * D^{p} g$ quel que soit $p \geqslant 0$, d'où on conclut immédiatement que $\left|x^{n} D^{p} h\right|$ est borné pour tout $n \geqslant 0$ et tout $p \geqslant 0$, donc $h \in \boldsymbol{S}(\mathbf{R})$. Soit $h_{0} \in \boldsymbol{S}(\mathbf{R})$ tel que $h_{0}(x) \geqslant 0$ pour tout $x$ et $h_{0}(x) \geqslant a_{0}$ pour $-2 \leqslant x \leqslant+2$. On aura, quel que soit $x$ : 


$$
f(x) \leqslant h(x-1)+h(x+1)+h_{0}(x)
$$

ce qui démontre le lemme $\left.{ }^{1}\right)$.

LEMME 5. Soient $G$ un groupe abélien localement compact et $C$ une partie compacte de $\mathfrak{S}(G)$. Alors il existe $\Phi_{0} \in \mathfrak{S}(G)$ telle que $|\Phi(x)| \leqslant \Phi_{0}(x)$ quels que soient $\Phi \in C$ et $x \in G$.

Avec les notations qui ont servi à définir $S(G)$ au $n^{\circ} 11$, on sait (cf. Bruhat [1]) que toute partie compacte de $S(G)$ est contenue dans un espace $S\left(H, H^{\prime}\right)$; il suffit donc de démontrer le lemme pour un groupe élémentaire $G=\mathbf{R}^{n} \times \mathbf{Z}^{p} \times T^{q} \times F$, où $F$ est un groupe fini. Soit $x \in G$; soient $x_{1}, \ldots, x_{n}, y_{1}, \ldots, y_{\mathfrak{y}}$ ses coordonnées relatives aux facteurs $\mathbf{R}, \mathbf{Z}$ de $G$; posons

et, pour tout $n \in \mathbf{N}$ :

$$
r(x)=\sum_{i=1}^{n} x_{i}^{2}+\sum_{j=1}^{p} y_{j}^{2}
$$

$$
a_{n}=\sup _{x \in G, \Phi \in C}\left|r(x)^{n} \Phi(x)\right|
$$

d'après la définition de $S(G)$, on a $a_{n}<+\infty$ pour tout $n$. Pour cette suite $\left(a_{n}\right)$, soit $\varphi$ une fonction de $S(R)$ ayant la propriété énoncée dans le lemme 4. Alors $\Phi_{0}=\varphi \circ r$ a la propriété voulue.

Revenons à la démonstration du théorème 6. Il résulte immédiatement des définitions que, pour tout $x_{0} \in X_{A}, \Phi \rightarrow \Phi\left(x_{0}\right)$ est une application continue de $S\left(X_{A}\right)$ dans $\mathbf{C}$. Comme d'autre part on a montré que, pour chaque $\Phi \in S\left(X_{A}\right), \mathbf{S} \rightarrow \mathbf{S} \Phi$ est une application continue de $M p(X)_{A}$ dans $S\left(X_{A}\right)$, il s'ensuit que chacun des termes de la série qui définit $\Theta$ est une fonction continue sur $M p(X)_{A}$. Soit $C$ une partie compacte de $M p(X)_{A}$; si $\Phi \in S\left(X_{A}\right)$, l'image de $C$ par $\mathbf{S} \rightarrow \mathbf{S} \Phi$ est une partie compacte de $S\left(X_{A}\right)$, et il résulte donc du lemme 5 qu'il existe $\Phi_{0} \in S\left(X_{A}\right)$ telle que $|S \Phi| \leqslant \Phi_{0}$ quel que soit $S \in C$. La série qui définit $F(S)$ est donc, pour $S \in C$, majorée terme à terme par la série $\sum \Phi_{0}(\xi)$. Celle-ci étant convergente d'après ce qui précède, cela achève la démonstration.

La définition de fonctions modulaires au moyen de séries thêta est naturellement un cas particulier du mode de définition de fonctions automorphes qui est contenu dans le théorème 6 .

\section{Réduction du groupe métaplectique}

42. Aussi bien dans le cas local que dans le cas adélique, le groupe métaplectique est une extension centrale du groupe pseudosymplectique par le groupe $T$. On va montrer maintenant qu'en général cette extension n'est pas triviale, mais que du moins elle se

(1) Cette démonstration m'a été communiquée par J. Dieudonné. 
ramène toujours à une extension par le groupe à deux éléments $\{ \pm 1\}$, ou, comme on pourrait le dire aussi, à un revêtement à deux feuillets.

Nous aurons besoin d'un lemme de théorie des groupes, sans rapport avec ce qui précède.

Lем ме 6. Soient $G$ un groupe, et $U$ une partie de $G$, tels que $U^{-1} \cap U a \cap U b \cap U c \neq \emptyset$ quels que soient $a, b, c$ dans $G$. Soit $R$ l'ensemble des éléments $\left(u, u^{\prime}, u^{\prime \prime}\right)$ de $U \times U \times U$ tels que $u^{\prime \prime}=u u^{\prime}$. Alors $G$ est engendré par les éléments de $U$ et peut être identifié avec le groupe défini par ces générateurs et par les relations $u^{\prime \prime}=u u^{\prime}$ pour $\left(u, u^{\prime}, u^{\prime \prime}\right) \in R$.

Soit $x \in G$; soit $v \in U^{-1} \cap U x^{-1}$; alors on a $x=v^{-1}(v x)$ et $v^{-1} \in U, v x \in U$, ce qui justifie la première assertion. Soit $\bar{G}$ le groupe engendré par des éléments $\bar{u}$ correspondant biunivoquement aux $u \in U$, et défini par ces générateurs et par les relations $\bar{u}^{\prime \prime}=\bar{u} \bar{u}^{\prime}$ correspondant aux éléments $\left(u, u^{\prime}, u^{\prime \prime}\right)$ de $R$; alors $\bar{u} \rightarrow u$ détermine un homomorphisme $h$ de $\bar{G}$ sur $G$, et nous avons à démontrer que le noyau $N$ de $h$ se réduit à l'élément neutre $\bar{e} \operatorname{de} \bar{G}$. Montrons d'abord que, si un élément de $N$ est de la forme $\bar{u}_{0}^{-1} \bar{u}_{1} \bar{u}_{2} \ldots \bar{u}_{n}$, c'est $\bar{e}$. Pour $n=1$ c'est trivial; pour $n=2$, cela résulte de la définition de $\bar{G}$; nous pouvons donc procéder par récurrence. Un tel élément de $N$ étant donné, soit $v$ un élément de l'ensemble

$$
U^{-1} \cap U u_{0}^{-1} \cap U u_{1}^{-1} \cap U u_{2}^{-1} u_{1}^{-1}
$$

Les éléments $u=v^{-1}, u^{\prime}=v u_{0}, u^{\prime \prime}=v u_{1}, u^{\prime \prime \prime}=v u_{1} u_{2}$ de $G$ appartiennent donc à $U$, et on a les relations

$$
u_{0}=u u^{\prime}, \quad u_{1}=u u^{\prime \prime}, \quad u^{\prime \prime} u_{2}=u^{\prime \prime \prime}, \quad u^{\prime}=u^{\prime \prime \prime} u_{3} \ldots u_{n}
$$

ce qui entraîne (en vertu de la définition de $\bar{G}$ en ce qui concerne les trois premières de ces relations, et en vertu de l'hypothèse de récurrence en ce qui concerne la dernière)

$$
\bar{u}_{0}=\bar{u} \bar{u}^{\prime}, \quad \bar{u}_{1}=\bar{u} \bar{u}^{\prime \prime}, \quad \bar{u}^{\prime \prime} \bar{u}_{2}=\bar{u}^{\prime \prime \prime}, \quad \bar{u}^{\prime}=\bar{u}^{\prime \prime \prime} \bar{u}_{3} \ldots \bar{u}_{n},
$$

et par suite $\bar{u}_{0}=\bar{u}_{1} \bar{u}_{2} \ldots \bar{u}_{n}$, comme nous l'avions annoncé. Montrons maintenant que, si un élément de $N$ est de la forme $\bar{u}_{1} \ldots \bar{u}_{n}$ (où $n \geqslant 1$ ), c'est $\bar{e}$. En effet, cette hypothèse entraîne que $\bar{u}^{-1} \bar{u} \bar{u}_{1} \ldots \bar{u}_{n}$ est dans $N$ quel que soit $u \in U$, d'où la conclusion annoncée en vertu de ce qu'on a démontré précédemment. Soit alors $V=U \cup U^{-1}$, et soit $S$ l'ensemble des éléments $\left(v, v^{\prime}, v^{\prime \prime}\right)$ de $V \times V \times V$ tels que $v^{\prime \prime}=v v^{\prime}$. Prolongeons à $V$ l'application $u \rightarrow \bar{u}$ de $U$ dans $\bar{G}$ en posant $\bar{v}=\bar{u}^{-1}$ pour $v=u^{-1}, u \in U$, ce qui, d'après ce qu'on a démontré, coïncide bien sur $U \cap U^{-1}$ avec l'application $u \rightarrow \bar{u}$ qu'il s'agissait de prolonger. D'après ce qu'on a déjà démontré, on vérifie immédiatement, par l'examen des différents cas possibles, que $\bar{v}^{\prime \prime}=\bar{v} \bar{v}^{\prime}$ chaque fois que $\left(v, v^{\prime}, v^{\prime \prime}\right) \in S$. Mais alors le raisonnement fait plus haut pour $U$ et $R$ 
s'applique à $V$ et $S$ et montre que tout élément de $N$ de la forme $\bar{v}_{1} \ldots \bar{v}_{n}$ est égal à $\bar{e}$. Comme tout élément de $\bar{G}$ peut se mettre sous cette forme, cela achève la démonstration.

Il est clair que les hypothèses du lemme 6 sont satisfaites lorsqu'on prend pour $G$ un groupe analytique sur un corps local $k$, et pour $U$ le complémentaire d'une réunion de sousvariétés analytiques de $G$ de codimension $\geqslant 1$. En particulier, il en sera ainsi, d'après les $\mathrm{n}^{\text {os }} 31-32$, quand $k$ est un corps local de caractéristique autre que $2, X$ un espace vectoriel de dimension finie sur $k$, et qu'on prend $G=P s(X), U=\Omega(X)$, ou encore quand $k$ est un corps local de caractéristique $2, X$ un espace de dimension paire sur $k$, et qu'on prend $G=P s^{\dagger}(X), U=\Omega(X)$.

43. Revenons à la question posée au début du chapitre, et soit d'abord $X$ de dimension finie sur un corps local $k$ de caractéristique autre que 2 , de sorte qu'on peut identifier $P s(X)$ avec le groupe symplectique $S p(X)$. Suivant l'usage, on dira que $M p(X)$ est une extension triviale de $S p(X)$ par $T$ si l'on peut écrire $M p(X)=S p_{1}(X) \times \mathbf{T}$, où $S p_{1}(X)$ est un sous-groupe de $M p(X)$ sur lequel $\pi$ induit un isomorphisme de $S p_{1}(X)$ sur $S p(X)$. Pour qu'il en soit ainsi au sens de la théorie des groupes (resp. au sens de la théorie des groupes topologiques), il faut et il suffit qu'on puisse définir sur $M p(X)$ un homomorphisme (resp. un homomorphisme continu) de $M p(X)$ dans $\mathbf{T}$, se réduisant à l'identité sur T; en notant $\theta$ l'isomorphisme $(e, t) \rightarrow t$ de $\mathbf{T}$ sur $T$, il revient au même de dire qu'il doit exister un caractère (resp. un caractère continu) de $M p(X)$, coïncidant avec $\theta$ sur $\mathbf{T}$.

Plus généralement, supposons qu'il existe un caractère $\varphi$ de $M p(X)$, continu ou non, qui coïncide avec $\theta^{n}$ sur $\mathbf{T}, n$ étant un entier $>0$, et soit $N$ le noyau de $\varphi$; alors on a $M p(X)=N \cdot \mathbf{T} ; N \cap \mathbf{T}$ est le sous-groupe $\mathbf{T}_{n}$ d'ordre $n$ de $\mathbf{T} ; \boldsymbol{\pi}$ induit sur $N$ un homomorphisme de $N$ sur $S p(X)$ de noyau $\mathbf{T}_{n}$; si de plus $\varphi$ est continu, $\dot{N}$ est fermé dans $M p(X)$, et $\pi$ induit sur $N$ un homomorphisme ouvert de $N$ sur $S p(X)$, de noyau $\mathbf{T}_{n}$, qui est en même temps un "isomorphisme local ", de sorte qu'on peut dire que $N$ est un " revêtement à $n$ feuillets » de $S p(X)$. Nous allons maintenant appliquer à la construction d'un tel caractère $\varphi$ le lemme $6 \mathrm{du} \mathrm{n}^{\circ} 42$, en le combinant avec le théorème 3 du Chapitre $\mathrm{I}, \mathrm{n}^{\circ} 15$, et avec la détermination de $\gamma(f)$ effectuée au Chapitre II.

Comme, d'après le lemme $6, S p(X)$ est engendré par $\Omega(X)$, le caractère $\varphi$ est entièrement déterminé par les valeurs de $\psi=\varphi \circ$ r sur $\Omega(X)$. De plus, compte tenu de la différence de notations entre les Chapitres I et III, on déduit du théorème 3 du Chapitre I, $n^{\circ} 15$, que, si $s^{\prime \prime}=s s^{\prime}$, avec $s, s^{\prime}, s^{\prime \prime}$ dans $\Omega(X)$, on a

$$
\mathbf{r}(s) \mathbf{r}\left(s^{\prime}\right)=\gamma\left(f_{0}\right) \mathbf{r}\left(s^{\prime \prime}\right),
$$

où $f_{0}$ est une certaine forme quadratique sur $X$, associée au morphisme $\gamma(s)^{-1} \gamma\left(s^{\prime \prime}\right) \gamma\left(s^{\prime}\right)^{-1}$ de $X$ sur $X^{*}$. Il s'ensuit que, si $\psi$ est comme ci-dessus, on a, dans ces conditions : 


$$
\psi\left(s^{\prime \prime}\right)=\gamma\left(f_{0}\right)^{-n} \psi(s) \psi\left(s^{\prime}\right)
$$

Réciproquement, on conclut immédiatement du lemme 6 que, si $\psi$ est une application de $\Omega(X)$ dans $T$ satisfaisant à (40), on peut, d'une manière et d'une seule, déterminer un caractère $\varphi$ de $M p(X)$ qui satisfasse à $\varphi \circ \mathbf{r}=\psi$ et qui coïncide avec $\theta^{n}$ sur $\mathbf{T}$; de plus, $\varphi$ est continu si $\psi$ est continue.

Soit d'abord $k=\mathbf{C}$; d'après le $\mathrm{n}^{\circ} 26$ du Chapitre II, on a alors $\gamma(f)=1$ pour toute forme quadratique non dégénérée $f$ sur $X$. On satisfait done à $(40)$ en prenant $n=1, \psi=1$; il y a donc un caractère et un seul de $M p(X)$ qui prenne la valeur 1 sur $\mathbf{r}(\Omega(X))$ et qui coïncide avec $\theta$ sur $\mathbf{T} ;$ on désignera ce caractère par $\varphi_{1}$, et son noyau par $S p_{1}(X)$. On a $M p(X)=$ $S p_{1}(X) \times \mathbf{T} ; S p_{1}(X)$ est un sous-groupe fermé de $M p(X)$, isomorphe à $S p(X)$, et est engendré $\operatorname{par} \mathbf{r}(\Omega(X))$.

Pour $k \neq \mathbf{C}$, supposons d'abord que -1 soit un carré dans $k$; d'après le $n^{\circ} 25$ du Chapitre II, on a alors $\gamma(f)= \pm 1$ pour toute forme quadratique non dégénérée $f$ sur $X$. On satisfait donc à (40) en prenant $n=2, \psi=1$. On notera $\varphi_{2}$ le caractère correspondant de $M p(X)$, et $S p_{2}(X)$ son noyau; c'est un sous-groupe fermé de $M p(X)$ et un " revêtement à deux feuillets 》 de $S p(X)$.

Supposons maintenant que -1 ne soit pas un carré dans $k$. Choisissons une base de $X$ sur $k$, et, pour tout $s \in \Omega(X)$, désignons par $D(s)$ le déterminant de $\gamma(s)$ par rapport à cette base de $X$ et à la base de $X^{*}$ duale de celle-ci; par définition de $\Omega(X)$, on a $D(s) \neq 0$ pour tout $s \in \Omega(X)$. La formule $(28) d u n^{\circ} 28$, jointe à ce qu'on a rappelé plus haut, montre alors qu'on satisfait à $(40)$ en prenant $n=2$ et

$$
\psi(s)=(D(s) /-1) \gamma\left(q_{1}\right)^{2 m}
$$

pour tout $s \in \Omega(X)$, $m$ désignant la dimension de $X$ et $q_{1}$ la forme $q_{1}(x)=x^{2}$ sur $k$; $\psi$ est continue, et même localement constante, sur $\Omega(X)$. Comme plus haut, on notera $\varphi_{2}$ le caractère correspondant de $M p(X)$, et $S p_{2}(X)$ son noyau; celui-ci a les mêmes propriétés que précédemment.

Passons au cas où $k$ est un corps local de caractéristique 2; dans ce cas, désignons par $M p^{+}(X)$ l'image réciproque de $P_{s^{+}}(X)$ dans $M p(X)$, qui est un sous-groupe ouvert d'indice 2 de $M p(X)$. Soit d'abord $X$ de dimension paire sur $k$; alors le lemme 6 s'applique à $P s^{+}(X)$ et $\Omega(X)$, et, d'après le $\mathbf{n}^{\circ} 25$, on a $\gamma(f)= \pm 1$ pour toute forme non dégénérée $f$ sur $X$; on peut donc, dans (40), prendre $n=2$ et $\psi=1$, ce qui définit un caractère $\varphi_{2}$ de $M p^{+}(X)$, coïncidant avec $\theta^{2}$ sur $\mathrm{T}$; son noyau $P s_{2}^{+}(X)$ est un sous-groupe fermé de $M p^{+}(X)$, et un "revêtement à deux feuillets» de $P_{S^{+}}(X)$. Si $X$ est de dimension impaire, on peut appliquer ce qui précède à l'espace $X^{\prime}=X \oplus k$, qui est de dimension paire; le caractère $\varphi_{2}^{\prime}$ de $M p^{+}\left(X^{\prime}\right)$, 
défini comme on vient de le dire, induit alors sur $M p^{+}(X)$, considéré comme sous-groupe de $M p^{+}\left(X^{\prime}\right)$ (cf. $\mathrm{n}^{\circ} 35$ ) un caractère analogue $\varphi_{2}$, dont on notera encore le noyau $P s_{2}^{+}(X)$. Enfin, si $X$ est de dimension 1 sur $k$, tout élément de $P s^{+}(X)$ est de la forme $d(\alpha) t(f)$; si on désigne en ce cas par $P s_{1}^{+}(X)$ l'ensemble des éléments de $M p(X)$ de la forme $\mathrm{d}(\alpha) \mathbf{t}(f)$, on vérifie immédiatement que c'est là un sous.groupe fermé de $M p^{+}(X)$, isomorphe à $P s^{+}(X)$, et qu'on a $M p^{+}(X)=P s_{1}^{+}(X) \times \mathbf{T}$.

44. On va montrer maintenant que les résultats du $n^{\circ} 43$ sont « les meilleurs possibles » au sens suivant : si $k$ n'est pas de caractéristique $2, M p(X)$ n'est pas une extension triviale de $S p(X)$ à moins que $k=\mathrm{C}$; si $k$ est de caractéristique $2, M p^{+}(X)$ n'est pas une extension triviale de $P s^{+}(X)$ à moins que $X$ ne soit de dimension 1 sur $k$. D'ailleurs, si $X=X_{1} \oplus X_{2}$ et qu'il existe un caractère $\varphi$ de $M p(X)$ (resp. de $M p^{+}(X)$ ) coïncidant avec $\theta$ sur $\mathbf{T}$, celui-ci induira un caractère analogue sur $\boldsymbol{M} p\left(X_{1}\right)$ (resp. $M p^{+}\left(X_{1}\right)$ ); il suffira donc de faire la démonstration pour $X=k$ ou pour $X=k^{2}$ suivant que la caractéristique de $k$ est autre que 2 ou égale à 2 .

Soit $\varphi$ un caractère de $M p(X)$ (resp. de $M p^{+}(X)$ ), continu ou non, qui coīncide avec $\theta$ sur T; $\varphi$ a la valeur 1 sur le groupe des commutateurs de $M p(X)$ (resp. $M p^{+}(X)$ ). Considérons le sous-groupe de $\boldsymbol{M}(X)$ (resp. de $M p^{+}(X)$ ) formé des éléments de la forme $\mathbf{d}(\alpha) \mathrm{t}(f)$, avec $\alpha \in \operatorname{Aut}(X), f \in Q(X)$; on vérifie aisément que son groupe des commutateurs comprend tous les éléments de la forme $\mathrm{t}(f)$; on a donc $\varphi(t(f))=1$ quel que soit $f \in Q(X)$. Posons $\varphi(\mathbf{d}(\alpha))=\lambda(\alpha)$ pour $\alpha \in \operatorname{Aut}(X)$, et $\varphi\left(\mathbf{d}^{\prime}(\gamma)\right)=\mu(\gamma)$ pour $\gamma \in \mathrm{Is}\left(X^{*}, X\right) ; \lambda$ est un caractère de Aut $(X)$, et la relation $d^{\prime}(\gamma \alpha)=d^{\prime}(\gamma) d(\alpha)$ donne $\mu(\gamma \alpha)=\mu(\gamma) \lambda(\alpha)$. Soit maintenant $f$ une forme quadratique non dégénérée sur $X$, associée à un morphisme $\varrho$ de $X$ sur $X^{*}$; compte tenu des différences de notations entre les Chapitres I et III, la relation (9) du Chapitre $1, \mathrm{n}^{\circ} 7$, donne :

$$
d^{\prime}\left(-\varrho^{-1}\right) t(f) d^{\prime}\left(\varrho^{-1}\right) t(f)=t(-f) d^{\prime}\left(-\varrho^{-1}\right)
$$

et la définition de $\gamma(f)$ au $\mathrm{n}^{\circ} 14$ de ce chapitre donne

$$
\mathbf{d}^{\prime}\left(-\varrho^{-1}\right) \mathbf{t}(f) \mathbf{d}^{\prime}\left(\varrho^{-1}\right) \mathbf{t}(f)=\gamma(f) \mathbf{t}(-f) \mathbf{d}^{\prime}\left(-\varrho^{-1}\right)
$$

En prenant la valeur de $\varphi$ pour les deux membres de cette dernière relation, on obtient

$$
\mu\left(\varrho^{-1}\right)=\gamma(f)
$$

Appliquons d'abord ce résultat au cas où $k$ n'est pas de caractéristique 2 et où $X=k$. Comme alors la forme $f$ associée à $\varrho$ est donnée par $f(x)=\varrho x^{2} / 2$, on obtient

$$
\gamma\left(\varrho x^{2} / 2\right)=\mu\left(\varrho^{-1}\right)=\mu(1) \lambda(\varrho)^{-1} .
$$


Au moyen de la proposition $3 \mathrm{du}$ Chapitre II, $\mathrm{n}^{\circ} 25$, on en conclut que $\gamma(f)$, pour une forme à $m$ variables $f(x)=\sum a_{i} x_{i}{ }^{2}$, dépend uniquement de $m$ et du discriminant $\prod a_{i}$ de $f$, donc que $\gamma(f)$ a même valeur pour toutes les formes à 4 variables de discriminant 1 , ce qui contredit la proposition $4 \mathrm{du}$ Chapitre II, $\mathrm{n}^{\circ} 28$. Considérons maintenant le cas $X=k^{2}$, avec $k$ de caractéristique 2 ; alors toute forme non dégénérée sur $X$ est équivalente à une forme $f_{1}(x, y)=a x^{2}+x y+b y^{2}$. Comme toutes les formes $f_{1}$ sont associées à un même morphisme (quels que soient $a, b$ ), la formule $\gamma(f)=\mu\left(\varrho^{-1}\right)$ montre que $\gamma$ prend la même valeur pour toutes les formes non dégénérées sur $X$. D'après la proposition $3 \mathrm{du}$ Chapitre II, $\mathrm{n}^{\circ} 25$, il s'ensuit que $\gamma$ prend la même valeur pour toutes les formes non dégénérées à 4 variables, ce qui est de nouveau en contradiction avec la proposition $4 \mathrm{du}$ même chapitre.

Il résulte de ce qui précède que les revêtements $S p_{2}(X), P s_{2}^{+}(X)$ définis au $n^{\circ} 43$ ne sont pas triviaux. Pour $k=\mathbf{R}$, l'existence d'un revêtement non trivial à deux feuillets du groupe symplectique est naturellement une conséquence du fait que celui-ci est alors un groupe de Lie connexe dont le groupe fondamental est $\mathbf{Z}$; quant à la représentation unitaire de ce revêtement qui est donnée par

$$
(\mathbf{S}, \Phi) \rightarrow \mathrm{S} \Phi \quad\left(\mathrm{S} \in S p_{2}(X), \Phi \in L^{2}(X)\right)
$$

ce n'est pas autre chose, dans ce cas, que celle qui a été récemment construite et étudiée par D. Shale [7]. Pour $k=C$, on sait que le groupe symplectique est simplement connexe, et on aurait pu déduire de là la trivialité de $M p(X)$ sur $S p(X)$. Il ne semble pas qu'on ait signalé jusqu'ici l'existence des revêtements $S p_{2}(X)$ et de leurs représentations unitaires $(\mathbf{S}, \Phi) \rightarrow \mathbf{S} \Phi$ dans les cas où $k$ est à valuation discrète.

45. Ce qui précède s'étend sans difficulté au cas adélique; comme nous n'aurons pas à faire usage de ces résultats, nous nous bornerons sur ce point à des indications sommaires.

Les notations étant celles des $n^{\text {os }} 37-38$, on va d'abord compléter par quelques remarques les résultats de ces numéros. Pour tout $v$, désignons par $\pi_{v}$ la projection canonique de $M p\left(X_{v}\right)$ sur $P s\left(X_{v}\right)=P s(X)_{v}$, par $\mathbf{T}_{v}$ le noyau de $\pi_{v}$, et par $\theta_{v}$ le caractère $\left(e_{v}, t\right) \rightarrow t$ de $\mathrm{T}_{v}, e_{v}$ étant l'élément neutre de $P s(X)_{v}$. Au $\mathrm{n}^{\circ} 38$, on a défini, pour $v \notin S_{0}$, le relèvement $\mathbf{r}_{v}^{\prime}$ de $P s(X)_{v}^{\circ}$ dans $M p\left(X_{v}\right)$; soit $M p(X)_{v}^{\circ}$ l'image de $P s(X)_{v}^{\circ}$ par $\mathbf{r}_{v}^{\prime}$; c'est un sous-groupe fermé de $M p\left(X_{v}\right)$. Pour tout $S \supset S_{0}$, posons

$$
M(S)=\prod_{v \in S} M p\left(X_{v}\right) \times \prod_{v \notin S} M p(X)_{v}^{\circ}
$$

D'après les $\mathrm{n}^{\text {os }} 37-38$, il y a, pour tout élément $\left(\mathbf{S}_{v}\right)$ de $M(S)$, un élément $\mathrm{S}$ de $M p(X)_{A}$ et un seul tel que l'on ait, pour toute fonction $\Phi \in L^{2}\left(X_{A}\right)$ de la forme (36) : 


$$
\mathbf{S} \Phi(x)=\prod_{v} \mathbf{S}_{v} \Phi_{v}\left(x_{v}\right)
$$

de plus, cette formule définit un homomorphisme ouvert $\left(\mathbf{S}_{v}\right) \rightarrow \mathbf{S}$ de $M(S)$ sur le sousgroupe ouvert

$$
M p(X)_{S}^{\circ}=\pi^{-1}\left(P s(X)_{S}^{\circ}\right)
$$

de $M p(X)_{A}$, homomorphisme dont le noyau est formé par les éléments $\left(t_{v}\right)$ de $M(S)$ pour lesquels $t_{v} \in \mathbf{T}_{v}$ quel que soit $v, \theta_{v}\left(t_{v}\right)=1$ pour $v \notin S$ (ce qui revient à dire que, pour $v \notin S, t_{v}$ est l'élément neutre de $\left.M p\left(X_{v}\right)\right)$, et $\Pi \theta_{v}\left(t_{v}\right)=1$.

D'après le $\mathbf{n}^{\circ} 20$ du Chapitre $\mathrm{I}, \mathbf{r}_{v}$ coïncide avec $\mathbf{r}_{v}^{\prime}$ sur l'ensemble $\Omega_{v}^{\circ}$ des éléments $s$ de $P s(X)_{v}^{\circ}$ tels que $\gamma(s)$ induise sur le réseau $\left(X^{*}\right)_{v}^{\circ}$ un isomorphisme de celui-ci sur $X_{v}^{\circ}$. De plus, si $\mathfrak{p}_{v}$ est l'idéal maximal de $\mathfrak{o}_{v}$, la réduction modulo $\mathfrak{p}_{v}$ détermine des homomorphismes de $X_{v}^{o}$ et $\left(X^{*}\right)_{v}^{o}$ sur un espace vectoriel $\mathfrak{X}_{v}$ sur le corps fini $\mathfrak{f}_{v}=\mathfrak{o}_{v} / \mathfrak{p}_{v}$, et sur le dual $\mathfrak{X}_{v}^{*}$ de $\mathfrak{X}_{v}$, respectivement; on en conclut que, pour presque tout $v$, la réduction modulo $\mathfrak{p}_{v}$ détermine un homomorphisme (et même, comme on le vérifie aisément, un homomorphisme surjectif) de $P s(X)_{v}^{\circ}$ dans $P s\left(\mathfrak{X}_{v}\right)$, qui applique $\Omega_{v}^{\circ}$ dans (et même sur) $\Omega\left(\mathfrak{X}_{v}\right)$.

Nous admettrons provisoirement que, pour tout espace vectoriel $\mathfrak{X}$ de dimension finie sur un corps $\mathfrak{l}, \Omega(\mathfrak{X})^{-1} \cdot \Omega(\mathfrak{X})$ est égal à $P s(\mathfrak{X})$ ou à $P s^{+}(\mathfrak{X})$ suivant que $\mathfrak{f}$ est de caractéristique autre que 2 ou égale à 2 ; ce point sera démontré au Chapitre $\mathrm{V}$ (corollaire 3 de la proposition $6, \mathrm{n}^{\circ} 47$ ). Supposons d'abord que $k$ ne soit pas de caractéristique 2; alors, pour presque tout $v$, on a

$$
\operatorname{Ps}\left(\mathfrak{X}_{v}\right)=\Omega\left(\mathfrak{X}_{v}\right)^{-1} \cdot \Omega\left(\mathfrak{X}_{v}\right) \text {, }
$$

d'où on conclut aisément que, pour presque tout $v, P s(X)_{v}^{\circ}=\left(\Omega_{v}^{\circ}\right)^{-1} \cdot \Omega_{v}^{\circ}$. D'autre part, pour chaque $v$, les résultats du $\mathrm{n}^{\circ} \mathbf{4 3}$ permettent de définir un caractère continu $\varphi_{v}$ de $M p\left(X_{v}\right)$ qui coïncide avec $\theta_{v}^{2}$ sur $\mathbf{T}_{v}$; comme on l'a vu, $\varphi_{v}$ est déterminé d'une manière unique par la condition que l'on ait, pour tout $s \in \Omega_{v}$ :

$$
\varphi_{v}\left(\mathbf{r}_{v}(s)\right)=(D(s) /-1)_{v} \gamma_{v}\left(q_{1}\right)^{2 m},
$$

où $m=\operatorname{dim}(X)$; lorsque -1 est un carré dans $k_{v}$, le second membre est égal à 1 pour tout $s \in \Omega_{v}$. Pour presque tout $v, k_{v}(\sqrt{-1})$ est égal, soit à $k_{v}$, soit à l'extension quadratique non ramifiée de $k_{v}$; dans l'un et l'autre cas, on a $(u /-1)_{v}=1$ pour toute unité $u$ de $\mathfrak{o}_{v}$; comme d'autre part on a $\gamma_{v}\left(q_{1}\right)=\mathrm{I}$ pour presque tout $v$ d'après le $\mathrm{n}^{\circ} 30$, il s'ensuit que, pour presque tout $v, \varphi_{v}$ a la valeur constante $\mathbf{l}$ sur $\mathbf{r}_{v}\left(\Omega_{v}^{\circ}\right)$. Mais pour presque tout $v$, comme on l'a vu, $\mathbf{r}_{v}$ coïncide avec $\mathbf{r}_{v}^{\prime}$ sur $\Omega_{v}^{\circ}$, et on a $P s(X)_{v}^{\circ}=\left(\Omega_{v}^{\circ}\right)^{-1} \cdot \Omega_{v}^{\circ}$; donc, pour presque tout $v, \varphi_{v}$ a la valeur constante 1 sur $M p(X)_{v}^{\circ}$. Il est clair alors que $\left(\mathbf{S}_{v}\right) \rightarrow \prod \varphi_{v}\left(\mathbf{S}_{v}\right)$ est un caractère de $M(S)$, égal à 1 sur le noyau de l'homomorphisme de $M(S)$ sur $M p(X)_{S}^{\circ}$ défini par (41), et que, par passage au quotient suivant ce noyau, puis à la limite inductive suivant $S$, on en déduit un caractère continu $\varphi_{A}$ de $M p(X)_{A}$, qui, sur $\mathbf{T}$, coïncide avec $(e, t) \rightarrow t^{2}$. Si on convient de dé- 
signer par $S p_{2}(X)_{A}$ le noyau de $\varphi_{A}$, on conclut de là, tout comme dans le cas local, que $M p(X)_{A}=S p_{2}(X)_{A} \cdot$ 'T et que $S p_{2}(X)_{A}$ est un " revêtement à deux feuillets " de $S p(X)_{A}$.

Montrons encore que $S p_{2}(X)_{A}$ contient $\mathbf{r}_{k}\left(P s(X)_{k}\right)$. Comme $P s(X)_{k}$ n'est autre que $\Omega(X)_{k}^{-1} \cdot \Omega(X)_{k}$, il suffira de montrer que $S p_{2}(X)_{A}$ contient $\mathbf{r}_{k}\left(\Omega(X)_{k}\right)$. Soit donc $s \in \Omega(X)_{k}$; on a alors $s \in \Omega_{v}$ pour tout $v$, et $s \in \Omega_{v}^{\circ}$ pour presque tout $v$; comme $\mathbf{r}_{v}$ coïncide avec $\mathbf{r}_{v}^{\prime}$ sur $\Omega_{v}^{\circ}$ pour presque tout $v$, il s'ensuit que $\left(\mathbf{r}_{v}(s)\right)$ est dans $M(S)$ pour $S$ assez grand. D'autre part, la formule (38) du $n^{\circ} 40$ donne ici

$$
\mathbf{r}_{k}(s) \Phi(x)=\int_{X_{A}^{*}} \Phi\left(x \alpha+x^{*} \gamma\right) \chi\left(f\left(x, x^{*}\right)\right) d x^{*}
$$

et la comparaison de cette formule avec (16) du $\mathrm{n}^{\circ} 13$ (qui, compte tenu des changements de notations, définit $\mathbf{r}_{v}$ pour tout $v$ ) montre que $\mathbf{r}_{k}(s)$ est l'image dans $M p(X)_{A}$ de l'élément $\left(\mathbf{r}_{v}(s)\right)$ de $M(S)$ par l'homomorphisme défini par (41). Dans ces conditions, pour que $\mathbf{r}_{k}(s)$ soit dans $S p_{2}(X)_{A}$, il faut et il suffit que l'on ait $\prod \varphi_{v}\left(\mathbf{r}_{v}(s)\right)=1$; or il en eșt évidemment ainsi si -1 est un carré dans $k$, et, dans le cas contraire, cela résulte immédiatement de la proposition 5 du $n^{\circ} 30$ et de ses conséquences énumérées au $n^{\circ} 30$ (c'est-à-dire de la loi de réciprocité quadratique).

Dans le cas où $k$ est de caractéristique 2, on procédera d'une manière analogue lorsque $m=\operatorname{dim}(X)$ est pair, en substituant naturellement la considération de $P_{s}+(X)$ à celle de $P s(X)$; si $m$ est impair et $\geqslant 3$, on plongera $X$ dans un espace $X^{\prime}$ de dimension $m+1$, comme dans le cas local. Le cas $m=1$ ne donne que des résultats triviaux.

\section{Compléments}

46. Comme aux $\mathrm{n}^{\mathrm{os}} 31-32$, on va d'abord considérer un espace vectoriel $X$ sur un corps quelconque $k$, et le groupe pseudosymplectique $P s(X)$ attaché à $X$. Pour $s=$

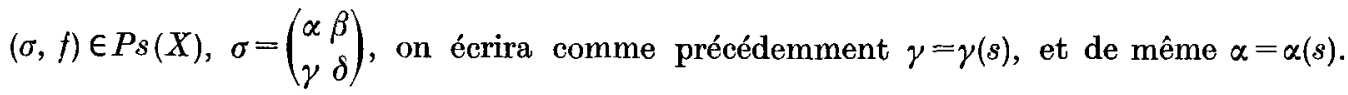
On conviendra de désigner par $P(X)$ le sous-groupe "parabolique " de $P s(X)$ formé des $s \in P s(X)$ pour lesquels $\gamma(s)=0$. Nous nous proposons d'écrire tout élément de $P s(X)$ sous une forme "normale " qui généralise l'expression (33) pour les éléments de $\Omega(X)$, et qui mette en évidence les classes à gauche dans $P s(X)$ suivant $P(X)$.

Soit d'abord $s=(\sigma, f) \in P(X)$. En exprimant au moyen de (32) que $s \in P s(X)$, et tenant compte de $\gamma(s)=0$, on obtient

$$
f\left(x_{2}, x_{1}^{*}+x_{2}^{*}\right)=f\left(0, x_{1}^{*}\right)+f\left(x_{2}, x_{2}^{*}\right) .
$$

En désignant par $g$ et $h$ les formes respectivement induites par $f$ sur $X$ et sur $X^{*}$, on en conclut que $h$ est additive et que l'on a 
A. WEIL

$$
f\left(x, x^{*}\right)=g(x)+h\left(x^{*}\right) .
$$

Il est immédiat alors que $t(g)^{-1} t^{\prime}(h)^{-1} s$ est de la forme $\left(\sigma^{\prime}, 0\right)$, donc, en vertu des remarques de la fin du $n^{\circ} 6$, de la forme $d(\lambda)$ avec $\lambda \in \operatorname{Aut}(X)$. On a donc

$$
s=t^{\prime}(h) t(g) d(\lambda)
$$

avec $h \in Q_{a}\left(X^{*}\right), g \in Q(X), \lambda \in \operatorname{Aut}(X)$; le calcul qu'on vient de faire montre même que $s$ se met sous cette forme d'une manière et d'une seule.

Soit maintenant $s=(\sigma, f)$ un élément quelconque de $P s(X)$; soit $\gamma=\gamma(s)$; soient $N$ le noyau de $\gamma, W$ un supplémentaire de $N$ dans $X^{*}$, et $Z=X^{*} \gamma$. Alors $\gamma$ induit sur $W$ un isomorphisme de $W$ sur $Z$, et il y a done une forme quadratique $f_{1}$ sur $Z$ telle que l'on ait $f(0, w)=f_{1}(w \gamma)$ pour tout $w \in W$; autrement dit, la formule

$$
f_{0}\left(x^{*}\right)=f\left(0, x^{*}\right)-f_{1}\left(x^{*} \gamma\right)
$$

définit une forme quadratique $f_{0}$ sur $X^{*}$ qui s'annule sur $W$. Mais d'autre part (32) donne, pour $n \in N, x^{*} \in X^{*}$ :

$$
f\left(0, n+x^{*}\right)=f(0, n)+f\left(0, x^{*}\right)
$$

ce qui implique en particulier que $f(0, n)$ est additive sur $N$. On conclut de là qu'on a $f_{0}(n+w)=f(0, n)$ pour $n \in N, w \in W$, ce qui montre que $f_{0}$ est une forme additive sur $X^{*}$.

47. On va déduire de là le résultat suivant :

Proposition 6. Soient $s=(\sigma, f), s^{\prime}=\left(\sigma^{\prime}, f^{\prime}\right)$ deux éléments de Ps $(X)$; soient $\gamma=\gamma(s)$, $\gamma^{\prime}=\gamma\left(s^{\prime}\right)$. Alors, pour que $s$ et $s^{\prime}$ appartiennent à une même classe à gauche suivant $P(X)$, il faut el il suffit qu'on ait $X^{*} \gamma=X^{*} \gamma^{\prime}$ et qu'il $y$ ait $f_{1} \in Q\left(X^{*} \gamma\right)$ telle que les deux formes

$$
x^{*} \rightarrow f\left(0, x^{*}\right)-f_{1}\left(x^{*} \gamma\right), \quad x^{*} \rightarrow f^{\prime}\left(0, x^{*}\right)-f_{1}\left(x^{*} \gamma^{\prime}\right)
$$

soient des formes additives sur $X^{*}$.

D'après le $n^{\circ} 46$, on peut en tout cas choisir $f_{1}$ de manière que la première de ces formes soit additive. Supposons alors qu'on ait $s^{\prime}=s^{\prime \prime} s$ avec $\gamma\left(s^{\prime \prime}\right)=0$; soient $s^{\prime \prime}=\left(\sigma^{\prime \prime}, f^{\prime \prime}\right)$, $\sigma^{\prime \prime}=\left(\begin{array}{ll}\alpha^{\prime \prime} & \beta^{\prime \prime} \\ 0 & \delta^{\prime \prime}\end{array}\right)$. Alors $\delta^{\prime \prime}$ est un automorphisme de $X^{*}$, et on a $\gamma^{\prime}=\delta^{\prime \prime} \gamma$, donc $X^{*} \gamma=X^{*} \gamma^{\prime}$. De plus, d'après le $n^{\circ} 46, f^{\prime \prime}\left(0, x^{*}\right)$ est une forme additive. Comme on a

on aura aussi

$$
f^{\prime}\left(0, x^{*}\right)=f^{\prime \prime}\left(0, x^{*}\right)+f\left(0, x^{*} \delta^{\prime \prime}\right),
$$

$$
f^{\prime}\left(0, x^{*}\right)-f_{1}\left(x^{*} \gamma^{\prime}\right)=f^{\prime \prime}\left(0, x^{*}\right)+f\left(0, x^{*} \delta^{\prime \prime}\right)-f_{1}\left(x^{*} \delta^{\prime \prime} \gamma\right),
$$


et le second membre est une forme additive en vertu de la définition de $f_{1}$. La condition de l'énoncé est done nécessaire. Réciproquement, supposons qu'elle soit satisfaite. Posons

$$
\sigma=\left(\begin{array}{ll}
\alpha & \beta \\
\gamma & \delta
\end{array}\right), \quad \sigma^{\prime}=\left(\begin{array}{ll}
\alpha^{\prime} & \beta^{\prime} \\
\gamma^{\prime} & \delta^{\prime}
\end{array}\right)
$$

et $s^{\prime \prime}=s^{\prime} s^{-1}$; on aura alors $\gamma\left(s^{\prime \prime}\right)=\gamma^{\prime} \delta^{*}-\delta^{\prime} \gamma^{*}$. Posons aussi $Z=X^{*} \gamma=X^{*} \gamma^{\prime}$, et soit $j$ l'injection de $Z$ dans $X$; on peut alors écrire $\gamma=\bar{\gamma} j, \gamma^{\prime}=\bar{\gamma}^{\prime} j$, où $\bar{\gamma}, \bar{\gamma}^{\prime}$ sont des morphismes de $X^{*}$ sur $Z$. Soit $\varrho_{1}$ le morphisme de $Z$ dans $Z^{*}$ associé à $f_{1}$; comme, en vertu de (32), le morphisme associé à $x^{*} \rightarrow f\left(0, x^{*}\right)$ est $\gamma \delta^{*}$, l'hypothèse faite sur $f\left(0, x^{*}\right)$ donne $\gamma \delta^{*}=\bar{\gamma} \varrho_{1} \bar{\gamma}^{*}$, ce qui s'écrit aussi

$$
\bar{\gamma}\left(j \delta^{*}-\varrho_{1} \bar{\gamma}^{*}\right)=\mathbf{0}
$$

d'où $j \delta^{*}=\varrho_{1} \bar{\gamma}^{*}$ puisque $\bar{\gamma}$ est surjectif. On en conclut $\delta j^{*}=\bar{\gamma} \varrho_{1}$; en remplaçant $f$ par $f^{\prime}$, on a donc aussi $\delta^{\prime} j^{*}=\bar{\gamma}^{\prime} \varrho_{1}$. Cela donne

$$
\bar{\gamma}^{\prime} \varrho_{1} \bar{\gamma}^{*}=\bar{\gamma}^{\prime} j \delta^{*}=\delta^{\prime} j^{*} \bar{\gamma}^{*}
$$

et par conséquent $\gamma^{\prime} \delta^{*}=\delta^{\prime} \gamma^{*}$, e'est-à-dire $\gamma\left(s^{\prime \prime}\right)=0$, ce qui achève la démonstration.

Il résulte en particulier de la proposition 6 que l'ensemble des classes à gauche de $P s(X)$ suivant $P(X)$ correspondant aux éléments $s$ de $P s(X)$ pour lesquels $\gamma(s)$ est de rang $r$ donné peut s'identifier avec un fibré vectoriel sur la grassmannienne des sous-espaces $Z$ de $X$ de dimension $r$, la fibre correspondant à un tel espace $Z$ étant l'espace vectoriel $Q(Z) / Q_{a}(Z)$.

Corollatre 1. Soit $s \in P s(X)$; soient $X_{1}$ l'image de $X^{*}$ par $\gamma(s), X_{2}$ un supplémentaire de $X_{1}$ dans $X$ et $\gamma_{1}$ un isomorphisme de $X_{1}^{*}$ sur $X_{1}$; soit aussi $Q_{1}^{\prime}$ un supplémentaire de $Q_{a}\left(X_{1}\right)$ dans $Q\left(X_{1}\right)$. Alors s se met d'une manière et d'une seule sous la forme

$$
s=t^{\prime}(h) t(g) d(\lambda)\left(d^{\prime}\left(\gamma_{1}\right) t\left(f_{1}\right) \otimes e_{2}\right)
$$

avec $h \in Q_{a}\left(X^{*}\right), g \in Q(X), \lambda \in \operatorname{Aut}(X), f_{1} \in Q_{1}^{\prime}, e_{2}$ désignant l'élément neutre de $P s\left(X_{2}\right)$.

Soit $\gamma=\gamma(s)$; il résulte du $\mathrm{n}^{\circ} 46$ (ou de l'énoncé même de la proposition 6 , si l'on y prend $s^{\prime}=s$ ) qu'il existe $f_{1} \in Q\left(X_{1}\right)$ telle que la forme

$$
x^{*} \rightarrow f\left(0, x^{*}\right)-f_{1}\left(x^{*} \gamma\right)
$$

soit additive. Comme cette condition détermine $f_{1}$ d'une manière unique à une forme additive près, on peut, d'une manière et d'une seule, y satisfaire en prenant $f_{1}$ dans $Q_{1}^{\prime}$. Soit alors $s^{\prime}=d^{\prime}\left(\gamma_{1}\right) t\left(f_{1}\right) \otimes e_{2}$; le critère de la proposition 6 montre immédiatement que $s$ et $s^{\prime}$ appartiennent à une même classe à gauche suivant $P(X)$. On peut done, d'une manière et 
d'une seule, mettre $s s^{\prime-1}$ sous la forme (42), ce qui donne bien pour $s$ l'expression (43). Réciproquement, si on suppose $s$ mis sous la forme (43) et qu'on définisse $s$ ' comme ci-dessus, on voit aussitôt, au moyen de la proposition 6 , que $f_{1}$ ne peut être autre que la forme qu'on a écrite plus haut; compte tenu de l'unicité de l'expression (42) pour tout élément de $P(X)$, cela achève la démonstration.

Coroltaire 2. Les hypothèses et notations étant les mêmes que dans le corollaire 1, s se met d'une manière et d'une seule sous la forme

$$
s=t(g) d(\lambda)\left(d^{\prime}\left(\gamma_{1}\right) t\left(g_{1}\right) \otimes t^{\prime}\left(h_{2}\right)\right)
$$

avec $g \in Q(X), \lambda \in \operatorname{Aut}(X), g_{1} \in Q\left(X_{1}\right), h_{2} \in Q_{a}\left(X_{2}^{*}\right)$

Procédant comme dans le corollaire 1, mais appliquant (42) à $s^{\prime} s^{-1}$, on obtient d'abord une expression

$$
s=d(\lambda)^{-1} t(g)^{-1} t^{\prime}(h)^{-1}\left(d^{\prime}\left(\gamma_{1}\right) t\left(f_{1}\right) \otimes e_{2}\right) .
$$

On peut écrire, pour $x^{*}=\left(x_{1}^{*}, x_{2}^{*}\right) \in X^{*}$ :

$$
-h\left(x^{*}\right)=h_{1}\left(x_{1}^{*}\right)+h_{2}\left(x_{2}^{*}\right)
$$

où $h_{1} \in Q_{a}\left(X_{1}^{*}\right), h_{2} \in Q_{a}\left(X_{2}^{*}\right)$; alors $t^{\prime}(h)^{-1}=t^{\prime}\left(h_{1}\right) \otimes t^{\prime}\left(h_{2}\right)$. Un calcul facile montre que $t^{\prime}\left(h_{1}\right) d^{\prime}\left(\gamma_{1}\right)$ s'écrit aussi $d^{\prime}\left(\gamma_{1}\right) t\left(h_{1}^{\prime}\right)$, avec $h_{1}^{\prime} \in Q_{a}\left(X_{1}\right)$. D'autre part il résulte immédiatement des identités du $n^{\circ} 32$ que $d(\lambda)^{-1} t(g)^{-1}$ peut s'écrire sous la forme $t\left(g^{\prime}\right) d\left(\lambda^{\prime}\right)$. En écrivant $g, \lambda, g_{1}$ au lieu de $g^{\prime}, \lambda^{\prime}, f_{1}+h_{1}^{\prime}$, on obtient (44). Quant à l'unicité, elle résulte de l'unicité de (43) et du calcul qu'on vient de faire, pris en sens inverse.

$\mathrm{Si}$, dans les corollaires 1 et 2 , on suppose $\gamma(s)=0$, ou, ce qui revient au même, $X_{1}=\{0\}$, $X_{2}=X$, on retrouve (42) ou une formule équivalente. D'autre part, le cas $s \in \Omega(X)$ équivaut à $X_{1}=X, X_{2}=\{0\}$; compte tenu des identités du $n^{\circ} 32$, l'application à ce cas du corollaire 2 donne à nouveau la formule (33). On va aussi déduire des corollaires ci-dessus un résultat qui était resté en suspens au Chapitre IV :

Corollatre 3. Soit $X$ un espace vectoriel sur un corps $k$; alors $\Omega(X)^{-1} \cdot \Omega(X)$ est égal à $P s(X)$ ou à $P_{s^{+}}(X)$ suivant que la caractéristique de $k$ est autre que 2 ou égale à 2.

Soient $s \in P s(X)$ et $s^{\prime} \in \Omega(X)$; mettons $s^{\prime}$ sous la forme (33) (ou, ce qui revient au même dans ce cas, sous la forme (44)) en écrivant $s^{\prime}=t(g) d^{\prime}(\gamma) t\left(g_{1}\right)$. Pour que l'on ait $s^{\prime} s \in \Omega(X)$, c'est-à-dire pour que $\gamma\left(s^{\prime} s\right)$ soit un isomorphisme de $X^{*}$ sur $X$, il faut et il suffit, comme le montre un calcul immédiat, que $\alpha\left(t\left(g_{1}\right) s\right)$ soit un automorphisme de $X$. Autrement dit, pour que $s$ appartienne à $\Omega(X)^{-1} \cdot \Omega(X)$, il faut et il suffit qu'il y ait $f \in Q(X)$ pour lequel $\alpha(t(f) s) \in \operatorname{Aut}(X)$. D'ailleurs, (33) montre que $\Omega(X)$ est une double classe suivant $P(X)$, ce qui est aussi évident directement; donc $\Omega(X)^{-1} \cdot \Omega(X)$ est une réunion de telles doubles 
classes. En mettant $s^{-1}$ sous la forme (43), et en posant $s_{1}=d^{\prime}\left(\gamma_{1}\right) t\left(f_{1}\right) \otimes e_{2}$, on voit donc que, pour que $s$ appartienne à $\Omega(X)^{-1} \cdot \Omega(X)$, il faut et il suffit que $s_{1}^{-1}$ y appartienne, ou encore qu'il y ait $f \in Q(X)$ tel que $\alpha\left(t(f) s_{1}^{-1}\right)$ soit un automorphisme de $X$. Or il est immédiat qu'on satisfait à cette condition en prenant pour $f$ une forme nulle sur $X_{2}$ et telle que $f-f_{1}$ soit non dégénérée sur $X_{1}$; un tel choix est toujours possible lorsque $k$ n'est pas de caractéristique 2 , et aussi lorsque $k$ est de caractéristique 2 et $X_{1}$ de dimension paire. Comme on a déjà observé au $n^{\circ} 32$ que cette dernière condition équivaut à $s \in P s^{+}(\mathrm{X})$, la conclusion s'ensuit. Notons en passant qu'on pourrait, dans le cas où $k$ est de caractéristique 2 , déduire de la proposition 6 et de ses corollaires les résultats rappelés sans démonstration à la fin du $n^{\circ} 32$ au sujet de $P s^{+}(X)$ et $P s^{-}(X)$.

48. Dans les chapitres précédents, on s'est souvent servi de la formule (33) du $\mathrm{n}^{\circ} 32$ (ou, ce qui revient au même, de la proposition $1 \mathrm{du} \mathrm{n}^{\circ} 7$ ) pour " relever " à $M p(X)$ des éléments de $\Omega(X)$; les corollaires 1 et 2 de la proposition 6 du $n^{\circ} 47$ permettent de faire de même pour un élément quelconque de $P s(X)$, lorsque $X$ est un espace vectoriel sur un corps local. On va appliquer cette remarque à la démonstration du résultat suivant :

Proposition 7. Soit $X$ un espace vectoriel sur un corps local $k$; soient $\mathbf{S} \in M p(X)$, $s=\pi(\mathbf{S})$ et $\gamma=\gamma(s)$. Soient $\xi, \xi^{\prime}$ deux automorphismes de $X$ tels que l'on ait $s d(\xi)=d\left(\xi^{\prime}\right) s$. Alors on a aussi $\mathbf{S d}(\xi)=\mathbf{d}\left(\xi^{\prime}\right) \mathbf{S}$ pourvu que l'on soit dans l'un des cas suivants : (i) on peut mettre s sous la forme (44) avec $h_{2}=0$; (ii) il y a un supplémentaire $X_{2}$ de $X_{1}=X^{*} \gamma$ dans $X$ qui est stable par $\xi$.

Comme $h_{2}$, dans (44), est une forme additive, on est toujours dans le cas (i) si $k$ n'est pas de caractéristique 2. Notons en passant que la condition $h_{2}=0$, qui en apparence dépend du choix de $X_{2}$ et de $\gamma_{1}$ dans le corollaire 2, est en fait équivalente à la suivante qui n'en dépend pas : $\left(i^{\prime}\right)$ si on écrit $s=(\sigma, f), f$ s'annule sur le noyau de $\gamma$.

En tout cas, $X_{2}$ et $\gamma_{1}$ ayant été choisis comme dans les corollaires 1 et 2 de la proposition 6 , on peut mettre $s$ sous la forme (44); il y a alors $a_{2} \in X_{2}$ tel que l'on ait, pour tout $x_{2}^{*} \in X_{2}^{*}$ :

$$
\chi\left(h_{2}\left(x_{2}^{*}\right)\right)=\chi\left(\left[a_{2}, x_{2}^{*}\right]\right) .
$$

D'autre part, la relation $s d(\xi)=d\left(\xi^{\prime}\right) s$ donne $\gamma \xi=\xi^{*-1} \gamma$, d'où $X_{1} \xi=X_{1}$, c'est-à-dire que $X_{1}$ est stable par $\xi$. En mettant $\xi$ sous forme matricielle par rapport à la décomposition $X=X_{1} \oplus X_{2}$ de $X$ en somme directe, on aura donc

$$
\xi=\left(\begin{array}{ll}
\xi_{1} & 0 \\
\eta & \xi_{2}
\end{array}\right)
$$

et (ii) revient à dire qu'il y a un choix de $X_{2}$ pour lequel $\eta=0$. 
Cela posé, $\mathbf{S}$ ne diffère que par un facteur scalaire de l'élément

$$
\mathbf{S}^{\prime}=\mathbf{t}(g) \mathbf{d}(\lambda)\left(\mathbf{d}^{\prime}\left(\gamma_{1}\right) \mathbf{t}\left(g_{1}\right) \otimes \mathbf{t}^{\prime}\left(h_{2}\right)\right)
$$

de $M p(X)$. D'autre part, l'hypothèse faite sur $s$ implique que $\operatorname{Sd}(\xi)$ et $\mathbf{d}\left(\xi^{\prime}\right) \mathrm{S}$ ne diffèrent que par un facteur scalaire $\theta \in T$; il s'ensuit que, pour tout $\Phi \in S(X)$, $\mathbf{S}^{\prime} \mathbf{d}(\xi) \Phi(0)$ et $d\left(\xi^{\prime}\right) S^{\prime} \Phi(0)$ ne diffèrent que par ce même facteur $\theta$. En explicitant les opérateurs qui interviennent dans la définition de $\mathbf{S}^{\prime}$, on voit que cela revient à dire qu'il existe $c>0$ tel que l'on ait

$$
\int \Phi\left(x_{1} \xi_{1}-a_{2} \eta,-a_{2} \xi_{2}\right) \chi\left(g_{1}\left(x_{1}\right)\right) d x_{1}=c \theta \int \Phi\left(x_{1},-a_{2}\right) \chi\left(g_{1}\left(x_{1}\right)\right) d x_{1}
$$

pour tout $\Phi \in S(X)$. Pour cela, il faut et il suffit évidemment que $a_{2} \xi_{2}=a_{2}$ et que l'on ait

$$
\chi\left(g_{1}\left(x_{1}\right)\right)=\theta \cdot \chi\left(g_{1}\left(x_{1} \xi_{1}-a_{2} \eta\right)\right)
$$

quel que soit $x_{1}$. Pour $x_{1}=0$, cela donne bien $\theta=1$ pourvu que $a_{2}=0$, ce qui a lieu dans le cas (i), ou $\eta=0$, ce qui a lieu dans le cas (ii).

COROLlaIRE. Soit $X$ un espace vectoriel sur un corps local $k$, et soit $G$ un sous-groupe de Aut $(X)$; supposons que $k$ ne soit pas de caractéristique 2 ou que $G$ soit complètement réductible. Soit $\mathbf{S}$ un élément de $M p(X)$ tel que $s=\pi(\mathbf{S})$ soit permutable avec $d(\xi)$ quel que soit $\xi \in G$; alors $\mathbf{S}$ est permutable avec $\mathbf{d}(\xi)$ quel que soit $\xi \in G$.

Si $k$ n'est pas de caractéristique 2, on n'a qu'à appliquer la proposition 7 , avec $\xi=\xi^{\prime}$. En tout cas, la démonstration de la proposition 7 montre que $X_{1}=X^{*} \gamma(s)$ est stable par $G$; si donc $G$ est complètement réductible, $X_{1}$ a un supplémentaire stable par $G$, et on est dans le cas (ii) de la même proposition.

49. On va maintenant définir des sous-groupes du groupe pseudosymplectique, qui joueront un grand rôle dans les applications à la théorie arithmétique des groupes classiques.

Soit $\mathcal{A}$ une algèbre sur un corps de base $k$; on la supposera toujours associative, de dimension finie sur $k$, et possédant un élément unité qu'on notera en général 1 ; on identifiera alors $k$ avec son image dans $\mathcal{A}$ au moyen de l'isomorphisme $t \rightarrow t \cdot 1$. On écrira éventuellement $\mathcal{A}_{k}$ au lieu de $\mathcal{A}$, particulièrement lorsqu'on voudra considérer le "cas adélique ".

On supposera de plus qu'on s'est donné sur $\mathcal{A}$ une involution $\iota$, c'est-à-dire un antiautomorphisme involutif de $\mathcal{A}$. considérée comme algèbre sur $k$; par définition, $\iota$ induit donc l'automorphisme identique sur $k$ lorsque $k$ est considéré comme sous-algèbre de $A$ comme il a été dit plus haut. 
On considérera des modules sur $\mathcal{A}$, dont on supposera toujours qu'ils sont de dimension finie sur $k$. La donnée de $\iota$ permet de considérer tout $\mathcal{A}$-module à droite $Y$ comme un $\mathcal{A}$-module à gauche, au moyen de la formule $t y=y t^{t} \quad(t \in \mathcal{A}, y \in Y)$. En particulier, soit $X$ un $\mathcal{A}$-module à gauche; son dual $\operatorname{Hom}_{A}\left(X, \mathcal{A}_{s}\right)$, c'est-à-dire l'espace des formes $\mathcal{A}$-linéaires sur $X$, est muni naturellement, comme il est bien connu, d'une structure de $\mathcal{A}$-module à droite; on conviendra de noter $X^{*}$ ce même dual muni d'une structure de $\mathcal{A}$-module à gauche de la manière qu'on vient d'expliquer. On notera $\left\{x, x^{*}\right\}$, pour $x \in X, x^{*} \in X^{*}$, la valeur en $x$ de la forme $\mathcal{A}$-linéaire sur $X$ qui correspond à $x^{*}$; on aura donc, par définition :

$$
\left\{t x, u x^{*}\right\}=t\left\{x, x^{*}\right\} u^{\imath} \quad\left(t \in \mathcal{A}, u \in \mathcal{A}, x \in X, x^{*} \in X^{*}\right),
$$

c'est-à-dire que $\left\{x, x^{*}\right\}$ est une forme sesquilinéaire sur $X \times X^{*}$.

Enfin, on supposera qu'on s'est donné aussi sur $\mathcal{A}$ une fonction trace $\tau$; on entendra par là une forme $k$-linéaire sur $\mathcal{A}$ (ou, pour être plus exact, sur l'espace vectoriel sur $k$ sous-jacent à $\mathcal{A}$ ), invariante par l'involution $\iota$ et telle que $(t, u) \rightarrow \tau(t u)$ soit une forme bilinéaire symétrique non dégénérée sur $\mathcal{A} \times \mathcal{A}$. Soit alors $X$ un $\mathcal{A}$-module à gauche; soit $f$ une forme $k$-linéaire sur $X$; alors, pour tout $x \in X, t \rightarrow f(t x)$ est une forme $k$-linéaire sur $\mathcal{A}$, et il y a donc un élément $F(x)$ de $\mathcal{A}$ et un seul tel que $f(t x)=\tau(t F(x))$ quel que soit $t$. Il est clair alors que $x \rightarrow F(x)$ est une forme $\mathcal{A}$-linéaire sur $X$ et qu'on a $f=\tau \circ F$. Par conséquent, la formule $f=\tau \circ F$ détermine une correspondance biunivoque entre les formes $k$-linéaires $f$ et les formes $\mathcal{A}$-linéaires $F$ sur $X$. En d'autres termes, on peut identifier $X^{*}$ avec le dual de l'espace vectoriel sur $k$ sous-jacent à $X$ au moyen de la formule

$$
\left[x, x^{*}\right]=\tau\left(\left\{x, x^{*}\right\}\right) \text {. }
$$

Comme on a convenu de ne considérer que des $\mathcal{A}$-modules de dimension finie sur $k$, il s'ensuit de plus qu'on peut identifier tout $\mathcal{A}$-module à gauche $X$ avec son bidual $\left(X^{*}\right)^{*}$ au moyen de la formule

$$
\left\{x, x^{*}\right\}=\left\{x^{*}, x\right\}^{\ell} .
$$

Soient encore $X$ et $Y$ deux $\mathcal{A}$-modules à gauche, et $\alpha$ un morphisme de $X$ dans $Y$, c'està-dire un élément de $\operatorname{Hom}_{A}(X, Y)$; alors le transposé $\alpha^{*}$ de $\alpha$ considéré comme morphisme d'espaces vectoriels sur $k$ appartient à $\operatorname{Hom}_{A}\left(Y^{*}, X^{*}\right)$, et l'on a

quels que soient $x \in X, y^{*} \in Y^{*}$.

$$
\left\{x \alpha, y^{*}\right\}=\left\{x, y^{*} \alpha^{*}\right\}
$$

Soient $X$ et $Y$ comme ci-dessus; alors, si $F$ est une forme sesquilinéaire sur $X \times Y$, il y a un morphisme $\alpha$ et un seul de $Y$ dans $X^{*}$ tel que l'on ait, quels que soient $x \in X, y \in Y$ :

$$
F(x, y)=\{x, y \alpha\}=\left\{y, x \alpha^{*}\right\}
$$

si $X=Y$, il faut et il suffit, pour que $F$ soit hermitienne, que l'on ait $\alpha=\alpha^{*}$.

$14-642946$ Acta mathematica. 111. Imprimé le 3 juin 1964. 
On dira qu'une forme quadratique $f$ sur l'espace vectoriel sur $k$ sous-jacent à $X$ est $\mathcal{A}$-quadratique si elle peut s'écrire $f(x)=\tau(F(x, x)$ ), où $F$ est une forme sesquilinéaire (non nécessairement hermitienne) sur $X \times X$; et on désignera par $Q(X / \mathcal{A})$ l'espace de ces formes. Il revient au même de dire que $Q(X / \mathcal{A})$ est l'ensemble des formes qui peuvent s'écrire

$$
f(x)=\tau(\{x, x \lambda\})
$$

où $\lambda$ est un morphisme (non nécessairement symétrique) de $X$ dans $X^{*}$; le morphisme associé à cette forme $f$ est alors $\varrho=\lambda+\lambda^{*}$. On notera $Q_{a}(X / \mathcal{A})$ l'espace des formes additives $\mathcal{A}$-quadratiques sur $X$. Si $k$ n'est pas de caractéristique 2 , et si $\varrho$ est le morphisme symétrique associé à une forme $f \in Q(X / \mathcal{A})$, la forme $F$ définie sur $X \times X$ par $F(x, y)=\{x, y \varrho / 2\}$ sera hermitienne et on aura $f(x)=\tau(F(x, x))$ pour tout $x$; cette dernière formule définit-donc alors une correspondance biunivoque entre $Q(X / \mathcal{A})$ et l'espace des formes hermitiennes sur $X \times X$.

Le groupe des automorphismes d'un $\mathcal{A}$-module $X$ sera noté $\operatorname{Aut}(X / \mathcal{A})$; de même, si $X$ et $Y$ sont des $\mathcal{A}$-modules, l'ensemble (éventuellement vide) des isomorphismes de $X$ sur $Y$ sera noté $\operatorname{Is}(X, Y / \mathcal{A})$. On notera $P s(X / k)$ le groupe pseudosymplectique attaché, conformément aux définitions du Chapitre III, à l'espace vectoriel sur $k$ sous-jacent à $X$; et on notera $P s(X / \mathcal{A})$ le sous-groupe de $P s(X / k)$ formé des éléments $(\sigma, f)$ de $P_{s}(X / k)$ pour lesquels $\sigma$ appartient à $\operatorname{Aut}\left(X \oplus X^{*} / \mathcal{A}\right)$ et $f$ à $Q\left(X \oplus X^{*} / \mathcal{A}\right)$. Avec les notations du Chapitre III, $\mathrm{n}^{\circ} 32, d(\alpha), d^{\prime}(\gamma), t(f), t^{\prime}\left(f^{\prime}\right)$ appartiendront à $P s(X / \mathcal{A})$ chaque fois que $\alpha, \gamma, f, f^{\prime}$ appartiennent respectivement à $\operatorname{Aut}(X / \mathcal{A}), \operatorname{Is}\left(X^{*}, X / \mathcal{A}\right), Q(X / \mathcal{A}), Q\left(X^{*} / \mathcal{A}\right)$. Les identités du $\mathrm{n}^{\circ} 32$ entre $d, d^{\prime}, t, t^{\prime}$ restent bien entendu valables ici.

50. Dans les applications qu'on fera des définitions du $n^{\circ} 49$, l'algèbre $\mathcal{A}$ sera le plus souvent supposée semisimple; lorsqu'il en est ainsi, tout sous-module d'un $\mathcal{A}$-module $X$ possède un supplémentaire. Si de plus $\mathcal{A}$ est simple, Is $\left(X^{*}, X / \mathcal{A}\right)$ n'est jamais vide, c'est-àdire que tout $A$-module à gauche $X$ est isomorphe à son dual, comme on le voit en considérant d'abord le cas où $X$ est un $\mathcal{A}$-module simple. Si $\mathcal{A}$ est " absolument semisimple " (c'est-à-dire telle que son extension à la clôture algébrique de $k$ soit semisimple) et est munie d'une involution $\iota$, il existe toujours sur $\mathcal{A}$ des fonctions traces $\tau$ au sens du $n^{\circ} 49$; on peut par exemple prendre pour $\tau$ la forme $k$-linéaire qui, sur chaque composante simple de $\mathcal{A}$, induit la " trace réduite 》.

Quand $\mathcal{A}$ n'est pas simple, on pourra faire usage du lemme suivant :

Lемм т. Soient $s \in P s(X / \mathcal{A}), \gamma=\gamma(s)$ et $Z=X^{*} \gamma$. Alors, pour que $Z$ admette un supplémentaire dans $X$, il faut et il suffit que le noyau $N$ de $\gamma$ en ait un dans $X^{*}$. Si do plus $\mathcal{A}$ est semisimple, les $\mathcal{A}$-modules $Z$ et $Z *$ sont isomorphes. 
Soit $Z_{*}$ l'orthogonal de $Z$ dans $X^{*}$; c'est le noyau de $\gamma^{*}$. De même, si $N_{*}$ est l'orthogonal de $N$ dans $X$, on a $N_{*}=X^{*} \gamma^{*}$. Posons $s=(\sigma, f)$ et $\sigma=\left(\begin{array}{l}\alpha \beta \\ \gamma \delta\end{array}\right)$. Soit $U$ l'image de $\{0\} \times X^{*}$ (considéré comme sous-module de $X \times X^{*}$ ) par $\sigma$; $U$ peut se définir aussi comme l'image de $X^{*}$ par le morphisme $x^{*} \rightarrow\left(x^{*} \gamma, x^{*} \delta\right)$; comme $\sigma$ est un automorphisme de $X \times X^{*}$, ce morphisme détermine un isomorphisme $\theta$ de $X^{*}$ sur $U$. D'autre part, $U$ est aussi l'ensemble des éléments $u=\left(y, y^{*}\right)$ de $X \times X^{*}$ tels que $u \sigma^{-1} \in\{0\} \times X^{*}$, ou autrement dit tels que $y \delta^{*}-y^{*} \gamma^{*}=0$. Soit $V=U \cap\left(\{0\} \times X^{*}\right)$; comme $Z_{*}$ est le noyau de $\gamma^{*}$, on a $V=\{0\} \times Z_{*}$; en même temps, on a $V=\theta(N)$ et $N=\theta^{-1}(V)$. Supposons maintenant que $Z$ ait un supplémentaire $Z^{\prime}$ dans $X$; alors l'orthogonal $Z_{*}^{\prime}$ de $Z^{\prime}$ dans $X^{*}$ est supplémentaire de $Z_{*}$ dans $X^{*}$, et $X \times Z_{*}^{\prime}$ l'est de $V$ dans $X \times X^{*}$. Il s'ensuit que $U_{1}=U \cap\left(X \times Z_{*}^{\prime}\right)$ est supplémentaire de $V$ dans $U$, done que $\theta^{-1}\left(U_{1}\right)$ l'est de $N$ dans $X^{*}$. Appliquons maintenant à $s^{-1}$ ce qu'on vient de démontrer pour $s$; comme $\gamma\left(s^{-1}\right)=-\gamma^{*}$, il s'ensuit que, si $N_{*}$ a un supplémentaire dans $X, Z_{*}$ en a un dans $X^{*}$. Enfin, $\gamma$ détermine en tout cas, par passage au quotient, un isomorphisme de $X^{*} / N$ sur $Z$; d'autre part, on peut identifier $X^{*} / Z_{*}$ avec $Z^{*}$, et ce qui précède montre que $Z_{*}$ est isomorphe à $V$ et par suite à $N$. Si $\mathcal{A}$ est semisimple, le fait que $N$ et $Z_{*}$ sont isomorphes entraîne que $X^{*} \mid N$ et $X^{*} / Z_{*}$ le sont aussi (par exemple en vertu du théorème de Jordan-Hölder), d'où la dernière assertion du lemme.

51. On conviendra de noter $P(X / k), P(X / \mathcal{A})$ les sous-groupes de $P s(X / k), P s(X / \mathcal{A})$ formés respectivement des éléments $s$ de ces groupes pour lesquels on a $\gamma(s)=0$. Il résulte du $\mathrm{n}^{\circ} 46$ que tout élément de $P(X / k)$ se met d'une manière et d'une seule sous la forme (42); si $s \in P(X / \mathcal{A})$, le calcul du $\mathrm{n}^{\circ} 46$ montre immédiatement que $s$ se met sous la forme (42) avec $h \in Q_{a}\left(X^{*} / \mathcal{A}\right), g \in Q(X / \mathcal{A}), \lambda \in \operatorname{Aut}(X / \mathcal{A})$. De même, si $s$ est un élément quelconque de $P s(X / \mathcal{A})$, le calcul fait dans la seconde partie du $n^{\circ} 46$ reste valable, et montre que $f_{1}$ appartient à $Q(Z / \mathcal{A})$ chaque fois que $W$ est un $\mathcal{A}$-module supplémentaire du noyau $N$ de $\gamma(s)$. Si donc, dans la proposition 6 , on suppose que $s$ et $s^{\prime}$ appartiennent à $P s(X / \mathcal{A})$, on pourra y prendre $f_{1} \in Q\left(X^{*} \gamma / \mathcal{A}\right)$ chaque fois que le noyau de $\gamma$ admet un supplémentaire dans $X^{*}$, ou, ce qui revient au même d'après le lemme 7 , chaque fois que $X^{*} \gamma$ en admet un dans $X$. Des remarques analogues s'appliquent aux corollaires 1 et 2 de la proposition 6 ; en vue d'applications ultérieures, nous expliciterons le résultat qui se déduit ainsi du corollaire 2 :

Propositron 8. Soit $X$ un module à gauche sur une algèbre $\mathcal{A}$ munie d'une involution et d'une fonction trace. Soient $s \in P s(X / \mathcal{A}), \gamma=\gamma(s)$ et $X_{1}=X^{*} \gamma ;$ supposons que $X_{2}$ soit un supplémentaire de $X_{1}$ dans $X$, et $\gamma_{1}$ un isomorphisme de $X_{1}^{*}$ sur $X_{1}$. Alors on peut, d'une manière et d'une seule, écrire s sous la forme 


$$
s=t(g) d(\lambda)\left(d^{\prime}\left(\gamma_{1}\right) t\left(g_{1}\right) \otimes t^{\prime}\left(h_{2}\right)\right)
$$

avec $g \in Q(X / \mathcal{A}), \lambda \in \operatorname{Aut}(X / \mathcal{A}), g_{1} \in Q\left(X_{1} / \mathcal{A}\right), h_{2} \in Q_{a}\left(X_{2}^{*} / \mathcal{A}\right)$.

On notera que l'existence d'un supplémentaire $X_{2}$ de $X_{1}$, et (d'après le lemme 7) celle d'un isomorphisme $\gamma_{1}$ de $X_{1}^{*}$ sur $X_{1}$ sont assurées chaque fois que $\mathcal{A}$ est semisimple.

Si $k$ est un corps local, on désignera par $M p(X / k)$ le groupe métaplectique attaché à l'espace vectoriel sur $k$ sous-jacent à $X$, et par $M p(X / \mathcal{A})$ l'image réciproque de $P s(X / \mathcal{A})$ dans $M p(X / k)$ par la projection canonique de $M p(X / k)$ sur $P s(X / k)$. Dans le cas adélique, les notations $M p(X / k)_{A}, M p(X / A)_{A}$ se définissent de même. L'application de la proposition $7 \mathrm{du} \mathrm{n}^{\circ} 48$ donne alors les résultats suivants :

Proposition 9. Soit $\mathcal{A}$ une algèbre sur un corps local $k$, munie d'une involution $\iota$ et d'une fonction trace; supposons que $k$ ne soit pas de caractéristique 2 ou que A soit semisimple. Soit $X$ un A-module à gauche. Alors tout élément de $M p(X / \mathcal{A})$ est permutable avec tout élément de $M p(X / k)$ de la forme $\mathrm{d}\left(\xi_{a}\right)$, où $\xi_{a}$ est l'homothétie $x \rightarrow a x$ de $X$ déterminée par un élément a de A tel que $a \cdot a^{\imath}=1$.

Si $a$ est un élément inversible de $\mathcal{A}$, il résulte de la définition de la structure de $\mathcal{A}$ module à gauche de $X^{*}\left(\right.$ cf. $\left.\mathrm{n}^{\circ} 49\right)$ que $d\left(\xi_{a}\right)=(\sigma, 0)$, où $\sigma$ est l'automorphisme

$$
\left(x, x^{*}\right) \rightarrow\left(a x,\left(a^{l}\right)^{-1} x^{*}\right)
$$

de l'espace vectoriel sur $k$ sous-jacent à $X \oplus X^{*}$; pour $a \cdot a^{\imath}=1, \sigma$ est donc l'homothétie $z \rightarrow a z$ de $X \oplus X^{*}$; par définition de $P s(X / \mathcal{A})$, il s'ensuit que tout élément de $P s(X / \mathcal{A})$ est alors permutable avec $d\left(\xi_{a}\right)$. On obtient le résultat annoncé en appliquant le cas (i) de la proposition 7 si $k$ n'est pas de caractéristique 2 , et le cas (ii) si $\mathcal{A}$ est semisimple.

COROLlaIR E. Soit $A_{k}$ l'anneau des adèles attaché à un corps de nombres ou à un corps de fonctions $k$. Soit $\mathcal{A}_{k}$ une algèbre sur $k$, munie d'une involution Ł et d'une fonction trace; supposons que $k$ ne soit pas de caractéristique 2 ou que $\mathcal{A}_{k}$ soit semisimple. Soit $G_{k}$ le groupe des éléments a de $\mathcal{A}_{k}$ tels que $a \cdot a^{\imath}=1$, et soit $G_{A}$ le groupe adélique correspondant. Soit $X_{k}$ un $\mathcal{A}_{k}$-module à gauche, et soit $X_{A}=X_{k} \otimes A_{k}$. Alors, pour tout $\mathrm{S} \in M p(X / A)_{A}$ et tout $a \in G_{A}$, les opérateurs $\Phi \rightarrow \mathrm{S} \Phi$ et $\Phi(x) \rightarrow \Phi(a x)$ sur $L^{2}\left(X_{A}\right)$ sont permutables.

Il suffit en effet de faire voir qu'ils sont permutables lorsqu'on les applique aux fonctions $\Phi$ de la forme définie par (36) au $n^{\circ} 38$; mais alors on est ramené au cas local, c'est-à-dire à la proposition 9.

52. En manière de conclusion, on va maintenant énoncer le résultat principal qui sera démontré dans le mémoire suivant, en application de la théorie exposée ici. 
Soit $k$ un corps de nombres algébriques; soit $\mathcal{A}_{k}$ une algèbre simple sur $k$, munie d'une involution $\iota$; pour fixer les idées, on prendra pour fonction trace, sur $\mathcal{A}_{k}$, la trace réduite $\tau$. On peut supposer, sans diminuer la généralité, que $k$ est le sous-corps du centre de $\mathcal{A}_{k}$ formé des éléments de celui-ci qui sont invariants par $\iota$.

Soit $G_{k}$ le groupe des éléments $a$ de $\mathcal{A}_{k}$ tels que $a \cdot a^{\imath}=1$; soit $G_{A}$ le groupe adélique correspondant; soit $d_{1} a$ la mesure de Haar sur $G_{A}$, normée de façon que $G_{A} / G_{k}$ soit de mesure 1. Soit $X_{k}$ un module à gauche sur $A_{k}$; soient $X_{A}=X_{k} \otimes A_{k}$ et $\Phi \in S\left(X_{A}\right)$. La formule qu'on se propose de démontrer est alors

$$
\int_{G_{A} / G_{k}} \sum_{\xi \in X k} \Phi(a \xi) d_{1} a=\sum_{s} \mathbf{r}_{k}(s) \Phi(0),
$$

où la sommation du second membre est étendue à un système complet de représentants des classes à gauche suivant $P\left(X_{k} / \mathcal{A}_{k}\right)$ dans $P s\left(X_{k} / \mathcal{A}_{k}\right)$; les deux membres sont absolument convergents, et la formule est valable, chaque fois qu'on a

$$
\operatorname{dim}_{k}\left(X_{k}\right)>4 \operatorname{dim}_{k} Q\left(X_{k} / \mathcal{A}_{k}\right)
$$

\section{Bibliographie}

[1]. BRúhat, F., Distributions sur un groupe localement compact et applications à l'étude des représentations des groupes $p$-adiques. Bull. Soc. Math. France, 89 (1961), 43-75.

[2]. Cartier, P., Über einige Integralformeln in der Theorie der quadratischen Formen. A paraittre aux Math. Ann.

[3]. MaCkEY, G., Some remarks on symplectic automorphisms (à paraittre).

[4]. Schwartz, L., Théorie des Distributions, tome II. Hermann et $\mathrm{C}^{\mathrm{de}}$, Paris 1951.

[5]. Segal, I. E., Foundations of the theory of dynamical systems of infinitely many degrees of freedom (I). Mat.-Fys. Medd. Danske Vid. Selsk., 31, no. 12 (1959), 1-39.

[6]. - - Transforms for operators and symplectic automorphisms over a locally compact abelian group. A paraître aux Math. Scand.

[7]. Shale, D., Linear symmetries of free boson fields. Trans. Amer. Math. Soc., 103 (1962), $149-167$.

Reçu le 30 septembre 1963 